\title{
Structure of the RZZ complex and molecular basis of Spindly-driven corona assembly at human kinetochores
}

Tobias Raisch ${ }^{1, \dagger}$, Giuseppe Ciossani ${ }^{2, k, \dagger}$, Ennio d'Amico ${ }^{2, \dagger}$, Verena Cmentowski ${ }^{2, \dagger}$, Sara

Carmignani $^{2}$, Stefano Maffini ${ }^{2}$, Felipe Merino ${ }^{1} \wedge$, Sabine Wohlgemuth ${ }^{2}$, Ingrid R. Vetter ${ }^{2}$, Stefan Raunser,@ \& Andrea Musacchio ${ }^{2,3, @ ~}$

Department of Structural Biochemistry, Max Planck Institute of Molecular Physiology, Otto-

13 Hahn Straße 11, 44227 Dortmund, Germany

14 Department of Mechanistic Cell Biology, Max Planck Institute of Molecular Physiology, Otto-

15 Hahn Straße 11, 44227 Dortmund, Germany

16 Centre for Medical Biotechnology, Faculty of Biology, University Duisburg-Essen, Essen,

17 Germany

*Corresponding authors: andrea.musacchio@mpi-dortmund.mpg.de; stefan.raunser@mpi-

†These authors contributed equally

\&Present address: European Institute of Oncology, Via Adamello 16, 20100 Milan, Italy

^Present address: Max Planck Institute for Developmental Biology, Department of Protein

Keywords: kinetochore; centromere; cell division; mitosis; chromosome segregation; RZZ;

34 Short title: Structure of the RZZ complex 
36 In metazoans, $a \approx 1$ megadalton (MDa) super-complex comprising the Dynein-Dynactin adaptor Spindly and the ROD-Zwilch-ZW10 (RZZ) complex is the building block of a fibrous biopolymer, the kinetochore fibrous corona. The corona assembles on mitotic kinetochores to promote microtubule capture and spindle assembly checkpoint (SAC) signaling. We report here a high-resolution cryo-EM structure that captures the essential features of the RZZ complex, including a farnesyl binding site required for Spindly binding. Using a highly predictive in vitro assay, we demonstrate that the SAC kinase MPS1 is necessary and sufficient for corona assembly at supercritical concentrations of the RZZ-Spindly (RZZS) complex, and describe the molecular mechanism of phosphorylation-dependent filament nucleation. We identify several structural requirements for RZZS polymerization in rings and sheets. Finally, we identify determinants of kinetochore localization and corona assembly of Spindly. Our results describe a framework for the long-sought-for molecular basis of corona assembly on metazoan kinetochores.

\section{Introduction}

Kinetochores are multi-subunit macromolecular assemblies that promote the bi-orientation and segregation of chromosomes during cell division (Musacchio and Desai, 2017; Navarro and Cheeseman, 2021). They are multi-layered structures built on specialized chromatin loci named centromeres. The kinetochore's inner layer, named the constitutive centromere-associated network (CCAN) binds directly to the centromeric chromatin. The kinetochore's outer layer,

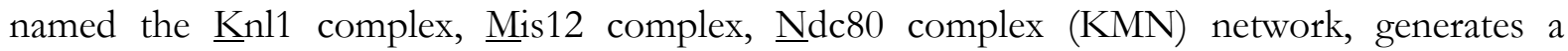
microtubule-binding interface. Additionally, the KMN network is a functional platform for the recruitment of several proteins that regulate the process of chromosome bi-orientation. Among the latter are proteins participating in the spindle assembly checkpoint (SAC), a cell cycle checkpoint that prevents mitotic exit before completion of bi-orientation (Lara-Gonzalez et al., 2021). SAC coordination is essential to prevent premature exit from mitosis or meiosis, which, by causing loss of chromosome cohesion in presence of unattached chromosomes, prevents successful chromosome partition to the daughter cells and is therefore essential for genome stability (Lara-Gonzalez et al., 2021). kinetochore layer named the kinetochore corona (Cooke et al., 1997; Hoffman et al., 2001; 
al., 1997). The corona is a fibrous crescent-shaped structure that is only formed transiently in prometaphase cells, before the achievement of end-on attachment of chromosomes to microtubules (Kops and Gassmann, 2020). In checkpoint arrested cells, for instance in cells treated with agents, such as nocodazole, that promote microtubule depolymerization, the corona assumes a very characteristic expanded crescent shape that surrounds the core kinetochore and often even fuses with the corona nucleated by the sister kinetochore (Hoffman et al., 2001; Magidson et al., 2015; Pereira et al., 2018; Sacristan et al., 2018; Wynne and Funabiki, 2015).

In recent years, there has been substantial progress on the investigation of the mechanisms of assembly and disassembly of the kinetochore corona, and of its contributions to microtubule binding and SAC regulation (Kops and Gassmann, 2020). Proteins whose recruitment to kinetochore has been associated with assembly of the corona include the 3-subunit ROD-ZwilchZW10 complex (named after the Drosophila melanogaster genes Rough Deal, Zwilch, and Zeste White 10, and abbreviated as RZZ), the microtubule plus-end directed motor CENP-E, the microtubulebinding protein CENP-F, a tight "core complex" of the SAC proteins MAD1 and MAD2, and the microtubule minus-end directed motor Dynein, in complex with its processivity factor Dynactin (Dynein-Dynactin will be abbreviated as DD) and with a DD adaptor named Spindly (Karess, 2005; Kops and Gassmann, 2020).

Microtubule motors in the corona facilitate the process of chromosome alignment at the metaphase plate. After initial microtubule capture, these motors coordinate minus- and plus-enddirected transport of chromosomes that promotes their alignment at the metaphase plate before conversion of kinetochore attachment from lateral (i.e. to the microtubule lattice) to end-on (i.e. into the kinetochore interface). This conversion engages the core microtubule receptor of the kinetochore, the NDC80 complex, a sub-complex of the KMN network. A crucial aspect of the lateral to end-on conversion is that it coincides with a sudden activation of DD and with the disassembly of the kinetochore corona, in a process traditionally known as "shedding" (Auckland et al., 2020; Basto et al., 2004; Howell et al., 2001; Mische et al., 2008; Sivaram et al., 2009; Varma et al., 2008; Williams et al., 1996; Wojcik et al., 2001).

Corona shedding also coincides with silencing of SAC signaling at the particular kinetochore undergoing conversion to end-on attachment (Kuhn and Dumont, 2017; Kuhn and Dumont, 2019). The corona promotes the SAC by providing a docking site for the recruitment of the MAD1:MAD2 core complex, which is crucially required for checkpoint signaling (De Antoni et al., 2005; Faesen et al., 2017). SAC silencing is therefore caused by the removal, during corona 
the checkpoint effector, the mitotic checkpoint complex (MCC) (Allan et al., 2020; Basto et al., 2000; Buffin et al., 2005; Caldas et al., 2015; Fava et al., 2011; Jackman et al., 2020; Kops et al., 2005; Matson and Stukenberg, 2014; Rodriguez-Rodriguez et al., 2018; Silio et al., 2015; Zhang et al., 2015).

The $812-\mathrm{kDa}$ RZZ complex, whose subunits are shown schematically in Figure 1A, is a 2:2:2 hexamer (Civril et al., 2010; Mosalaganti et al., 2017; Scaerou et al., 2001). The RZZ is considered the corona's building block (Mosalaganti et al., 2017; Pereira et al., 2018; Sacristan et al., 2018). While there is considerable interest in understanding how the RZZ promotes corona assembly, there is only limited structural insight into this process. An early structural analysis revealed the crystal structure of Zwilch and identified the 2209-residue ROD protein as a member of a family of proteins, which also includes clathrin, consisting of an $\mathrm{N}$-terminal $\beta$-propeller followed by a long $\alpha$-solenoid (Civril et al., 2010). Reconstitution of the RZZ and a single particle electron cryo microscopy (cryo-EM) structure, limited to an average resolution of approximately 10-12 offered the first comprehensive view of the organization of the RZZ hexamer (Altenfeld et al., 2015; Mosalaganti et al., 2017). The reconstruction demonstrated that two highly elongated ROD protomers are arranged in an anti-parallel configuration and that a ZW10 dimer cements this organization in its central region, while Zwilch occupies a more peripheral position, between ZW10 and the ROD $\beta$-propeller (Mosalaganti et al., 2017). Also based on homologous proteins of known structure but very limited sequence homology, structural models were built for ROD and ZW10 to fit the EM reconstruction (Mosalaganti et al., 2017). This previous work, however, failed to provide a detailed molecular description of the RZZ subunits and of their interactions. Here, we fill this gap by reporting a high-resolution structure of the RZZ complex, obtained by single-particle cryo-EM that finally reveals all its detailed structural features.

Spindly is a member of a large family of DD adaptors (Reck-Peterson et al., 2018) shown to activate dynein motility in vitro (Cianfrocco et al., 2015; Gama et al., 2017; Hoogenraad and Akhmanova, 2016; McKenney et al., 2014; Pereira et al., 2018; Sacristan et al., 2018; Schlager et al., 2014). How Spindly coordinates its interaction with RZZ with activation of DD motility and processivity remains poorly understood. Spindly binds directly to the RZZ complex through its C-terminal region (forming the complex abbreviated as RZZS), and engaging an RZZ module comprising the ROD $\beta$-propeller and Zwilch (Gama et al., 2017; Henen et al., 2021; Mosalaganti et al., 2017; Pereira et al., 2018; Sacristan et al., 2018). Furthermore, in humans and likely most other metazoans, Spindly is post-translationally modified on Cys602 with farnesyl, an isoprenoid lipid. This modification, which is required for the interaction of Spindly with RZZ, may engage a 
135 dedicated binding site on the ROD $\beta$-propeller (Gama et al., 2017; Holland et al., 2015;

136 Mosalaganti et al., 2017; Moudgil et al., 2015).

137 Besides interacting with RZZ, Spindly is also required for kinetochore recruitment of DD (Barisic 138 et al., 2010; Chan et al., 2009; Cheerambathur et al., 2013; Gama et al., 2017; Gassmann et al., 139 2008; Gassmann et al., 2010; Griffis et al., 2007; Raaijmakers et al., 2013; Starr et al., 1998; 140 Yamamoto et al., 2008). The determinants of RZZ binding and DD recruitment by Spindly are 141 separable. A region of Spindly, the Spindly motif, shown to be a conserved feature of adaptors, 142 can be mutated to abrogate kinetochore recruitment of DD (Gama et al., 2017; Gassmann et al., 143 2010; Pereira et al., 2018; Sacristan et al., 2018). The mutation is compatible with corona expansion 144 and chromosome bi-orientation, but preventing DD recruitment leads to a permanent SAC arrest 145 caused by the inability to disassemble (strip) the corona and silence the SAC (Gama et al., 2017; 146 Gassmann et al., 2010; Pereira et al., 2018; Sacristan et al., 2018).

147 Initial studies in humans and C. elegans identified conditions in vitro and in living cells for RZZ 148 assembly into filamentous structural mimics of the corona, pointing to the RZZ as a candidate 149 building block of the corona (Henen et al., 2021; Pereira et al., 2018; Sacristan et al., 2018). These 150 recent studies, however, also brought to light different minimal requirements for filament assembly 151 (Pereira et al., 2018; Sacristan et al., 2018), with species-specific differences and a persisting 152 question on whether Spindly is necessary for filament assembly and acts as gatekeeper in this 153 process. Corona assembly is limited to kinetochores and is sensitive to the cellular concentration of RZZ (Pereira et al., 2018), suggesting it requires a critical concentration that is exclusively reached upon RZZ recruitment to kinetochores in early prometaphase. Kinetochore recruitment, however, is not sufficient for corona assembly in human cells, because the depletion of Spindly or the inhibition of the SAC kinase MPS1 prevent expansion of the corona without preventing kinetochore recruitment of the RZZ(Pereira et al., 2018; Rodriguez-Rodriguez et al., 2018). Here, we have recapitulated with purified components in vitro the requirement for human ROD phosphorylation by MPS1 and Spindly binding for corona assembly. This assay allowed us to identify various additional requirements for nucleation of filaments by the RZZS complex, and to acquire structural information on the mechanism of filament assembly that was related to the highresolution structure of the RZZ. We present a model for corona assembly whose implications were extensively corroborated with experiments in mitotic cells. Collectively, our results greatly advance our understanding of a fundamental aspect of kinetochore structure and function. 
Reconstitution and structural analysis of the RZZ complex Using reconstituted human RZZ (Altenfeld et al., 2015; Mosalaganti et al., 2017), we previously reported a single particle cryo-EM reconstruction of the RZZ at an overall resolution of $\sim 10-12 \AA(1 \AA=0.1 \mathrm{~nm})$. As only the structure of Zwilch (Civril et al., 2010) (PDB ID 3IF8) had been experimentally determined, we had tried to account for the observed density by building ad hoc homology models of ROD or ZW10 and fitting them in the 3D reconstruction (Mosalaganti et al., 2017). Due to the very low resolution of the reconstructions, however, the resulting models were merely tentative.

To improve the resolution of the RZZ structure, we used an mCherry-tagged RZZ construct $\left({ }^{\mathrm{mCh}} \mathrm{RZZ}\right)$ that was better expressed than the previously used poly-histidine-tagged RZZ (see Methods). In addition, purified ${ }^{\mathrm{mCh}} \mathrm{RZZ}$ proved to be more stable than the previous construct, allowing us to determine the structure of the complex by cryo-EM at a resolution of $3.9 \AA$ (Figure 1B). The new reconstruction allowed us to build an essentially complete atomic model of ZW10 and the central region of ROD. In the periphery of RZZ, where the resolution of the reconstruction was lower than in the center and therefore did not allow unequivocal model building, we resorted to high-confidence AlphaFold2 (AF2; (Jumper et al., 2021; Tunyasuvunakool et al., 2021)) predictions, and used flexible fitting with minimal interventions, to fit them in the density (Figure 1 -Supplement 1).

While related to our previous low-resolution model in its general outline, the new model provides a detailed description of all crucial molecular features of the RZZ complex. The RZZ complex is a 2:2:2 hexamer with C2 symmetry. The 2-fold-related ROD chains (A and B) run in an antiparallel configuration that sets the $\sim 45-\mathrm{nm}$ long and $\sim 10 \mathrm{~nm}$ wide dimensions of the RZZ (Figure 1C-D). After an N-terminal 7-bladed $\beta$-propeller, the ROD chain transitions, near residue 395, into a short helical hairpin that begins an uninterrupted but irregular $\alpha$-solenoid that extends until the C-terminus.

Several proteins share with ROD a succession of an N-terminal $\beta$-propeller followed by a Cterminal $\alpha$-solenoid. These include Clathrin, COP1, the Nup155 and Nup145 nucleoporins, Sec31, Sec39, and even the APC1 subunit of the APC/C (Alfieri et al., 2017; Brohawn et al., 2008; Fath et al., 2007; Fotin et al., 2004; Lee and Goldberg, 2010; Stagg et al., 2006; Stagg et al., 2007; ter Haar et al., 1998; Watson et al., 2019). In comparison to the 1675-residue Clathrin heavy chain or the 1944-residue APC1, the 2209-residue ROD $\alpha$-solenoid is significantly more elongated and straighter (Figure 2 - Supplement 1A). Packing of successive helical hairpins against each other with a slight right- or a left-handed rotation is a typical pattern of regular $\alpha$-solenoids. This pattern is also observed for blocks of successive helical hairpins in ROD, but there are points where the 
hairpins rather pack almost at a right angle, deflecting the polypeptide chain (Figure 2 -

Supplement 1A). There are at least three points where the ROD chain bends sharply, around residues 856 and 1060 in the central region, and around residue 1905 in the $\mathrm{C}$-terminal region. The latter kink generates a characteristic C-terminal "hook" that is perpendicular to the opposite ROD chain (Figure 2A and Figure 2 - Supplement 1A). This is a prominent interaction interface between ROD-A and ROD-B, as residues 1790-2125, which encompass part of the hook domain, form a cradle that interacts with residues 505-690 of the opposing ROD protomer, including residues 655-680, situated in an inter-helical loop (Figure 2B).

209 Zwilch abuts the ROD $\beta$-propeller nd forms a direct, extensive interface with only one of the 210 ROD protomers, with only a small contact with the hook domain of the second ROD molecule 211 (Figure 2C). Contacts of Zwilch with ROD terminate around residue 850 of ROD, where Zwilch 212 also contacts ZW10 in a small 3-way interface. As expected, the structure of Zwilch in the RZZ 213 complex is closely related to the crystal structure of Zwilch obtained in isolation (Civril et al., 214 2010), but adopts a more open conformation due to a reciprocal rotation of Zwilch's two domains, 215 presumably elicited by contacts within the complex (Figure 2 - Supplement 1B).

216 Finally, ZW10 adopts a highly curved, U-shaped conformation, a major determinant of which is 217 the sharp bending around residue 395, situated between the N- and C-terminal domains (Figure 218 2D and Figure 2 - Supplement 1C). ZW10-related domains are found in Ds11 and Tip20, subunits 219 of vesicle tethering complexes in S. cerevisiae. Like ZW10, they both consist of two roughly equally 220 sized helical domains (Tripathi et al., 2009), but are characterized by different inter-domain angles 221 (Figure 2 - Supplement 1). Indeed, the isolated ZW10 shows high flexibility between its two 222 domains (Mosalaganti et al., 2017).

223 In the RZZ, the interface between the A and B protomers of ZW10, which intersects the 2-fold symmetry axis of the RZZ complex, is relatively small (Figure 2D-F). Accordingly, AF2 does not predict any solitary ZW10 dimer in a conformation seen in the RZZ complex (unpublished results). ZW10 A and B, however, are stably set inside an "eye" between the two ROD chains (compare panels $\mathrm{A}$ and $\mathrm{E}$ in Figure 2), with which they form a very extensive interaction interface. Specifically, residues 850-980, 1050-1090, and 1135-1400 of ROD-A interact with ZW10-A, ZW10-B, and ZW10-A, respectively. The N-terminal regions of ZW10 are prominent features that emerge almost perpendicularly from the RZZ's long axis. Together with the C-terminal region of ZW10, they are among the best conserved sequence features of the RZZ complex (Figure 1E-F; alignments are provided in Figure 1 - Supplement 2). 
ROD's farnesyl binding site The interaction of Spindly with RZZ is direct and requires (Mosalaganti et al., 2017). This residue is located in proximity of a prominent feature of the ROD $\beta$-propeller, the insertion of an $\alpha$-helical hairpin (residue 168-190) between strands $\beta 3 \mathrm{C}$ and $\beta 3 \mathrm{D}$ (Figure 3B and Figure 1 - Supplement 2). The hairpin abuts against blade 2 of the propeller, partly bending it and increasing its separation from blade 3, and generating a deep, roughly cylindrical cavity between the two blades (Figure 3C). Remarkably, Leu120 lines the entry point of the cavity. AF2 predicts the $\mathrm{C}$-terminal region of Spindly to interact with this region of the ROD $\beta$-propeller, and modelling the farnesyl group on Cys602 shows that the cavity is ideally dimensioned to receive the farnesyl group (Figure 3D-G).

The entire cavity is lined with hydrophobic residues, including Leu100, Leu108, Leu110, Leu119, Leu120, Phe124, Met153, Ile164, Leu167, Leu169, and Ile191, in addition to two polar residues, Asn122 and Ser193 (Figure 3E-F). To test the role of this pocket in the binding of farnesylated Spindly $\left(\right.$ Spindly ${ }^{\mathrm{F}}$ ), we tried to occlude it by generating two mutant RZZ complexes in which hydrophobic residues lining the farnesyl-binding pocket were replaced with bulkier ones (as described in the legend of Figure $3 \mathrm{G}$ ). Confirming our hypothesis, the resulting mutants were stable but apparently unable to interact with Spindly $^{\mathrm{F}}$ in a size-exclusion chromatography experiment, contrarily to wild type RZZ, with which Spindly ${ }^{\mathrm{F}}$ formed a stoichiometric complex (Figure 3G).

The C. elegans and D. melanogaster Spindly sequences have no C-terminal cysteine for isoprenylation (Holland et al., 2015). Analysis of ROD sequences in these organisms demonstrates differences predicted to ablate the hydrophobic farnesyl-binding cavity observed in human ROD. Specifically, in both species, the first of the two $\alpha$-helices in the $\alpha$-helical hairpin insertion that contributes to the architecture of the farnesyl-binding cavity is shorter by 3-residues (Figure 1 - Supplement 2). (CeROD) precisely in the center of the cavity, obstructing it and making it inviable for farnesyl binding (Figure 3 - Supplement 1).

MPS1 nucleates RZZS fibers The requirements for corona expansion remain incompletely characterized. For instance, deletion of the ROD-1 $\beta$-propeller (residues 1-372) promotes ectopic filament formation in C. elegans embryos, while the equivalent deletion (residues 1-375) prevents 
expansion in human cells (Gama et al., 2017; Pereira et al., 2018). In vitro, a complex of CeZW10

(CZW-1) and CeROD with a deleted N-terminal propeller assembles a polymeric filamentous structure, whereas a full-length ternary complex is unable to polymerize (Pereira et al., 2018). Conversely, in vitro filament assembly of human RZZ, elicited by mild heating, requires Spindly ${ }^{\mathrm{F}}$ (Sacristan et al., 2018). Furthermore, an N-terminal deletion removing 65 residues of Spindly promotes ectopic filament assembly in human cells even when Spindly is not farnesylated, an otherwise necessary condition for RZZ:Spindly binding and corona assembly (Sacristan et al., 2018).

To shed light on the corona assembly mechanism, we set out to dissect it in vitro with purified components. Using a spinning disk confocal microscope, we monitored polymerization of fluorescent mCherry-tagged $\mathrm{RZZ}$ or $\mathrm{RZZS} \mathrm{S}^{\mathrm{F}}$ under various conditions after their purification to homogeneity and thorough dephosphorylation. At $20^{\circ} \mathrm{C}$, neither ${ }^{\mathrm{mCh}} \mathrm{RZZ}$ nor ${ }^{\mathrm{mCh}} \mathrm{RZZS}^{\mathrm{F}}$ (at a concentration of $4 \mu \mathrm{M}$ ) formed visible polymers (unpublished results and see below). Conversely, and in agreement with our previous observations (Sacristan et al., 2018), incubation of ${ }^{\mathrm{mCh}}{ }^{\mathrm{R} Z Z S^{\mathrm{F}}}$ for 1 hour at $30^{\circ} \mathrm{C}$ promoted formation of copious fibers (Figure $4 \mathrm{~A}$ ). No fibers were observed with ${ }^{\mathrm{mCh}} \mathrm{RZZ}$ or ${ }^{\mathrm{mCh}}$ Spindly ${ }^{\mathrm{F}}$ (Figure $4 \mathrm{~A}$ ), indicating that Spindly ${ }^{\mathrm{F}}$ is required for fiber formation.

Previous studies identified a role of the MPS1 kinase, a central SAC component, in corona expansion in human cells (Rodriguez-Rodriguez et al., 2018; Sacristan et al., 2018). Regulatory effects of phosphorylation of corona components by additional mitotic kinases have also been reported (Allan et al., 2020; Barbosa et al., 2020; Pereira et al., 2018; Rodriguez-Rodriguez et al., 2018; Sacristan et al., 2018). We therefore asked if mitotic kinases influenced corona assembly in our assay in vitro. When subjected to sub-stoichiometric concentrations (typically 1/4 kinase/substrate ratio) of various mitotic kinases in vitro at $20^{\circ} \mathrm{C}$ in presence of ATP, ${ }^{\mathrm{mCh}} \mathrm{RZZS}^{\mathrm{F}}$ sample was phosphorylated by MPS1 on ROD, and by CDK1:Cyclin-B and to a minor extent by BUB1 on Spindly ${ }^{\mathrm{F}}$ (as visualized by staining with the Pro- ${ }^{\mathrm{TM}}$ Diamond Gel Staining Reagent;

\section{Figure 4B).}

293 In line with a role of MPS1 phosphorylation of ROD in corona assembly (Rodriguez-Rodriguez 294 et al., 2018; Sacristan et al., 2018), addition of MPS1 and ATP promoted spontaneous assembly of 295 fibers at $20^{\circ} \mathrm{C}$, an effect that was eliminated in presence of reversine, an MPS1 inhibitor 296 (Santaguida et al., 2010). Conversely, PLK1, Aurora B, BUB1, CDK1:Cyclin-B did not promote 297 fiber assembly, even when combined (Figure 4C). Subjecting ${ }^{\mathrm{mCh}} \mathrm{RZZ}$ or Spindly ${ }^{\mathrm{F}}$ to MPS1 298 phosphorylation before mixing them to form a complex showed that only ROD needs to be 299 phosphorylated for fibers to assemble (Figure 4 - Supplement 1A-B). Spindly required 
300 farnesylation for filamentation because a C602A mutant failed to filament (Figure 4 - Supplement

$301 \quad 1 C)$.

302 To corroborate these results in an in vivo setting, we released HeLa cells from a G2-phase arrest into mitosis in presence of nocodazole and reversine to depolymerize microtubules and inhibit MPS1, respectively. MPS1 inhibition by reversine was confirmed by severe reduction of BUB1 kinetochore levels (Figure 4 - Supplement 1D-E), as reported previously (Santaguida et al., 2010). Both Zwilch and Spindly decorated kinetochores when MPS1 was inhibited, albeit at a slightly reduced level in comparison with the control condition in absence of MPS1 inhibitors (Figure 4DE, quantified in panel F). However, corona expansion had been clearly completely inhibited in presence of reversine, because Zwilch and Spindly showed the same dotted appearance of inner

310 kinetochore markers instead of the crescent-like appearance observed in control cells (Figure 4D311 E).

312 Next, we asked if Spindly is necessary for corona expansion as predicted by our results in vitro.

313 After depleting Spindly by RNAi (Figure 4G-H), we detected CENP-E and Zwilch at reduced but 314 still highly significant levels on kinetochores of nocodazole-arrested mitotic cells. As for MPS1 315 inhibition, however, also in this case we observed an essentially complete failure to expand the 316 corona (Figure 4I-J). Thus, neither Spindly nor MPS1 phosphorylation are strictly required for 317 RZZ recruitment to the kinetochore. However, both are indispensable for expanding the corona.

318 Thus, the in vitro corona assembly assay we describe is an excellent predictor of cellular events at 319 the corona, and leads to conclude that the presence of RZZ, Spindly, and active MPS1 are 320 necessary to promote assembly of the corona at the kinetochore. Because corona assembly is 321 limited to kinetochores, however, there must be additional signals to enrich the corona 322 constituents to these subcellular compartments. When added to mitotic cells with an already 323 formed corona, the selective cyclin-dependent kinase 1 (CDK1) inhibitor RO3306 (Vassilev et al., 324 2006) promoted corona detachment from the kinetochore, but no corona disassembly, as 325 described previously (Pereira et al., 2018; Sacristan et al., 2018) (Figure 4 -Supplement 1F-G). 326 Conversely, the selective Aurora B kinase inhibitor Hesperadin (Hauf et al., 2003) erased the 327 recruitment of both RZZ (and therefore Spindly) and CENP-E to the kinetochore (Figure 4 328 Supplement $1 \mathrm{H})$. Thus, both CDK1 and Aurora B are essential for directing or retaining the 329 corona to the kinetochore, but our results in vitro strongly suggest that they do so on the 330 kinetochore side of the binding interface for the corona. 
The role of MPS1 phosphorylation Thr13 and Ser15 of ROD were previously identified as MPS1

333 substrates required for corona assembly (Rodriguez-Rodriguez et al., 2018). In agreement with 334 these results, we found that a ${ }^{\mathrm{mCh}} \mathrm{RZZS}^{\mathrm{F}}$ mutant complex where $\mathrm{ROD}^{\mathrm{Thr} 13}$ and $\mathrm{ROD}^{\mathrm{Ser} 15}$ were 335 mutated to alanine (T13A/S15A) did not assemble fibers (Figure 5A). The phosphomimetic 336 mutant T13E/S15E, on the other hand, allowed ${ }^{\mathrm{mCh}} \mathrm{RZZS}^{\mathrm{F}}$ to form fibers at $20^{\circ} \mathrm{C}$ and in presence 337 of reversine to abrogate MPS1 activity (Figure 5A and Figure 5 - Supplement 1A-B). The 338 T13E/S15E mutant, however, failed to form fibers when Spindly ${ }^{\mathrm{F}}$ was omitted. This confirms 339 that Spindly ${ }^{\mathrm{F}}$ is absolutely necessary for fiber formation even after bypassing MPS1 activity. 340 Collectively, these results confirm that our fiber formation assay in vitro recapitulates crucial aspects 341 of corona assembly.

342 The inability of the T13A/S15A mutant of ${ }^{\mathrm{mCh}} \mathrm{RZZS}^{\mathrm{F}}$ to form fibers did not reflect a requirement 343 of these residues in fiber assembly, because fibers of this mutant were observed after mildly heating 344 the sample to $30^{\circ} \mathrm{C}$ (Figure 5 - supplement 1A). Fibers never formed when Spindly ${ }^{\mathrm{F}}$ was omitted 345 (Figure 5 - supplement 1B). Thus, T13 and S15 may not be required for a direct physical 346 interaction of ${ }^{\mathrm{mCh}} \mathrm{RZZS}^{\mathrm{F}}$ complexes (protomers) in the fiber. Rather they may be required for auto347 inhibition of filament nucleation and growth. To shed light on how this region of ROD controls 348 fiber formation, we asked if we could structurally model it. The ROD $\beta$-propeller begins with the $349 \beta 7 \mathrm{~d}$ strand around residue Leu30 (Figure 5B-C). The N-terminal residues that precede this point 350 of entry into the $\beta$-propeller cannot be mapped with certainty in the reconstruction, due to its 351 limited resolution in this peripheral region. However, AF2 predicts that this region first augments 352 blade 7 of the ROD $\beta$-propeller with an additional $\beta$-strand $(\beta 7 \mathrm{e})$, external to the outermost $\beta 7 \mathrm{~d}$ 353 of the 4-strand propeller; then, after forming a helical turn that packs against the top part of blade 3547 , it also augments blade 6 with a short $\beta 6$ e strand that pairs with $\beta 6 \mathrm{~d}$, before finally entering the $355 \quad \beta$-propeller (Figure 5C).

356 Based on these observations, we speculate that phosphorylation of Thr13 and Ser15 by MPS1 357 restructures the $\mathrm{N}$-terminal region of ROD, relieving auto-inhibition and allowing interactions 358 required for the nucleation and growth of RZZS filaments, possibly mediated by blades 6 and 7 . 359 To test this idea, we reasoned that if MPS1 phosphorylation of ROD were exclusively required to 360 promote nucleation of ${ }^{\mathrm{mCh}} \mathrm{RZZS}^{\mathrm{F}}$ filaments, the stability of already formed filament should remain 361 unaffected after ROD dephosphorylation. Indeed, successful dephosphorylation of ${ }^{\mathrm{mCh}} \mathrm{RZZS}^{\mathrm{F}}$ 362 filaments with lambda phosphatase (Figure 5D) did not visibly interfere with filament number or 363 stability (Figure 5E). An ${ }^{\mathrm{mCh}} \mathrm{RZZS}^{\mathrm{F}}$ complex containing a ${ }^{\mathrm{mCh}} \mathrm{ROD}$ deletion mutant lacking the N364 terminal 15 residues $\left({ }^{\mathrm{mCh}} \mathrm{ROD}^{\Delta 15}\right)$ was insensitive to MPS1 phosphorylation, but, like the 
365 T13A/S15A mutant, formed fibers when heat-treated (Figure 5F). Thus, ${ }^{\mathrm{mCh}}{ }^{\mathrm{ROD}}{ }^{\Delta 15}$ remains auto-

366 inhibited, probably due to the residual capping of blade 6 with the $\beta 6$ e strand, which we speculate

367 to be crucially required for fiber assembly.

369 Zwilch contributes directly to fiber assembly Previous studies implicated two highly conserved Zwilch residues, Glu422 and Asp426, in corona expansion in humans and nematodes (Gama et al., 2017; Pereira et al., 2018). To assess if these residues also have a direct effect on corona assembly in vitro, we engineered a ${ }^{\mathrm{mCh}} \mathrm{RZZS} \mathrm{S}^{\mathrm{F}}$ complex containing alanine mutants of these residues (E422A/D426A). The E422A/D426A mutant complex failed to form fibers, both upon MPS1 phosphorylation and upon heating (Figure 5G). This was not caused by an impairment of the interaction of RZZ with Spindly, as the latter was unperturbed (Figure 5 - Supplement 1C). Thus, Zwilch contributes directly to fiber assembly. The two conserved Zwilch residues are solvent exposed and are part of a continuous face of the RZZ complex that also comprises blade 6 of the ROD $\beta$-propeller and the $\mathrm{N}$-terminal region of ZW10 (Figure $5 \mathrm{H}$ ), suggestive of an extensive interaction interface for corona expansion.

The RZZS polymers To shed further light into the polymerization mechanism, we used negativestain EM to visualize the ${ }^{\mathrm{mCh}} \mathrm{RZZS}{ }^{\mathrm{F}}$ fibers obtained after mild heating or incubation with MPS1 kinase. Under either condition, the fibers appeared as flat sheets, consisting of somewhat irregular filaments packing side-a-side. The sheets co-existing with unpolymerized complexes and with small oligomers (Figure 6A). Essentially identical sheets were obtained with a complex containing untagged ROD $\left(\right.$ RZZS $^{\mathrm{F}}$, Figure 6 - Supplement 1A), or with complexes expressing the ROD mutants T13A/S15A, T13E/S15E, and $\Delta 15$ (Figure 6 - Supplement 1B). The sheets were not sufficiently ordered for a successful application of cryo-EM reconstruction methods.

Polymerization attempts with an RZZS complex where Spindly was also tagged with an N-terminal mCherry moiety ( ${ }^{\mathrm{mCh}} \mathrm{RZZ} /{ }^{\mathrm{mCh}} \mathrm{S}^{\mathrm{F}}$ ) prevented formation of fibers and rather promoted formation of complete rings, or of segments thereof of comparable curvature (Figure 6B), and regardless of whether initiated by heat or MPS1. Essentially identical figures were also observed with an equivalent complex containing GFP-tagged Spindly ( ${ }^{\mathrm{mCh}} \mathrm{RZZ} /{ }^{\mathrm{GFP}} \mathrm{S}^{\mathrm{F}}$; Figure 6 - Supplement $\left.1 \mathrm{C}\right)$, even with untagged ROD (RZZ/GFP ${ }^{\mathrm{F}}$; Figure 6C). Cleavage of the GFP moiety from the latter construct after polymerization into rings promoted the lateral association of the filamentous rings into bundled rings, with a texture that was considerably less dense than that of the sheets, 
suggesting that bundles of rings do not pack as tightly as bundles of filaments in the sheets (Figure $6 C)$.

Thus, N-terminal tagging of Spindly promotes the assembly of rings or curved filaments. The curvature of the rings, whose average diameter is approximately $0.65 \mu \mathrm{m}$ (Figure $6 \mathrm{D}$ ), is remarkably similar to the curvature of kinetochore crescents when the corona expands (Magidson et al., 2015). Two-dimensional (2D) class averages of short segments of the negatively stained samples comprising a few consecutive ring subunits revealed a substantial orientation preference that ultimately prevented the successful calculation of a 3D reconstruction (Figure 6 - Supplement 1D). Similar analyses on filaments at cryogenic temperatures suffered from the same extreme orientation preferences and were unsuitable for coherent reconstructions (unpublished data). Nonetheless, these analyses revealed that the rings appear to have a period of approximately half of the RZZ length $(\approx 23 \mathrm{~nm})$ and a width comparable to that of the RZZ $(\approx 11 \mathrm{~nm}$ ) (Figure $6-$ Supplement 1E), suggesting that they form through staggering of individual RZZS complexes.

411 The role of Spindly To shed light on how Spindly promotes corona assembly, we investigated the 412 corona assembly propensity of various Spindly deletion mutants, including Spindly ${ }^{250-C}$ and 413 Spindly ${ }^{354-C}$, where the CC1a/b segment of Spindly (250-C) or the CC1a/b and CC2 segments 414 (354-C) are deleted, respectively (Figure 3A). Both Spindly constructs, upon farnesylation, 415 interacted with the RZZ complex in size-exclusion chromatography experiments (Figure 7A). 416 Conversely, a further deletion construct, Spindly ${ }^{440-\mathrm{C}}$, was unable to interact with the RZZ complex.

417 While Spindly ${ }^{250-C}$ supported corona expansion in vitro upon MPS1 phosphorylation and mild 418 heating, indistinguishably from full-length Spindly, Spindly ${ }^{354-\mathrm{C}}$ did not support corona assembly 419 (Figure 7B and Figure 7 - Supplement 1A). Furthermore, while Spindly ${ }^{34-C}$ bound the RZZ in 420 vitro, it was unable to decorate kinetochores after introduction by electroporation into HeLa cells 421 depleted of endogenous Spindly (Figure 7C-D, quantified in panel E). As expected, therefore, 422 there was no expansion of the corona in these cells, as shown by the dot-like appearance of CENP-

423 E, contrasting its crescent-like appearance observed in control cells with a well-formed corona. 424 Spindly decorates kinetochores even when corona expansion is suppressed with an MPS1 inhibitor 425 (Figure 4E), indicating that its localization to kinetochores is not contingent on corona expansion. 426 Thus, failure of Spindly $y^{354-C}$ to reach kinetochores is unlikely to reflect its inability to assemble the 427 corona (Figure 7C). Rather, Spindly ${ }^{354-C}$ may bind RZZ with reduced affinity, or may be unable to 428 interact with one or more additional kinetochore receptors ultimately required to stabilize the 429 RZZS complex. 
430 Collectively, these results indicate that a segment of Spindly encompassing residues 250-353

431 contains a critical determinant of corona assembly and kinetochore recruitment, possibly distinct

432 or even overlapping. To shed further light on this question, we took advantage of our previous

433 observation that Spindly ${ }^{1-275}$ and Spindly ${ }^{306-C}$ are stable Spindly construct (Mosalaganti et al., 2017;

434 Sacristan et al., 2018) to build a new deletion mutant, Spindly ${ }^{\Delta 276-306}$. Spindly ${ }^{\Delta 276-306}$ bound robustly

435 to the RZZ complex in size-exclusion chromatography experiments (Figure 7 - Supplement 1B),

436 but was unable to support corona expansion in vitro (Figure 7B) and failed to reach the kinetochore

437 (Figure 7F, quantified in panel G; note that the very modest mCherry signal shown to overlap with

438 the centromere and inner kinetochore is a localization artifact of the mCherry tag). In conclusion,

439 these results identify a segment comprising 31 residues of Spindly (276-306) as a crucial

440 determinant of Spindly kinetochore localization and corona expansion.

\section{Discussion}

443 The high-resolution cryo-EM structure of the RZZ complex reported here crowns a succession 444 of studies that began with the biochemical reconstitution and bioinformatic analysis of RZZ 445 subunits, the determination of the crystal structure of Zwilch, and the report of a low-resolution 446 EM reconstruction of the RZZ (Altenfeld et al., 2015; Civril et al., 2010; Mosalaganti et al., 2017).

447 Our new structural analysis leveraged a pipeline that combined experimental structure 448 determination using cryo-EM, model building based on experimental 3D reconstructions, and the 449 enhanced prediction capabilities of AlphaFold2 (Jumper et al., 2021; Tunyasuvunakool et al., 450 2021), which were instrumental for model building in more peripheral regions of the 451 reconstruction where the local resolution did not allow de novo model building. Collectively, this 452 pipeline allowed us to reveal the structure of the RZZ complex at near-atomic resolution. The new 453 structure explains how intermolecular interactions of the subunits promote the assembly of the 454 RZZ complex; it also explains how RZZ interacts with the farnesyl moiety of Spindly; finally, it 455 sets the basis for understanding how RZZ assembles into supramolecular structures in the corona.

456 We refined an in vitro assay for corona reconstitution that allowed us to probe several aspects of 457 the polymerization reaction. First, we demonstrate that RZZS oligomerization in vitro into flat 458 sheets or rings is kinetically controlled, and can be induced either by raising temperature or by 459 addition of the MPS1 kinase. Under all tested conditions, RZZ polymerization in vitro required 460 Spindly ${ }^{\mathrm{F}}$, implicating it as a crucial building block of the corona. Two non-mutually exclusive 461 possibilities are that Spindly ${ }^{\mathrm{F}}$ contributes directly to binding interfaces required for polymerization, 
efforts to reveal the structure of the RZZS ${ }^{\mathrm{F}}$ filament were thwarted by the limited order of the

fibers we have obtained and by a very limited number of orientations on the EM grids. Future work will have to address this bottleneck, shedding light on the organization of the individual RZZS complex and of its polymeric form.

467 Nonetheless, our studies identified and mutationally probed several crucial interfaces for 468 polymerization, including the $\mathrm{N}$-terminal region of Spindly and a conserved acidic residue pair in 469 Zwilch. Human RZZS ${ }^{\mathrm{F}}$ polymerizes efficiently at $30^{\circ} \mathrm{C}$ in vitro in the absence of a kinetochore 470 support, whereas its polymerization in cells is seeded by the kinetochore and never extends far from it. While this may seem to suggest that other control mechanisms prevent RZZS oligomerization away from kinetochores, it should be considered that our experiments in vitro were carried out at RZZS ${ }^{\mathrm{F}}$ concentrations (usually $4 \mu \mathrm{M}$ ) likely to be approximately two orders of magnitude higher than those existing in cells, as inferred by the fact that most SAC components have concentrations comprised between 10 and $100 \mathrm{nM}$ (Simonetta et al., 2009). High concentration of building blocks likely facilitates polymerization, and indeed RZZS ${ }^{\mathrm{F}}$ filaments became sporadic or were not any longer observed at mid-nanomolar concentrations of RZZS ${ }^{\mathrm{F}}$ (Figure 7 - Supplement 1C). A second crucial factor likely explaining why RZZS filaments form only at kinetochores is that RZZS polymerization appears to be kinetically controlled, with MPS1

480 phosphorylation acting as catalyst to remove a steric blockade to oligomerization involving the 481 ROD N-terminal region. As kinetochores enrich MPS1 during mitosis, and MPS1 activity is 482 highest at these structures, albeit not limited to them (Kuijt et al., 2020), polymerization may 483 become naturally spatially limited to kinetochores.

484 This mechanism of corona assembly bears similarities to the process of coat assembly that drives intracellular trafficking of membranous organelles. In addition to evident structural similarities, most notably of ROD with Clathrin and COPs, which are also characterized by a succession of an $\mathrm{N}$-terminal $\beta$-propeller and a $\mathrm{C}$-terminal $\alpha$-solenoid, both processes are spatially and kinetically controlled so that they occur only at defined cellular locales and in presence of appropriate triggers 489 (Arakel and Schwappach, 2018; Sigismund et al., 2021). The high-affinity binding site that drives 490 RZZ recruitment to the kinetochore remains elusive, but appears to be confined within the KMN 491 network (Caldas et al., 2015; Chan et al., 2009; Miller et al., 2008; Pagliuca et al., 2009; Pereira et 492 al., 2018; Sundin et al., 2011; Varma et al., 2013).

493 At least two kinases, in addition to MPS1, are also required for assembly and/or retention of the corona, CDK1 and Aurora B. Acute inhibition of these kinases results respectively in the detachment of the assembled corona from the kinetochore (CDK1) and in the complete depletion 
496

497

498

499

500

501

502

503

504

505

506

507

508

509

510

511

512

513

514

515

516

517

518

519

520

521

522

523

524

525

526

527

528

529

of corona components at kinetochores (Aurora B). In experiments with purified kinases in vitro, we did not find prominent Aurora B phosphorylation sites on the RZZS ${ }^{\mathrm{F}}$ complex, suggesting that Aurora B does not controls corona assembly directly. Because Aurora B is critically required for MPS1 recruitment to kinetochores (Nijenhuis et al., 2013; Santaguida et al., 2010), we suspect that Aurora B inhibition blocks the essential function of MPS1 as promoter of corona assembly. On the other hand, CDK1 phosphorylates Spindly ${ }^{\mathrm{F}}$ efficiently in vitro, but without triggering filamentation. It is possible that the detachment of the corona after CDK1 inhibition reflects an essential role of CDK1 phosphorylation of Spindly in its kinetochore recruitment (e.g. by creating a phospho-dependent binding site or conformational change). In this case, CDK1 inhibition may be recapitulated by expression of non-phosphorylatable mutants of Spindly. Alternatively, CDK1 may contribute to the generation of a binding site for the RZZS on the kinetochore.

Previous observations identified RZZ and Spindly as being both necessary for corona assembly in human cells (Rodriguez-Rodriguez et al., 2018; Sacristan et al., 2018). Our results in vivo are consistent with this tenet, but are further supported by polymerization experiments in vitro that showed a nearly perfect correlation with corona assembly in living cells. This coincidence argues that the determinants of corona assembly, after excluding the unknown receptor site in the kinetochore, are entirely contained with the RZZS complex. Thus, the RZZS emerges from our study as being sufficient to assemble the corona. This conclusion also explains why depletion of additional corona components, including CENP-E, CENP-F, DD, and MAD1-MAD2, does not visibly disrupt corona assembly and RZZ kinetochore recruitment (Allan et al., 2020; Ciossani et al., 2018; Gassmann et al., 2010). These proteins, on the other hand, may retain residual kinetochore localization even after depletion of RZZS components. The MAD1-MAD2 complex, for instance, requires the RZZ complex for kinetochore localization, and will localize to the kinetochore even if the corona cannot expand due to Spindly depletion (Rodriguez-Rodriguez et al., 2018). In another example, CENP-E and CENP-F, in addition to interacting with the corona, have also been shown to interact with the non-corona components BUBR1 and BUB1, respectively (Ciossani et al., 2018; Legal et al., 2020; Raaijmakers et al., 2018). We anticipate that our corona assembly assay may shed light on the mechanism of recruitment of these additional corona components.

A fundamental unresolved question in kinetochore biology is how the conversion of microtubule attachments from lateral to end-on promotes corona stripping. Plausibly, this sudden transition reflects a weakening of the interaction of the RZZS with its kinetochore receptor, leading to DDdirected detachment of the corona from the kinetochore. What triggers this change in binding affinity, however, remains unclear. Our studies, by unveiling the molecular features of the RZZ 
complex and by defining requirements for its physical interactions, provide an initial step towards the elucidation of this very complex and important question.

\section{Acknowledgments}

534 We are grateful to Oliver Hofnagel and Daniel Prumbaum for help in EM data collection, and to the Musacchio and Raunser laboratories for helpful discussion. This work was supported by the Max Planck Society (to A.M. and S.R.). A.M. acknowledges funding by the Marie-Curie Training Network DivIDE (project number 675737), European Research Council (ERC) through Synergy Research Foundation) through SFB1430 (Project-ID 424228829). Density maps of RZZ will be deposited into EMDB upon publication. Coordinates of the RZZ complex will be deposited to the PDB upon publication. The authors declare no competing financial interests.

\section{Author contributions (following CRediT model)}

544 Conceptualization: Andrea Musacchio

545 Data curation: N/A

546 Formal analysis: Tobias Raisch, Ingrid Vetter, Felipe Merino

547 Funding acquisition: Andrea Musacchio, Stefan Raunser

548 Investigation: Verena Cmentowski, Giuseppe Ciossani, Ennio d'Amico, Tobias Raisch

549 Project Administration: Andrea Musacchio, Stefan Raunser

550 Resources: Sara Carmignani, Stefano Maffini, Sabine Wohlgemuth

551 Supervision: Stefano Maffini, Andrea Musacchio, Stefan Raunser, Ingrid Vetter

552 Validation: Andrea Musacchio, Stefan Raunser, Ingrid Vetter

553 Visualization: Verena Cmentowski, Giuseppe Ciossani, Ennio d'Amico, Andrea Musacchio, 554 Tobias Raisch, Ingrid Vetter

555 Writing - original draft: Andrea Musacchio

556 Writing - review \& editing: Sara Carmignani, Giuseppe Ciossani, Verena Cmentowski, Ennio 557 d'Amico, Stefano Maffini, Felipe Merino, Andrea Musacchio, Tobias Raisch, Stefan Raunser, 558 Ingrid Vetter 


\section{$560 \quad$ Materials and Methods}

561 Protein expression and purification Expression and purification of Spindly and RZZ constructs were carried out essentially as previously described (Mosalaganti et al., 2017; Sacristan et al., 2018). Proteins were expressed using the BiGBac recombinant expression system (Weissmann et al., 2016). Bacmid was produced from EMBacY cells and used to transfect Sf9 cells to produce baculovirus. The baculovirus was subjected to three rounds of amplification, and used to infect TnaO38 cells. Cells were cultured for 72 hours before harvesting. A pellet from expression in 500 $\mathrm{ml}$ of TnaO38 cells was lysed by sonication in $100 \mathrm{ml}$ lysis buffer (50 mM HEPES pH 8.0, 250 $\mathrm{mM} \mathrm{NaCl}, 2 \mathrm{mM}$ TCEP, $50 \mathrm{mM}$ imidazole) supplemented with $1 \mathrm{mM}$ PMSF and protease inhibitor cocktail (Serva). The lysate was then cleared by centrifugation at $100000 \mathrm{~g}$ for 45 minutes. The cleared lysate was loaded onto a HisPrep Fast Flow column (Cytiva) pre-equilibrated in lysis buffer, and washed with $500 \mathrm{ml}$ lysis buffer, after which the bound protein was eluted with lysis buffer supplemented with $300 \mathrm{mM}$ imidazole. The eluate was diluted 1:5 in Buffer A $(50 \mathrm{mM}$ HEPES pH 8.0, $2 \mathrm{mM}$ TCEP), and applied to a $6 \mathrm{ml}$ Resource Q anion exchange column (Cytiva). The protein was then eluted using a $50 \mathrm{mM}$ to $500 \mathrm{mM} \mathrm{NaCl}$ gradient. Peak fractions were analyzed by SDS-PAGE, and those containing the relevant proteins were concentrated to $500 \mu \mathrm{l}$ volume, and subjected to size-exclusion chromatography on a Superdex S200 10/300 column (Cytiva) for Spindly, and on a Superose 6 10/300 for RZZ constructs, equilibrated in SEC buffer (50 mM HEPES pH 8.0, $250 \mathrm{mM} \mathrm{NaCl}, 2 \mathrm{mM}$ TCEP). Fractions were pooled and concentrated to $10 \mathrm{mg} / \mathrm{ml}$, snap-frozen, and stored at $-80{ }^{\circ} \mathrm{C}$ until use. For dephosphorylation, $4 \mu \mathrm{M}^{\mathrm{mCh}} \mathrm{RZZS} \mathrm{S}^{\mathrm{F}}$ complex or pre-formed MPS1-induced ${ }^{\mathrm{mCh}} \mathrm{RZZS}^{\mathrm{F}}$ polymers were incubated for 15 hours in Mbuffer at $20^{\circ} \mathrm{C}$ with or without $0.4 \mathrm{mg} / \mathrm{ml}$ Lambda phosphatase (produced in house), in presence of $10 \mu \mathrm{M}$ reversine.

583 Spindly ${ }^{\Delta 276-306}$ was expressed as an mCherry fusion in E. coli. BL21 CodonPlus cells were transformed with a pET28a plasmid containing the coding sequence for the mCherry tag, a PreScission cleavage sequence, and Spindly ${ }^{\Delta 276-306}$, and grown in $\mathrm{TB}$ at $37^{\circ} \mathrm{C}$ to an $\mathrm{OD}_{600}$ of 0.5 . Expression was induced with $0.4 \mathrm{mM}$ IPTG. The culture was then transferred into an incubator pre-cooled to $18^{\circ} \mathrm{C}$, and grown overnight before harvesting. The pellet was then snap-frozen and stored at $-80^{\circ} \mathrm{C}$ until purification. Untagged Spindly ${ }^{\Delta 276-306}$ was obtained by cleaving the mCherry tag with PreScission protease, by incubating $2 \mathrm{mg}$ of mCherry-Spindly ${ }^{\Delta 276-306}$ with $0.1 \mathrm{mg}$ of in- 
column equilibrated in SEC buffer to remove the tag and PreScission protease. Fractions were then pooled and concentrated to $10 \mathrm{mg} / \mathrm{ml}$, snap-frozen, and stored at $-80^{\circ} \mathrm{C}$ until use.

Production of MPS1 kinase An mCherry-MPS1-6His construct was generated by sub-cloning in

a pLIB vector. The corresponding baculovirus was generated in Sf9 insect cells (Wickham et al., 1992). After three rounds of amplification (V0, V1, and V2, 4 days each), $100 \mathrm{ml}$ of V2 were inoculated in 1 liter of Tnao38 cells. 24 hours after infection for expression, reversine (Santaguida et al., 2010) was added to the growth medium $(1 \mu \mathrm{M})$ to maximize expression yields. After 60 hours of expression at $27^{\circ} \mathrm{C}$, cells were pelleted, washed in PBS, pelleted again and either stored at $-80^{\circ} \mathrm{C}$ after flash-freezing in liquid nitrogen, or used directly for purification. Every purification step was performed on ice or at $4^{\circ} \mathrm{C}$. The pellet was resuspended in $\sim 100 \mathrm{ml}$ buffer A $(300 \mathrm{mM}$

$602 \mathrm{NaCl}, 50 \mathrm{mM}$ Hepes $\mathrm{pH}$ 8, 5\% Glycerol, $2 \mathrm{mM}$ TCEP, $10 \mathrm{mM}$ Imidazole $\mathrm{pH}$ 8) and supplemented with PMSF (1:100), protease-inhibitor mix HP Plus (1:500, Serva) and DNaseI (1:300, Roche), lysed by sonication and cleared by centrifugation at $108000 \mathrm{~g}$ for $45 \mathrm{~min}$. The cleared lysate was applied to $5 \mathrm{ml}$ Nickel-NTA (GE Healthcare) slurry beads previously equilibrated in buffer A and incubated on a rotating platform at $4^{\circ} \mathrm{C}$ for 2 hours. The supernatant was removed by centrifugation $\left(1500 \mathrm{~g}, 5 \mathrm{~min}, 4^{\circ} \mathrm{C}\right.$ ) and the beads were washed with $100 \mathrm{ml}$ buffer A. For the elution, the beads were incubated $\left(\sim 15 \mathrm{~min}\right.$ at $\left.4^{\circ} \mathrm{C}\right)$ in $15 \mathrm{ml}$ of buffer A supplemented with 300 $\mathrm{mM}$ Imidazole $\mathrm{pH}$ 8. Samples of the cleared lysate, of the supernatant, and of the elution were loaded on SDS-page for analysis. The $15 \mathrm{ml}$ elution was then concentrated, spun at max speed for

$61130 \mathrm{~min}$ in a bench-top centrifuge (at $4^{\circ} \mathrm{C}$ ) and finally loaded on a Superdex200 16/60 (GE 612 Healthcare) previously equilibrated in MPS1 buffer (250 mM NaCl, $50 \mathrm{mM}$ Hepes pH 8, 5\% 613 glycerol, $2 \mathrm{mM}$ TCEP). The protein was then concentrated, aliquoted and stored in $-80^{\circ} \mathrm{C}$ after 614 flash-freezing in liquid nitrogen.

616 In vitro farmesylation Farnesyltransferase $\alpha / \beta$ mutant (W102T/Y154T) was expressed and 617 purified as previously described (Mosalaganti et al., 2017). Spindly was diluted to $100 \mu \mathrm{M}$ in 618 farnesylation buffer (50 mM HEPES pH 8.0, $250 \mathrm{mM} \mathrm{NaCl}, 10 \mathrm{mM} \mathrm{MgCl}$, $2 \mathrm{mM}$ TCEP), and 619 farnesyltransferase was added to a final concentration of $30 \mu \mathrm{M}$. Farnesyl pyrophosphate (Sigma620 Aldrich) was added stepwise to a final concentration of $300 \mu \mathrm{M}$. The reaction mixture was 621 incubated at RT for 6 hours, after which it was centrifuged at 16,000 $\mathrm{g}$ for 10 minutes to remove 622 precipitate that formed during the reaction. The cleared reaction mixture was then loaded on a 623 Superose 6 column equilibrated in SEC buffer to remove the farnesyltransferase. The fractions 
containing Spindly were identified by SDS-PAGE and pooled, concentrated to a final concentration of around $5 \mathrm{mg} / \mathrm{ml}$, snap-frozen, and stored at $-80{ }^{\circ} \mathrm{C}$ until use.

Analytical size-exclusion chromatography Analytical gel filtration runs were performed on farnesylated Spindly, RZZ and Spindly were pre-incubated at a concentration of $2 \mu \mathrm{M}$ and $6 \mu \mathrm{M}$ respectively on ice for $1 \mathrm{~h}$ in SEC buffer. For runs with concurrent farnesylation, RZZ, Spindly, and FTase were incubated at a concentration of $5 \mu \mathrm{M}, 15 \mu \mathrm{M}$ and $7.5 \mu \mathrm{M}$ respectively for $2 \mathrm{~h}$ at room temperature in SEC buffer supplemented with $25 \mu \mathrm{M}$ FPP, followed by 30 min on ice.

Filamentation experiments with RZZS or RZZ complexes Heat-induced $R_{Z} Z \mathrm{~S}^{\mathrm{F}}$ filaments were prepared by incubating $4 \mu \mathrm{M}$ RZZ complex and $8 \mu \mathrm{M}$ Spindly ${ }^{\mathrm{F}}$ or $4 \mu \mathrm{M}$ preassembled RZZS ${ }^{\mathrm{F}}$ complex for $1 \mathrm{~h}$ at $30^{\circ} \mathrm{C}$ in $\mathrm{H}$-buffer $(50 \mathrm{mM}$ Hepes $\mathrm{pH}$ 7.5, $100 \mathrm{mM} \mathrm{NaCl}, 1 \mathrm{mM} \mathrm{MgCl}$ and 1 $\mathrm{mM}$ TCEP). Tags were removed from ${ }^{\mathrm{mCherry}} \mathrm{RZZ} \mathrm{GFP}^{\mathrm{GF}} \mathrm{S}^{\mathrm{F}}$ filaments by incubating the polymers for 1 hour at $30^{\circ} \mathrm{C}$ with $0.5 \mathrm{mg} / \mathrm{ml}$ Prescission protease (produced in house). MPS1-induced filaments were obtained by incubating $4 \mu \mathrm{M}$ RZZ complex and $8 \mu \mathrm{M}$ Spindly ${ }^{\mathrm{F}}$ or $4 \mu \mathrm{M}$ RZZS $^{\mathrm{F}}$ preassembled purified complex for 15 hours at $20^{\circ} \mathrm{C}$ in M-buffer $(50 \mathrm{mM}$ Hepes pH 7.5, $100 \mathrm{mM} \mathrm{NaCl}, 1 \mathrm{mM}$ $\mathrm{MgCl}_{2}$ and $1 \mathrm{mM}$ TCEP), supplemented with $2 \mathrm{mM}$ ATP at $\mathrm{pH} 8.0$ and in presence of $1 \mu \mathrm{M}$ MPS1. The effect of other mitotic kinases on $\mathrm{RZZS}^{\mathrm{F}}$ complex filamentation was tested in the same conditions using $1 \mu \mathrm{M}$ of purified protein kinase (produced in house). Reversine (Calbiochem) was dissolved at $10 \mathrm{mM}$ in DMSO and used at $10 \mu \mathrm{M}$ final concentration. Protein phosphorylation was monitored by ProQ Diamond phosphostaining (ThermoFisher Scientific) after SDS-PAGE separation. Independent MPS1 phosphorylation of RZZ and Spindly ${ }^{\mathrm{F}}$ protein stocks was carried out by incubating $8 \mu \mathrm{M} \mathrm{RZZ}$ and $16 \mu \mathrm{M}$ Spindly ${ }^{\mathrm{F}}$ overnight in M-buffer at $20^{\circ} \mathrm{C}$, supplemented with $2 \mathrm{mM}$ ATP pH 8.0 and $1 \mu \mathrm{M}$ MPS1.

Confocal imaging of RZZS $S^{F}$ filaments Glass flow chambers of about $10 \mu$ l volume were assembled using standard cover glasses and glass slides, held together by double-side tape (Teva). Heat- or MPS1-induced RZZS ${ }^{\mathrm{F}}$ filaments were diluted to $0.5 \mu \mathrm{M}$ in $\mathrm{H}$ - or $\mathrm{M}$ - buffer (see above), respectively, and imaged in the glass chambers, at room temperature using a spinning disk confocal device on the $3 \mathrm{i}$ Marianas system at 63X magnification. Sample images were acquired as 5-stacks of z-sections at $0.27 \mu \mathrm{m}$, converted into maximal intensity projections, exported and processed with Fiji (Schindelin et al., 2012). 
Cell culture, plasmid transfection, microinjections and imaging Cell culture and drug treatment:

HeLa and DLD-1 cells were grown in Dulbecco's Modified Eagle's Medium (DMEM; PAN

Biotech) supplemented with $10 \%$ tetracycline-free FBS (PAN Biotech), and L-glutamine (PAN hesperadin at $500 \mathrm{nM}$ (Merck), and reversine (Cayman Chem.) at $500 \mathrm{nM}$. Cell transfection and electroporation: Depletion of endogenous Spindly was achieved through reverse transfection with 50 nM Spindly siRNA (5'-GAAAGGGUCUCAAACUGAA-3' obtained from Sigma-Aldrich) for 48 hours with RNAiMAX (Invitrogen, Carlsbad, California, United States). For rescue experiments, 24 hours after Spindly depletion, we electroporated recombinant Spindly constructs, either unlabeled or labeled with an N-terminal mCherry, at a concentration of $7 \mu \mathrm{M}$ in the electroporation slurry (as previously described in Alex et al., 2019) (Neon Transfection System, Thermo Fisher). Control cells were electroporated with mCherry or electroporation buffer, respectively. Following an 8 hours recovery, cells were treated with $9 \mu \mathrm{M}$ RO3306 (Calbiochem) for 15 hours. Subsequently, cells were released into mitosis in presence of $3.3 \mu \mathrm{M}$ nocodazole (Sigma) for 1 hour. Immunofluorescence: Cells were grown on coverslips pre-coated with Poly-Llysine (Sigma-Aldrich). Cells were pre-permealized with 0.5\% Triton X-100 solution in PHEM (Pipes, HEPES, EGTA, $\mathrm{MgCl}_{2}$ ) buffer supplemented with $100 \mathrm{nM}$ Microcystin for 5 minutes before fixation with 4\% PFA in PHEM for 20 minutes. After blocking with 5\% boiled goat serum (BGS) in PHEM buffer, cells were incubated for 2 hours at room temperature with the following primary antibodies: BUB1 (mouse, Abcam, ab54893, 1:400), CENP-E (mouse, Abcam, ab5093, 1:200), Spindly (rabbit, Bethyl, A301-354A, 1:1000), Zwilch (rabbit, made in-house, SI520, 1:900), CREST/anti-centromere antibodies (Antibodies, Inc., 1:200) diluted in $2.5 \%$ BGS-PHEM supplemented with $0.1 \%$ Triton-X100. Subsequently, cells were incubated for 1 hour at room temperature with the following secondary antibodies: Goat anti-mouse Alexa Fluor 488 (Invitrogen A A11001), donkey anti-rabbit Rhodamine Red (Jackson Immuno Research 711-295152), donkey anti-rabbit Alexa Fluor 488 (Invitrogen A21206), goat anti-human Alexa Fluor 647

685 (Invitrogen, Carlsbad, California, United States). All washing steps were performed with PHEM-

686 T buffer. DNA was stained with $0.5 \mu \mathrm{g} / \mathrm{ml}$ DAPI (Serva) and Mowiol (Calbiochem) was used as mounting media. Cell imaging: Cells were imaged at room temperature using a spinning disk confocal device on the 3i Marianas system equipped with an Axio Observer Z1 microscope (Zeiss),

689 a CSU-X1 confocal scanner unit (Yokogawa Electric Corporation, Tokyo, Japan), $100 \times / 1.4 \mathrm{NA}$ 690 Oil Objectives (Zeiss), and Orca Flash 4.0 sCMOS Camera (Hamamatsu). Images were acquired 
691

692

693

694

695

696

697

698

699

700

701

702

703

704

705

706

707

708

709

710

711

712

713

714

715

716

717

718

719

720

721

722

723

as $z$ sections at $0.27 \mu \mathrm{m}$. Images were converted into maximal intensity projections, exported, and converted into 8-bit tiff files. Automatic quantification of single kinetochore signals was performed using the software Fiji with background subtraction. Measurements were exported in Excel (Microsoft) and graphed with GraphPad Prism 9.0 (GraphPad Software). The figures were arranged using Adobe Illustrator 2022 software.

Negative stain electron microscopy sample preparation and image analysis $4 \mu \mathrm{l}$ of $20-100 \mathrm{nM}$ RZZS $^{\mathrm{F}}$ filaments were deposited on freshly glow-discharged Formvar/Carbon (Quantifoil) film supported copper grid Cu400 (Sigma Aldrich) and incubated for $1 \mathrm{~min}$. Once removal of the excess of sample was blotted away with filter paper, the grids were washed two times with $10 \mu \mathrm{l}$ of H- or M-buffer (Heat- or Mps1-induced filaments, respectively), then once with $10 \mu \mathrm{l}$ of $0.75 \%$ (w/v) uranyl formate (Sigma Aldrich). After staining with $10 \mu \mathrm{l}$ of $0.75 \%$ (w/v) uranyl formate for $30 \mathrm{sec}$, grids were blotted, dried and visualized at $120 \mathrm{kV}$ using a Tecnai Spirit equipped with a $\mathrm{LaB}_{6}$ cathode and a $4000 \times 4000$ CMOS detector F416 (TVIPS). Images were recorded at a nominal magnification of 21-42,000x. Single measurements of the diameter of RZZS ${ }^{\mathrm{F}}$ circular polymers were performed by processing negative stain EM images with Fiji (NIH). Values were exported in Excel (Microsoft) and graphed with GraphPad Prism 6.0 (GraphPad Software). 2D classification of ${ }^{\mathrm{mCh}} \mathrm{RZZ} \mathrm{Z}^{\mathrm{GFP}} \mathrm{S}^{\mathrm{F}}$ filaments was performed using ISAC (Yang et al., 2012) within SPHIRE (Moriya et al., 2017). 148 images were collected at a magnification of 42000x resulting in 2.6 A/pix. Straight filament sections were manually selected, and segments of 256x256 px and an overlap of $115 \mathrm{px}$ were extracted from those, resulting in 2730 particles. Classification was performed with standard parameters, using a radius of $120 \mathrm{px}$ and a maximum of 50 members per class.

Cryo-EM grid preparation and data acquisition Grids were prepared using a Vitrobot Mark IV (Thermo Fisher Scientific) at $13{ }^{\circ} \mathrm{C}$ and $100 \%$ humidity. $4 \mu \mathrm{l}$ of RZZ at a concentration of 5 $\mathrm{mg} / \mathrm{ml}$ and supplemented with $0.02 \%$ Triton were applied to glow-discharged Quantifoil R2/1 grids and excess liquid removed by blotting ( 3.5 seconds at blot force -3$)$ before vitrification in liquid ethane. Cryo-EM data were acquired on a Titan Krios electron microscope (Thermo Fisher Scientific) equipped with a field emission gun. Two datasets with 1968 and 5794 movies, respectively, were recorded on a K3 camera (Gatan) operated in super-resolution mode at a nominal magnification of 105,000 , resulting in a super-resolution pixel size of $0.45 \AA$. A Bioquantum post-column energy filter (Gatan) was used for zero-loss filtration with an energy 
width of $15 \mathrm{eV}$. Total electron exposure of 59 and $60 \mathrm{e}-/$ A2, respectively, was distributed over 60 frames. Data were collected using the automated data collection software EPU (Thermo Fisher Scientific), with three exposures per hole and a set defocus range of -1.2 to $-2.7 \mu \mathrm{m}$. Details of data acquisition parameters can be found in Supplementary Table 1.

Cryo-EM data processing On-the-fly data pre-processing, including correction of beam-induced motion and dose-weighting by MotionCor2 (Zheng et al., 2017), CTF parameter estimation using CTFFIND4 in movie mode (Rohou and Grigorieff, 2015), and particle picking using a custom neural network in SPHIRE-crYOLO (Wagner et al., 2019), was performed within TranSPHIRE (Stabrin et al., 2020). 74,836 four-fold binned particles with dimensions of 180x180 pixels were extracted from the first dataset using SPHIRE (Moriya et al., 2017), and used for 2D classification in ISAC. An initial model was calculated in RVIPER using 81 good 2D classes and by imposing C2 symmetry. Initial 3D reconstruction in MERIDIEN was performed using 49,656 two-fold binned particles of the second dataset which were assigned to well-defined $2 \mathrm{D}$ classes and also with imposed C2 symmetry, resulting in a $4.7 \AA 3 \mathrm{D}$ reconstruction. Recentered particles from all micrographs of the second dataset were used for training an improved neural network for SPHIRE-crYOLO. 191,979 particles were picked with this network on 7,718 micrographs of both datasets and extracted with two-fold binning and a size of 300x300 pixels. An initial 3D refinement of this particle stack in RELION (Zivanov et al., 2019) resulted in a $4.9 \AA$ reconstruction. After several rounds of particle polishing and CTF refinement in RELION, reconstructions with overall nominal resolutions of $4.1 \AA$ and $3.9 \AA$ were obtained using 3D refinement in RELION or nonuniform refinement in cryoSPARC (Punjani et al., 2017; Punjani et al., 2020), respectively (Figure 1 - Supplement 1A-B). Local resolution was calculated, and the reconstruction locally filtered, using cryoSPARC (Figure 1 - Supplement 1C). As the global reconstructions displayed a strong resolution gradient from the center to the exterior parts of the molecule (Figure 1 - Supplement 1C), indicative of continuous flexibility within the complex, we turned to a focused refinement strategy. For this, we generated two focused masks, one for the central and one for the exterior part of one asymmetric half of the molecule. Then, we symmetry-expanded the particle stack according to the C2 symmetry and performed focused local refinements in cryoSPARC that resulted in reconstructions of $3.7 \AA$ and $4.8 \AA$, respectively, for the central and the exterior masks (Figure 1 - Supplement 1A). The maps were fitted to the original unmasked C2-symmetric map, and a composite map created using the 'vop maximum' command in Chimera (Pettersen et al., 2004). 
758 Model building, validation, fitting The central part of RZZ was built de novo using the $3.9 \AA$

759 reconstruction from cryoSPARC non-uniform refinement. This map was subjected to automated

760 model building using phenix.map_to_model (Liebschner et al., 2019). The resulting initial model,

761 which comprised many $\alpha$-helices in the central part of the particle, was manually improved and

762 extended and the correct sequence assigned in Coot (Emsley et al., 2010), yielding a model

763 comprising almost full-length ZW10 and the central part of Rod (residues 890-1440). AlphaFold2

764 (AF2) (Jumper et al., 2021; Tunyasuvunakool et al., 2021) was used in the original implementation 765 as well as in the modified "ColabFold" (Mirdita et al., 2021) and in the "multimer" versions (Evans

766 et al., 2021) to model subcomplexes of RZZ, specifically, the central region of ROD with ZW10,

767 the "hook"-region consisting of the N- and C-termini of two different ROD molecules, the ROD

$768 \beta$-propeller-Zwilch complex and the ROD $\beta$-propeller complex with the C-terminus of Spindly.

769 The overlapping subcomplexes were superimposed, rigid-body-fitted to the RZZ electron density

770 map, and then manually optimized. Flexible dynamic molecular dynamics fitting (Kidmose et al.,

771 2019) and PHENIX real space refinement (Afonine et al., 2013) was employed to refine the fit

772 and optimize the model geometries. In almost all regions, the AF2 predictions explained the

773 electron density map very well after minor alterations, but the choice of the lengths of the

774 interacting fragments of ROD was important, as e.g. the termini of full length ROD are predicted

775 to interact with themselves since the second ROD molecule is missing, and a prediction of the

776 dimeric full length complex was not successful. 


\section{References}

Afonine, P.V., R.W. Grosse-Kunstleve, P.D. Adams, and A. Urzhumtsev. 2013. Bulk-solvent and overall scaling revisited: faster calculations, improved results. Acta Crystallogr D Biol Crystallogr. 69:625-634.

Alfieri, C., S. Zhang, and D. Barford. 2017. Visualizing the complex functions and mechanisms of the anaphase promoting complex/cyclosome (APC/C). Open biology. 7.

Allan, L.A., M. Camacho Reis, G. Ciossani, P.J. Huis In 't Veld, S. Wohlgemuth, G.J. Kops, A. Musacchio, and A.T. Saurin. 2020. Cyclin B1 scaffolds MAD1 at the kinetochore corona to activate the mitotic checkpoint. The EMBO journal. 39:e103180.

Altenfeld, A., S. Wohlgemuth, A. Wehenkel, I.R. Vetter, and A. Musacchio. 2015. Complex assembly, crystallization and preliminary X-ray crystallographic analysis of the human RodZwilch-ZW10 (RZZ) complex. Acta Crystallogr F Struct Biol Commun. 71:438-442.

Arakel, E.C., and B. Schwappach. 2018. Formation of COPI-coated vesicles at a glance. Journal of cell science. 131.

Auckland, P., E. Roscioli, H.L.E. Coker, and A.D. McAinsh. 2020. CENP-F stabilizes kinetochore-microtubule attachments and limits dynein stripping of corona cargoes. The Journal of cell biology. 219.

Barbosa, J., T. Martins, T. Bange, L. Tao, C. Conde, and C. Sunkel. 2020. Polo regulates Spindly to prevent premature stabilization of kinetochore-microtubule attachments. The EMBO journal. 39:e100789.

Barisic, M., B. Sohm, P. Mikolcevic, C. Wandke, V. Rauch, T. Ringer, M. Hess, G. Bonn, and S. Geley. 2010. Spindly/CCDC99 is required for efficient chromosome congression and mitotic checkpoint regulation. Molecular biology of the cell. 21:1968-1981.

Basto, R., R. Gomes, and R.E. Karess. 2000. Rough deal and Zw10 are required for the metaphase checkpoint in Drosophila. Nature cell biology. 2:939-943.

Basto, R., F. Scaerou, S. Mische, E. Wojcik, C. Lefebvre, R. Gomes, T. Hays, and R. Karess. 2004. In vivo dynamics of the rough deal checkpoint protein during Drosophila mitosis. Current biology : CB. 14:56-61.

Brohawn, S.G., N.C. Leksa, E.D. Spear, K.R. Rajashankar, and T.U. Schwartz. 2008. Structural evidence for common ancestry of the nuclear pore complex and vesicle coats. Science. 322:1369-1373.

Buffin, E., C. Lefebvre, J. Huang, M.E. Gagou, and R.E. Karess. 2005. Recruitment of Mad2 to the kinetochore requires the Rod/Zw10 complex. Current biology : CB. 15:856-861. 
Caldas, G.V., T.R. Lynch, R. Anderson, S. Afreen, D. Varma, and J.G. DeLuca. 2015. The RZZ complex requires the $\mathrm{N}$-terminus of KNL1 to mediate optimal Mad1 kinetochore localization in human cells. Open biology. 5.

Chan, Y.W., L.L. Fava, A. Uldschmid, M.H. Schmitz, D.W. Gerlich, E.A. Nigg, and A. Santamaria. 2009. Mitotic control of kinetochore-associated dynein and spindle orientation by human Spindly. The Journal of cell biology. 185:859-874.

Cheerambathur, D.K., R. Gassmann, B. Cook, K. Oegema, and A. Desai. 2013. Crosstalk between microtubule attachment complexes ensures accurate chromosome segregation. Science. 342:1239-1242.

Cianfrocco, M.A., M.E. DeSantis, A.E. Leschziner, and S.L. Reck-Peterson. 2015. Mechanism and regulation of cytoplasmic dynein. Annu Rev Cell Dev Biol. 31:83-108.

Ciossani, G., K. Overlack, A. Petrovic, P.J. Huis In 't Veld, C. Koerner, S. Wohlgemuth, S. Maffini, and A. Musacchio. 2018. The kinetochore proteins CENP-E and CENP-F directly and specifically interact with distinct BUB mitotic checkpoint Ser/Thr kinases. The Journal of biological chemistry. 293:10084-10101.

Civril, F., A. Wehenkel, F.M. Giorgi, S. Santaguida, A. Di Fonzo, G. Grigorean, F.D. Ciccarelli, and A. Musacchio. 2010. Structural analysis of the RZZ complex reveals common ancestry with multisubunit vesicle tethering machinery. Structure. 18:616-626.

Cooke, C.A., B. Schaar, T.J. Yen, and W.C. Earnshaw. 1997. Localization of CENP-E in the fibrous corona and outer plate of mammalian kinetochores from prometaphase through anaphase. Chromosoma. 106:446-455.

De Antoni, A., C.G. Pearson, D. Cimini, J.C. Canman, V. Sala, L. Nezi, M. Mapelli, L. Sironi, M. Faretta, E.D. Salmon, and A. Musacchio. 2005. The Mad1/Mad2 complex as a template for Mad2 activation in the spindle assembly checkpoint. Current biology : CB. 15:214-225.

Emsley, P., B. Lohkamp, W.G. Scott, and K. Cowtan. 2010. Features and development of Coot. Acta Crystallogr D Biol Crystallogr. 66:486-501.

Evans, R., M. O’Neill, A. Pritzel, N. Antropova, A. Senior, T. Green, A. Žídek, R. Bates, S. Blackwell, J. Yim, O. Ronneberger, S. Bodenstein, M. Zielinski, A. Bridgland, A. Potapenko, A. Cowie, K. Tunyasuvunakool, R. Jain, E. Clancy, P. Kohli, J. Jumper, and H. D. 2021. Protein complex prediction with AlphaFold-Multimer. BioRXiv.

Faesen, A.C., M. Thanasoula, S. Maffini, C. Breit, F. Muller, S. van Gerwen, T. Bange, and A. Musacchio. 2017. Basis of catalytic assembly of the mitotic checkpoint complex. Nature. 542:498-502.

Fath, S., J.D. Mancias, X. Bi, and J. Goldberg. 2007. Structure and organization of coat proteins in the COPII cage. Cell. 129:1325-1336. 
Fava, L.L., M. Kaulich, E.A. Nigg, and A. Santamaria. 2011. Probing the in vivo function of Mad1:C-Mad2 in the spindle assembly checkpoint. The EMBO journal. 30:3322-3336.

Fotin, A., Y. Cheng, P. Sliz, N. Grigorieff, S.C. Harrison, T. Kirchhausen, and T. Walz. 2004. Molecular model for a complete clathrin lattice from electron cryomicroscopy. Nature. 432:573-579.

Gama, J.B., C. Pereira, P.A. Simoes, R. Celestino, R.M. Reis, D.J. Barbosa, H.R. Pires, C. Carvalho, J. Amorim, A.X. Carvalho, D.K. Cheerambathur, and R. Gassmann. 2017. Molecular mechanism of dynein recruitment to kinetochores by the Rod-Zw10-Zwilch complex and Spindly. The Journal of cell biology. 216:943-960.

Gassmann, R., A. Essex, J.S. Hu, P.S. Maddox, F. Motegi, A. Sugimoto, S.M. O'Rourke, B. Bowerman, I. McLeod, J.R. Yates, 3rd, K. Oegema, I.M. Cheeseman, and A. Desai. 2008. A new mechanism controlling kinetochore-microtubule interactions revealed by comparison of two dynein-targeting components: SPDL-1 and the Rod/Zwilch/Zw10 complex. Genes \& development. 22:2385-2399.

Gassmann, R., A.J. Holland, D. Varma, X. Wan, F. Civril, D.W. Cleveland, K. Oegema, E.D. Salmon, and A. Desai. 2010. Removal of Spindly from microtubule-attached kinetochores controls spindle checkpoint silencing in human cells. Genes \& development. 24:957-971.

Griffis, E.R., N. Stuurman, and R.D. Vale. 2007. Spindly, a novel protein essential for silencing the spindle assembly checkpoint, recruits dynein to the kinetochore. The Journal of cell biology. 177:1005-1015.

Hauf, S., R.W. Cole, S. LaTerra, C. Zimmer, G. Schnapp, R. Walter, A. Heckel, J. van Meel, C.L. Rieder, and J.M. Peters. 2003. The small molecule Hesperadin reveals a role for Aurora B in correcting kinetochore-microtubule attachment and in maintaining the spindle assembly checkpoint. The Journal of cell biology. 161:281-294.

Henen, M.A., W. Myers, L.R. Schmitt, K.J. Wade, A. Born, P.J. Nichols, and B. Vogeli. 2021. The Disordered Spindly C-terminus Interacts with RZZ Subunits ROD-1 and ZWL-1 in the Kinetochore through the Same Sites in C. Elegans. J Mol Biol. 433:166812.

Hoffman, D.B., C.G. Pearson, T.J. Yen, B.J. Howell, and E.D. Salmon. 2001. Microtubuledependent changes in assembly of microtubule motor proteins and mitotic spindle checkpoint proteins at PtK1 kinetochores. Molecular biology of the cell. 12:1995-2009.

Holland, A.J., R.M. Reis, S. Niessen, C. Pereira, D.A. Andres, H.P. Spielmann, D.W. Cleveland, A. Desai, and R. Gassmann. 2015. Preventing farnesylation of the dynein adaptor Spindly contributes to the mitotic defects caused by farnesyltransferase inhibitors. Molecular biology of the cell. 26:1845-1856.

Hoogenraad, C.C., and A. Akhmanova. 2016. Bicaudal D Family of Motor Adaptors: Linking Dynein Motility to Cargo Binding. Trends Cell Biol. 26:327-340. 
Howell, B.J., B.F. McEwen, J.C. Canman, D.B. Hoffman, E.M. Farrar, C.L. Rieder, and E.D. Salmon. 2001. Cytoplasmic dynein/dynactin drives kinetochore protein transport to the spindle poles and has a role in mitotic spindle checkpoint inactivation. The Journal of cell biology. 155:1159-1172.

Jackman, M., C. Marcozzi, M. Barbiero, M. Pardo, L. Yu, A.L. Tyson, J.S. Choudhary, and J. Pines. 2020. Cyclin B1-Cdk1 facilitates MAD1 release from the nuclear pore to ensure a robust spindle checkpoint. The Journal of cell biology. 219.

Jokelainen, P.T. 1967. The ultrastructure and spatial organization of the metaphase kinetochore in mitotic rat cells. J Ultrastruct Res. 19:19-44.

Jumper, J., R. Evans, A. Pritzel, T. Green, M. Figurnov, O. Ronneberger, K. Tunyasuvunakool, R. Bates, A. Zidek, A. Potapenko, A. Bridgland, C. Meyer, S.A.A. Kohl, A.J. Ballard, A. Cowie, B. Romera-Paredes, S. Nikolov, R. Jain, J. Adler, T. Back, S. Petersen, D. Reiman, E. Clancy, M. Zielinski, M. Steinegger, M. Pacholska, T. Berghammer, S. Bodenstein, D. Silver, O. Vinyals, A.W. Senior, K. Kavukcuoglu, P. Kohli, and D. Hassabis. 2021. Highly accurate protein structure prediction with AlphaFold. Nature. 596:583-589.

Karess, R. 2005. Rod-Zw10-Zwilch: a key player in the spindle checkpoint. Trends Cell Biol. 15:386392.

Katoh, K., K. Misawa, K. Kuma, and T. Miyata. 2002. MAFFT: a novel method for rapid multiple sequence alignment based on fast Fourier transform. Nucleic Acids Res. 30:3059-3066.

Kidmose, R.T., J. Juhl, P. Nissen, T. Boesen, J.L. Karlsen, and B.P. Pedersen. 2019. Namdinator automatic molecular dynamics flexible fitting of structural models into cryo-EM and crystallography experimental maps. IUCrJ. 6:526-531.

Kops, G., and R. Gassmann. 2020. Crowning the Kinetochore: The Fibrous Corona in Chromosome Segregation. Trends Cell Biol. 30:653-667.

Kops, G.J., Y. Kim, B.A. Weaver, Y. Mao, I. McLeod, J.R. Yates, 3rd, M. Tagaya, and D.W. Cleveland. 2005. ZW10 links mitotic checkpoint signaling to the structural kinetochore. The Journal of cell biology. 169:49-60.

Kuhn, J., and S. Dumont. 2017. Spindle assembly checkpoint satisfaction occurs via end-on but not lateral attachments under tension. The Journal of cell biology. 216:1533-1542.

Kuhn, J., and S. Dumont. 2019. Mammalian kinetochores count attached microtubules in a sensitive and switch-like manner. The Journal of cell biology. 218:3583-3596.

Kuijt, T.E.F., M.L.A. Lambers, S. Weterings, B. Ponsioen, A.C.F. Bolhaqueiro, D.H.M. Staijen, and G. Kops. 2020. A Biosensor for the Mitotic Kinase MPS1 Reveals Spatiotemporal Activity Dynamics and Regulation. Current biology : CB. 30:3862-3870 e3866. 
Lara-Gonzalez, P., J. Pines, and A. Desai. 2021. Spindle assembly checkpoint activation and silencing at kinetochores. Semin Cell Dev Biol. 117:86-98.

Lee, C., and J. Goldberg. 2010. Structure of coatomer cage proteins and the relationship among COPI, COPII, and clathrin vesicle coats. Cell. 142:123-132.

Legal, T., D. Hayward, A. Gluszek-Kustusz, E.A. Blackburn, C. Spanos, J. Rappsilber, U. Gruneberg, and J.P.I. Welburn. 2020. The C-terminal helix of BubR1 is essential for CENP-E-dependent chromosome alignment. Journal of cell science. 133.

Liebschner, D., P.V. Afonine, M.L. Baker, G. Bunkoczi, V.B. Chen, T.I. Croll, B. Hintze, L.W. Hung, S. Jain, A.J. McCoy, N.W. Moriarty, R.D. Oeffner, B.K. Poon, M.G. Prisant, R.J. Read, J.S. Richardson, D.C. Richardson, M.D. Sammito, O.V. Sobolev, D.H. Stockwell, T.C. Terwilliger, A.G. Urzhumtsev, L.L. Videau, C.J. Williams, and P.D. Adams. 2019. Macromolecular structure determination using X-rays, neutrons and electrons: recent developments in Phenix. Acta Crystallogr D Struct Biol. 75:861-877.

Magidson, V., R. Paul, N. Yang, J.G. Ault, C.B. O'Connell, I. Tikhonenko, B.F. McEwen, A. Mogilner, and A. Khodjakov. 2015. Adaptive changes in the kinetochore architecture facilitate proper spindle assembly. Nature cell biology.

Matson, D.R., and P.T. Stukenberg. 2014. CENP-I and Aurora B act as a molecular switch that ties RZZ/Mad1 recruitment to kinetochore attachment status. The Journal of cell biology. 205:541-554.

McEwen, B.F., J.T. Arena, J. Frank, and C.L. Rieder. 1993. Structure of the colcemid-treated PtK1 kinetochore outer plate as determined by high voltage electron microscopic tomography. The Journal of cell biology. 120:301-312.

McKenney, R.J., W. Huynh, M.E. Tanenbaum, G. Bhabha, and R.D. Vale. 2014. Activation of cytoplasmic dynein motility by dynactin-cargo adapter complexes. Science. 345:337-341.

Miller, S.A., M.L. Johnson, and P.T. Stukenberg. 2008. Kinetochore attachments require an interaction between unstructured tails on microtubules and Ndc80(Hec1). Current biology : CB. 18:1785-1791.

Mirdita, M., K. Schütze, L. Moriqwaki, S. Ovchinnikov, and M. Steinegger. 2021. ColabFold Making protein folding accessible to all. BioRXiv.

Mische, S., Y. He, L. Ma, M. Li, M. Serr, and T.S. Hays. 2008. Dynein light intermediate chain: an essential subunit that contributes to spindle checkpoint inactivation. Molecular biology of the cell. 19:4918-4929.

Moriya, T., M. Saur, M. Stabrin, F. Merino, H. Voicu, Z. Huang, P.A. Penczek, S. Raunser, and C. Gatsogiannis. 2017. High-resolution Single Particle Analysis from Electron Cryomicroscopy Images Using SPHIRE. $J V$ is Exp. 
Mosalaganti, S., J. Keller, A. Altenfeld, M. Winzker, P. Rombaut, M. Saur, A. Petrovic, A. Wehenkel, S. Wohlgemuth, F. Muller, S. Maffini, T. Bange, F. Herzog, H. Waldmann, S. Raunser, and A. Musacchio. 2017. Structure of the RZZ complex and molecular basis of its interaction with Spindly. The Journal of cell biology. 216:961-981.

Moudgil, D.K., N. Westcott, J.K. Famulski, K. Patel, D. Macdonald, H. Hang, and G.K. Chan. 2015. A novel role of farnesylation in targeting a mitotic checkpoint protein, human Spindly, to kinetochores. The Journal of cell biology. 208:881-896.

Musacchio, A., and A. Desai. 2017. A Molecular View of Kinetochore Assembly and Function. Biology (Basel). 6.

Navarro, A.P., and I.M. Cheeseman. 2021. Kinetochore assembly throughout the cell cycle. Semin Cell Dev Biol. 117:62-74.

Nijenhuis, W., E. von Castelmur, D. Littler, V. De Marco, E. Tromer, M. Vleugel, M.H. van Osch, B. Snel, A. Perrakis, and G.J. Kops. 2013. A TPR domain-containing N-terminal module of MPS1 is required for its kinetochore localization by Aurora B. The Journal of cell biology. 201:217-231.

Pagliuca, C., V.M. Draviam, E. Marco, P.K. Sorger, and P. De Wulf. 2009. Roles for the conserved spc105p/kre28p complex in kinetochore-microtubule binding and the spindle assembly checkpoint. PLoS One. 4:e7640.

Pereira, C., R.M. Reis, J.B. Gama, R. Celestino, D.K. Cheerambathur, A.X. Carvalho, and R. Gassmann. 2018. Self-Assembly of the RZZ Complex into Filaments Drives Kinetochore Expansion in the Absence of Microtubule Attachment. Current biology : CB. 28:3408-3421 e3408.

Pettersen, E.F., T.D. Goddard, C.C. Huang, G.S. Couch, D.M. Greenblatt, E.C. Meng, and T.E. Ferrin. 2004. UCSF Chimera--a visualization system for exploratory research and analysis. J Comput Chem. 25:1605-1612.

Punjani, A., J.L. Rubinstein, D.J. Fleet, and M.A. Brubaker. 2017. cryoSPARC: algorithms for rapid unsupervised cryo-EM structure determination. Nat Methods. 14:290-296.

Punjani, A., H. Zhang, and D.J. Fleet. 2020. Non-uniform refinement: adaptive regularization improves single-particle cryo-EM reconstruction. Nat Methods. 17:1214-1221.

Raaijmakers, J.A., M.E. Tanenbaum, and R.H. Medema. 2013. Systematic dissection of dynein regulators in mitosis. The Journal of cell biology. 201:201-215.

Raaijmakers, J.A., R. van Heesbeen, V.A. Blomen, L.M.E. Janssen, F. van Diemen, T.R. Brummelkamp, and R.H. Medema. 2018. BUB1 Is Essential for the Viability of Human Cells in which the Spindle Assembly Checkpoint Is Compromised. Cell Rep. 22:1424-1438. 
1005

1006

1007

1008

1009

1010

1011

1012

1013

1014

1015

1016

Reck-Peterson, S.L., W.B. Redwine, R.D. Vale, and A.P. Carter. 2018. The cytoplasmic dynein transport machinery and its many cargoes. Nature reviews. Molecular cell biology. 19:382-398.

Rieder, C.L., and S.P. Alexander. 1990. Kinetochores are transported poleward along a single astral microtubule during chromosome attachment to the spindle in newt lung cells. The Journal of cell biology. 110:81-95.

Rodriguez-Rodriguez, J.A., C. Lewis, K.L. McKinley, V. Sikirzhytski, J. Corona, J. Maciejowski, A. Khodjakov, I.M. Cheeseman, and P.V. Jallepalli. 2018. Distinct Roles of RZZ and Bub1KNL1 in Mitotic Checkpoint Signaling and Kinetochore Expansion. Current biology : CB. 28:3422-3429 e3425.

Rohou, A., and N. Grigorieff. 2015. CTFFIND4: Fast and accurate defocus estimation from electron micrographs. J Struct Biol. 192:216-221.

Sacristan, C., M.U.D. Ahmad, J. Keller, J. Fermie, V. Groenewold, E. Tromer, A. Fish, R. Melero, J.M. Carazo, J. Klumperman, A. Musacchio, A. Perrakis, and G.J. Kops. 2018. Dynamic kinetochore size regulation promotes microtubule capture and chromosome biorientation in mitosis. Nature cell biology. 20:800-810.

Santaguida, S., A. Tighe, A.M. D'Alise, S.S. Taylor, and A. Musacchio. 2010. Dissecting the role of MPS1 in chromosome biorientation and the spindle checkpoint through the small molecule inhibitor reversine. The Journal of cell biology. 190:73-87.

Scaerou, F., D.A. Starr, F. Piano, O. Papoulas, R.E. Karess, and M.L. Goldberg. 2001. The ZW10 and Rough Deal checkpoint proteins function together in a large, evolutionarily conserved complex targeted to the kinetochore. Journal of cell science. 114:3103-3114.

Schindelin, J., I. Arganda-Carreras, E. Frise, V. Kaynig, M. Longair, T. Pietzsch, S. Preibisch, C. Rueden, S. Saalfeld, B. Schmid, J.Y. Tinevez, D.J. White, V. Hartenstein, K. Eliceiri, P. Tomancak, and A. Cardona. 2012. Fiji: an open-source platform for biological-image analysis. Nat Methods. 9:676-682.

Schlager, M.A., H.T. Hoang, L. Urnavicius, S.L. Bullock, and A.P. Carter. 2014. In vitro reconstitution of a highly processive recombinant human dynein complex. The EMBO journal. 33:1855-1868.

Sigismund, S., L. Lanzetti, G. Scita, and P.P. Di Fiore. 2021. Endocytosis in the context-dependent regulation of individual and collective cell properties. Nature reviews. Molecular cell biology. 22:625-643.

Silio, V., A.D. McAinsh, and J.B. Millar. 2015. KNL1-Bubs and RZZ Provide Two Separable Pathways for Checkpoint Activation at Human Kinetochores. Developmental cell. 35:600-613.

1017 Simonetta, M., R. Manzoni, R. Mosca, M. Mapelli, L. Massimiliano, M. Vink, B. Novak, A. 1018 Musacchio, and A. Ciliberto. 2009. The influence of catalysis on mad2 activation dynamics. $1019 \quad$ PLoS Biol. 7:e10. 
1030

1031

1032

1033

1034

1035

1036

1037

1038

1039

1040

1041

1042

1043

1044

1045

1046

1047

1048

Sivaram, M.V., T.L. Wadzinski, S.D. Redick, T. Manna, and S.J. Doxsey. 2009. Dynein light intermediate chain 1 is required for progress through the spindle assembly checkpoint. The EMBO journal. 28:902-914.

Stabrin, M., F. Schoenfeld, T. Wagner, S. Pospich, C. Gatsogiannis, and S. Raunser. 2020. TranSPHIRE: automated and feedback-optimized on-the-fly processing for cryo-EM. Nature communications. 11:5716.

Stagg, S.M., C. Gurkan, D.M. Fowler, P. LaPointe, T.R. Foss, C.S. Potter, B. Carragher, and W.E. Balch. 2006. Structure of the Sec13/31 COPII coat cage. Nature. 439:234-238.

Stagg, S.M., P. LaPointe, and W.E. Balch. 2007. Structural design of cage and coat scaffolds that direct membrane traffic. Curr Opin Struct Biol. 17:221-228.

Starr, D.A., B.C. Williams, T.S. Hays, and M.L. Goldberg. 1998. ZW10 helps recruit dynactin and dynein to the kinetochore. The Journal of cell biology. 142:763-774.

Sundin, L.J., G.J. Guimaraes, and J.G. Deluca. 2011. The NDC80 complex proteins Nuf2 and Hec1 make distinct contributions to kinetochore-microtubule attachment in mitosis. Molecular biology of the cell. 22:759-768.

ter Haar, E., A. Musacchio, S.C. Harrison, and T. Kirchhausen. 1998. Atomic structure of clathrin: a beta propeller terminal domain joins an alpha zigzag linker. Cell. 95:563-573.

Tripathi, A., Y. Ren, P.D. Jeffrey, and F.M. Hughson. 2009. Structural characterization of Tip20p and Dsl1p, subunits of the Dsl1p vesicle tethering complex. Nature structural \& molecular biology. 16:114-123.

Tunyasuvunakool, K., J. Adler, Z. Wu, T. Green, M. Zielinski, A. Zidek, A. Bridgland, A. Cowie, C. Meyer, A. Laydon, S. Velankar, G.J. Kleywegt, A. Bateman, R. Evans, A. Pritzel, M. Figurnov, O. Ronneberger, R. Bates, S.A.A. Kohl, A. Potapenko, A.J. Ballard, B. RomeraParedes, S. Nikolov, R. Jain, E. Clancy, D. Reiman, S. Petersen, A.W. Senior, K. Kavukcuoglu, E. Birney, P. Kohli, J. Jumper, and D. Hassabis. 2021. Highly accurate protein structure prediction for the human proteome. Nature. 596:590-596.

Varma, D., P. Monzo, S.A. Stehman, and R.B. Vallee. 2008. Direct role of dynein motor in stable kinetochore-microtubule attachment, orientation, and alignment. The Journal of cell biology. 182:1045-1054.

Varma, D., X. Wan, D. Cheerambathur, R. Gassmann, A. Suzuki, J. Lawrimore, A. Desai, and E.D. Salmon. 2013. Spindle assembly checkpoint proteins are positioned close to core microtubule attachment sites at kinetochores. The Journal of cell biology. 202:735-746.

Vassilev, L.T., C. Tovar, S. Chen, D. Knezevic, X. Zhao, H. Sun, D.C. Heimbrook, and L. Chen. 2006. Selective small-molecule inhibitor reveals critical mitotic functions of human CDK1. Proceedings of the National Academy of Sciences of the United States of America. 103:10660-10665. 
Wagner, T., F. Merino, M. Stabrin, T. Moriya, C. Antoni, A. Apelbaum, P. Hagel, O. Sitsel, T. Raisch, D. Prumbaum, D. Quentin, D. Roderer, S. Tacke, B. Siebolds, E. Schubert, T.R. Shaikh, P. Lill, C. Gatsogiannis, and S. Raunser. 2019. SPHIRE-crYOLO is a fast and accurate fully automated particle picker for cryo-EM. Commun Biol. 2:218.

Watson, E.R., N.G. Brown, J.M. Peters, H. Stark, and B.A. Schulman. 2019. Posing the APC/C E3 Ubiquitin Ligase to Orchestrate Cell Division. Trends Cell Biol. 29:117-134.

Weissmann, F., G. Petzold, R. VanderLinden, P.J. Huis In 't Veld, N.G. Brown, F. Lampert, S. Westermann, H. Stark, B.A. Schulman, and J.M. Peters. 2016. biGBac enables rapid gene assembly for the expression of large multisubunit protein complexes. Proceedings of the National Academy of Sciences of the United States of America. 113:E2564-2569.

Wickham, T.J., T. Davis, R.R. Granados, M.L. Shuler, and H.A. Wood. 1992. Screening of insect cell lines for the production of recombinant proteins and infectious virus in the baculovirus expression system. Biotechnol Prog. 8:391-396.

Williams, B.C., M. Gatti, and M.L. Goldberg. 1996. Bipolar spindle attachments affect redistributions of ZW10, a Drosophila centromere/kinetochore component required for accurate chromosome segregation. The Journal of cell biology. 134:1127-1140.

Wojcik, E., R. Basto, M. Serr, F. Scaerou, R. Karess, and T. Hays. 2001. Kinetochore dynein: its dynamics and role in the transport of the Rough deal checkpoint protein. Nature cell biology. 3:1001-1007.

Wynne, D.J., and H. Funabiki. 2015. Kinetochore function is controlled by a phospho-dependent coexpansion of inner and outer components. The Journal of cell biology. 210:899-916.

Yamamoto, T.G., S. Watanabe, A. Essex, and R. Kitagawa. 2008. SPDL-1 functions as a kinetochore receptor for MDF-1 in Caenorhabditis elegans. The Journal of cell biology. 183:187-194.

Yang, Z., J. Fang, J. Chittuluru, F.J. Asturias, and P.A. Penczek. 2012. Iterative stable alignment and clustering of 2D transmission electron microscope images. Structure. 20:237-247.

Yao, X., K.L. Anderson, and D.W. Cleveland. 1997. The microtubule-dependent motor centromere-associated protein E (CENP-E) is an integral component of kinetochore corona fibers that link centromeres to spindle microtubules. The Journal of cell biology. 139:435-447.

Zhang, G., T. Lischetti, D.G. Hayward, and J. Nilsson. 2015. Distinct domains in Bub1 localize RZZ and BubR1 to kinetochores to regulate the checkpoint. Nature communications. 6:7162.

Zheng, S.Q., E. Palovcak, J.P. Armache, K.A. Verba, Y. Cheng, and D.A. Agard. 2017. MotionCor2: anisotropic correction of beam-induced motion for improved cryo-electron microscopy. Nat Methods. 14:331-332. 
bioRxiv preprint doi: https://doi.org/10.1101/2021.12.03.471119; this version posted December 6, 2021. The copyright holder for this preprint (which was not certified by peer review) is the author/funder, who has granted bioRxiv a license to display the preprint in perpetuity. It is made available under aCC-BY-NC 4.0 International license.

1090 Zivanov, J., T. Nakane, and S.H.W. Scheres. 2019. A Bayesian approach to beam-induced motion

1091 correction in cryo-EM single-particle analysis. IUCrJ. 6:5-17.

1092 
Figure 1. Structural organization of the RZZ complex

1095

(A) Schematic representation of human RZZ subunits. (B) 3D reconstruction of the RZZ complex with densities corresponding to ROD-A, Zwilch-A, and ZW10-A colored in firebrick, yelloworange, and deepblue, respectively. ROD-B, Zwilch-B, and ZW10-B are displayed in equivalent lighter colors as indicated. (C) Cartoon model of the RZZ complex with coloring scheme like in panel A. The position of the internal 2-fold axis of the 2:2:2 hexamer is shown. The $\mathrm{N}$ - and Ctermini of ROD are indicated. Panels B-E and all other panels displaying molecular features were generated with PyMol (The PyMOL Molecular Graphics System, Version 1.2r3pre, Schrödinger, LLC.). (D) 90-degree-rotated view of the complex with linear dimensions. (E) The conservation of residues in an alignment of ROD, Zwilch, and ZW10 is displayed on the surface of the complex

1104 (dark, highly conserved; light, poorly conserved). For all subunits, conservation was calculated 1105 from an alignment of sequences from C. elegans (Ce), D. melanogaster $(\mathrm{Dm}), X$. tropicalis $(\mathrm{Xt})$, D. rerio 1106 (Dr), Bos taurus (Bt), Mus musculus (Mm), and Homo sapiens (Hs). (F) 180-degree-rotated view of the complex. The highest degree of conservation is observed in ZW10. See also alignments in Figure 1 - Supplement 1.

Figure 2. Homo- and heterotypic intermolecular interactions in RZZ

1111 (A) The ROD-A and ROD-B protomers are related by 2 -fold symmetry and interact through two 1112 main regions positioned between residues 485-690 and 1790-2125. (B) A particular prominent 1113 interaction of ROD-A and ROD-B consists in the insertion of the 655-680 loop of one protomer 1114 into a cradle formed by the bending C-terminal region of the second protomer. (C) Zwilch 1115 interacts very prominently with only one of the two ROD protomers, sandwiched between the N1116 terminal $\beta$-propeller and residue 850. A few interactions also link Zwilch to the $\mathrm{C}$-terminal region 1117 of the second protomer. (D) A rotated view shows that ZW10 forms a highly bent, U-shaped 1118 complex with a relatively small inter-protomer interface. (E) The 2-fold axis of the complex 1119 (shown in Figure 1B) crosses the interface between ZW10-A and ZW10-B. (F) Molecular details 1120 of the interface between ZW10-A and ZW10-B with side chains of residues involved.

Figure 3. A farnesyl-binding pocket in the ROD propeller

1123 (A) Schematic representation of HsSpindly. The position of a CC1 box (red) and of the Spindly 1124 box (green) required for binding Dynein:Dynactin are shown, together with the position of 
1125 relevant predicted coiled-coil regions. (B) Cartoon representation of the 7-bladed ROD $\beta$ -

1126 propeller (indicated as blades 1-7). The positions of blade 2, blade 3, and an $\alpha$-helical hairpin 1127 representing an insertion between strands $\beta C 3$ and $\beta \mathrm{D} 3$ are indicated. The four strands of each 1128 blade is indicated as A-D, with A and D being the innermost and outermost strands. The circle 1129 represents the entry point of the farnesyl binding pocket. (C) Surface representation of the ROD $1130 \beta$-propeller. The circle is in the same position shown in panel A. The position of Leu120 is shown 1131 in yellow. This residue was was targeted by photoactivatable crosslinker groups introduced in the 1132 farnesyl group attached to Cys602 of Spindly (Mosalaganti et al., 2017). (D) The position of a 1133 modelled peptide corresponding to the C-terminal region of Spindly as predicted by AlphaFold2 1134 (Jumper et al., 2021; Tunyasuvunakool et al., 2021) with a farnesyl moiety modelled on Cys602. 1135 (E-F) A farnesyl moiety, shown in cyan spheres together with a few C-terminal residues of a 1136 modelled Spindly peptide, fitted snugly into a pocket lined exclusively by the side chains of several 1137 hydrophobic residues. (G) Size-exclusion chromatography profiles and corresponding SDS1138 PAGE of the indicated samples. Two mutant RZZ complexes containing mutations in ROD 1139 L110F-L119F-L120K or I191M are indicated respectively as FFK and I/M. Note that in the 1140 bottom SDS-PAGE the first and second lane were deliberately inverted and contain the first eluted 1141 fraction and the molecular weight marker. For all other shown SDS-PAGE gels, the marker 1142 precedes the first fraction.

\section{Figure 4. MPS1 and Spindly promote corona assembly}

1145 (A) Confocal fluorescence microscopy-based filamentation assay at $561 \mathrm{~nm}$ shows ${ }^{\mathrm{mCh}} \mathrm{RZZS}^{\mathrm{F}}$ (4 $1146 \mu \mathrm{M})$, but not ${ }^{\mathrm{mCh}} \mathrm{RZZ}(4 \mu \mathrm{M})$ or ${ }^{\mathrm{mCh}} \mathrm{S}(4 \mu \mathrm{M})$ as controls, forms filaments at $30^{\circ} \mathrm{C}$. Scale bar: $5 \mu \mathrm{m}$. 1147 (B) Coomassie and ProQ Diamond-stained gels on ${ }^{\mathrm{mCh}}{ }^{\mathrm{RZZS}}{ }^{\mathrm{F}}(4 \mu \mathrm{M})$ treated with the indicated 1148 kinases $(1 \mu \mathrm{M})$ for 15 hours at $20^{\circ} \mathrm{C}$. Rev $=$ reversine, used at $10 \mu \mathrm{M}$. These were precisely the samples studied in experiments in panel C. (C) A filamentation assay as in A demonstrates 1150 sufficiency of MPS1 phosphorylation for filamentation. (D) Levels of Zwilch at kinetochores of 1151 HeLa cells that had been previously synchronized in G2 phase with $9 \mu \mathrm{M}$ RO3306 for $16 \mathrm{~h}$ and 1152 then released into mitosis. Subsequently, cells were immediately treated with $500 \mathrm{nM}$ Reversine, $11533.3 \mu \mathrm{M}$ nocodazole, and $10 \mu \mathrm{M}$ MG132 for 1 hour and imaged while in mitosis. CREST serum 1154 was used to visualize kinetochores and DAPI to stain DNA. Scale bar: $10 \mu \mathrm{m}$. (E) Cells treated as 1155 for panel $\mathbf{D}$ were treated for visualization of Spindly. (F) Scatter dot plots representing normalized 1156 total area of the Zwilch and Spindly signals, normalized to the reversine-untreated control, in the 1157 indicated number of cells from the experiment shown in panels C-D. Red lines indicate mean and 
standard deviation. (G) Representative images showing the effects of a knockdown of the endogenous Spindly in HeLa cells. RNAi treatment was performed for $48 \mathrm{~h}$ with $50 \mathrm{nM}$ siRNA.

1160 Before fixation, cells were synchronized in G2 phase with $9 \mu \mathrm{M}$ RO3306 for $16 \mathrm{~h}$ and then released

1161 into mitosis. Subsequently, cells were immediately treated with $3.3 \mu \mathrm{M}$ nocodazole for an 1162 additional hour. CREST serum was used to visualize kinetochores and DAPI to stain DNA. Scale 1163 bar: $10 \mu \mathrm{m}(\mathbf{H})$ Scatter dot plots representing normalized intensity ratios of Spindly over CREST

1164 for individual kinetochores of cells from the experiment shown in panel G. Red lines indicate 1165 median with interquartile range. (I) Levels of CENP-E and Zwilch were assessed in control cells 1166 and in cells treated as in panel $\mathbf{G}$ to knockdown Spindly. (J) Scatter dot plots representing 1167 normalized total area of the CENP-E and Zwilch signals, normalized to the RNAi negative 1168 control, in the indicated number of cells from the experiment shown in panel I. Red lines indicate mean and standard deviation.

1170

\section{Figure 5 An autoinhibited state of the RZZS complex}

1172 (A) MPS1-induced filamentation experiments demonstrate that the phosphomimetic T13E/S15E 1173 ROD mutant bypasses the filamentation blockade induced by the MPS1 inhibitor reversine. The 1174 T13A/S15A mutant prevents MPS1-induced filamentation altogether. Scale bars in panels A, D, $1175 \mathrm{~F}, \mathrm{G}=5 \mu \mathrm{m}$. (B) The AF2 model confidence score (pLDDT, displayed blue to red through green 1176 from highly to poorly reliable) highlights regions of the model predicted with high or poor 1177 confidence, respectively (Jumper et al., 2021; Tunyasuvunakool et al., 2021). (C) A predicted N1178 terminal extension (green) of the ROD $\beta$-propeller (red), slightly rotated from the view in B. The 1179 propeller proper begins with strand $\beta 7 \mathrm{~d}$ and ends with strand $\beta 7 \mathrm{c}$, which leads into the helical 1180 domain. The N-terminal extension augments the sixth and seventh blades with external $\beta$-strands 1181 ( $\beta 6$ e and $\beta 7$ e). The position of T13 and S15 on the extension is shown. (D) The phosphorylation state of RZZ and Spindly ${ }^{\mathrm{F}}$ was monitored by ProQ Diamond after SDS-PAGE separation of reactions. (E) Filamentation assays with the indicated combinations of $8 \mu \mathrm{M}^{\mathrm{mCh}} \mathrm{RZZS}^{\mathrm{F}}$, MPS1 $(1$

$1184 \mu \mathrm{M})$, and Lambda phosphatase $(0.4 \mathrm{mg} / \mathrm{ml})$ in presence of $10 \mu \mathrm{M}$ reversine. Dephosphorylation reactions were carried out on already formed filaments (see Methods). Samples were imaged by

1186 confocal microscopy. Dephosphorylation does not dissolve already formed filaments. (F) An N1187 terminal deletion mutant of $\operatorname{ROD}(\Delta 15)$ removing the first $15 \mathrm{~N}$-terminal residues that include the 1188 MPS1 phosphorylation sites Thr13 and Ser15 is unable to form filament in presence of MPS1 at $118920^{\circ} \mathrm{C}$, but can form filaments upon mildly heating to $30^{\circ} \mathrm{C}$. (G) RZZ and RZZS complexes were 1190 reconstituted with the Zwilch ${ }^{\mathrm{E} 422 \mathrm{~A} / \mathrm{D} 426 \mathrm{~A}}$ mutant and tested for filamentation at $20^{\circ} \mathrm{C}$ in presence of 
1191 MPS1 or upon mildly heating to $30^{\circ} \mathrm{C}$. These experiments are also displayed in Figure 5 -

1192 Supplement 1A $(\mathbf{H})$ Surface representation of the RZZ model depicting the position of Zwilch ${ }^{\mathrm{E} 422}$ 1193 and Zwilch ${ }^{\mathrm{D} 422}$ (in purple) and the positions of the ROD N-terminal region (green) and the highly 1194 conserved ZW10 N-terminus.

\section{Figure 6 Ultrastructural analysis of RZZS sheets and filaments}

1197 (A) Negative-stain electron microscopy analysis of heat- and MPS1-induced sheets of filament of 1198 farnesylated ${ }^{\mathrm{mCh}} \mathrm{RZZS}$. Scale bar (black): $200 \mathrm{~nm}$. (B) ${ }^{\mathrm{mCh}} \mathrm{RZZ}^{\mathrm{mCh}} \mathrm{S}$ forms rings and curved single 1199 filaments rather than sheets. Scale bar: $200 \mathrm{~nm}$. (C) The GFP of Spindly was removed with 1200 Prescission protease, and the resulting objects were imaged by negative stain EM. Scale bar: 200 nm. (D) Heat- and MPS1-induced rings of ${ }^{\mathrm{mCh}} \mathrm{RZZ} \mathrm{Z}^{\mathrm{mCh}} \mathrm{S}$ have similar diameters.

\section{Figure 7 Influence of Spindly on corona assembly and kinetochore recruitment}

1204 (A) Size-exclusion chromatography and SDS-PAGE of elution fractions of the indicated samples. 1205 Each Spindly construct was incubated with RZZ in absence (dotted lines) or in presence 1206 (continuous lines) of farnesyl transferase and farnesyl pyrophosphate. Elution shifts of Spindly ${ }^{\mathrm{F}}$ and of the resulting RZZS ${ }^{\mathrm{F}}$ complexes is indicative of successful interaction. (B) MPS1-induced 1208 filamentation experiments on the indicated ${ }^{\mathrm{mCh}} \mathrm{RZZS}^{\mathrm{F}}$ complexes $(4 \mu \mathrm{M}$, further diluted to $0.5 \mu \mathrm{M}$ for imaging) using a confocal spinning disk fluorescence microscope at $561 \mathrm{~nm}$. (C) Schematic of the cell synchronization and imaging experiment shown in D. After electroporation, cells were allowed to recover for 8 hours. Subsequently, cells were synchronized in G2 phase with $9 \mu \mathrm{M}$

1212 RO3306 for 15 hours and then released into mitosis by inhibitor washout. Before fixation, cells

1213 were treated with $3.3 \mu \mathrm{M}$ nocodazole for 1 hour. (D) Representative images of fixed HeLa cell 1214 electroporated with full-length Spindly and Spindly ${ }^{354-605}$ constructs in cells depleted of endogenous 1215 Spindly by RNAi. Spindly localization was detected with an antibody against the C-terminal region 1216 of Spindly. Corona expansion or lack thereof were monitored through CENP-E. CREST serum 1217 was used to visualize kinetochores, DAPI to stain for DNA. Scale bar: $10 \mu \mathrm{m}$. (E) Scatter dot plots 1218 representing normalized intensity ratios of the indicated Spindly constructs over CREST for 1219 individual kinetochores of cells from the experiment shown in panel $\mathbf{D}$. Red lines indicate median 1220 with interquartile range. (F) Cells treated like in C-D were electroporated with the indicated ${ }^{\mathrm{mCh}}$ Spindly constructs. Corona expansion was evaluated through the appearance of Zwilch. (G)

1222 Kinetochore intensities were quantified like in panel E. 


\section{Supplemental Figure Legends}

\section{Figure 1 - supplement 1 EM data analyses}

1225 (A) Processing scheme including an exemplary micrograph and a subset of selected 2D classes of

RZZ. (B) Fourier Shell Correlation (FSC) curves of a global (i.e. non-focused) non-uniform 3D refinement in cryoSPARC. The dashed line indicates the 0.143 FSC criterion that intersects the masked FSC curve at $3.94 \AA$. (C) Local resolution plotted on the locally filtered reconstruction obtained by non-uniform refinement in a rainbow-colored gradient from blue (3.7 $\AA$ ) to red $(8.0$ A). (D) Angular distribution displayed as colored bars.

\section{Figure 1 - supplement 2 Multiple sequence alignment of ROD and ZW10}

ROD and ZW10 sequences from Caenorhabditis elegans (Ce), Drosopbila melanogaster (Dm), Xenopus tropicalis (Xt), Danio rerio (Dr), Bos taurus (Bt), Mus musculus (Mm), and Homo sapiens (Hs) were aligned with MAFFT (Katoh et al., 2002) and visualized with software developed in house. The secondary structure of the two RZZ subunits (straight black lines on green, $\beta$-strands; loopy black lines on red, helices; grey, coils) is displayed above the aligned sequences. For ROD, note in the second row a helical hairpin discussed in the text that lines the farnesyl-binding cavity, and the corresponding short deletions in species (Ce and Dm) where Spindly is not farnesylated.

Figure 2 - Supplement 1 Comparison of structural homologs of RZZ subunits

(A) Cartoon model of ROD and of the indicated proteins that share the same structural cryo-EM reconstruction (yellow) and of the previously published crystal structure of Zwilch (green) (Civril et al., 2010). (C) Cartoon model of ZW10 and superposition with Tip20, a related

1246 S. cerevisiae's ortholog. The interdomain angle of the $\mathrm{N}$ - and C-terminal domains is very different 1247 in the two structures.

Figure 3 - Supplement 1 Comparison of the HsROD and CeROD $\beta$-propellers

1250 (A) The $\beta$-propeller of human ROD already shown in Figure 3B with the indicated farnesyl1251 binding site. (B) $\beta$-propeller of C. elegans' ROD predicted by AlphaFold2 (Jumper et al., 2021;

1252 Tunyasuvunakool et al., 2021). (C-D) Modelled farnesyl groups in the two structures demonstrate 
occlusion of the binding cavity in C. elegans, where M184 is predicted to sterically clash with a hypothetical farnesyl moiety.

Figure 4 - Supplement 1 Additional polymerization and cell biology experiments

1257

(A-B) Assessment of requirements for MPS1 phosphorylation for corona assembly in vitro. Samples 1-8 are shown on the left in Coomassie-stained and ProQ Diamond-stained SDS-PAGE. The content of samples 1-8 is described in the legend under panel A, and additionally for samples 2-5 over each polymerization experiment in panel $\mathrm{B}$, where the encircled $\mathrm{P}$ signals which sample was pre-phosphorylated with MPS1 and which samples were treated with reversine to inhibit 1262 MPS1. In B, samples were imaged in a spinning disk confocal microscope at $561 \mathrm{~nm}$. Scale bar = $12635 \mu \mathrm{m}$. (C) Heat-induced polymerization experiments with ${ }^{\mathrm{mCh}} \mathrm{RZZ}$ and wild-type Spindly ${ }^{\mathrm{F}}$ or

1264 Spindly ${ }^{\mathrm{F}(\mathrm{C} 602 \mathrm{~A})}$ that cannot be farnesylated. The same positive control is also shown in Figure 7 1265 Supplement 1B. (D) Levels of BUB1 at kinetochores of HeLa cells that had been previously 1266 synchronized in G2 phase with $9 \mu \mathrm{M}$ RO3306 for $16 \mathrm{~h}$ and then released into mitosis. 1267 Subsequently, cells were immediately treated with $500 \mathrm{nM}$ reversine, $3.3 \mu \mathrm{M}$ nocodazole, and 10 $\mu \mathrm{M}$ MG132 for 1 hour. CREST serum was used to visualize kinetochores and DAPI to stain DNA. Scale bar: $10 \mu \mathrm{m}$. (E) Scatter dot plots representing normalized intensity ratios of BUB1 over CREST for individual kinetochores of cells from the experiment shown in panel C. Red lines indicate median with interquartile range. (F) HeLa cells were synchronized in G2 phase with $9 \mu \mathrm{M}$ RO3306 for 16 hours and the released into mitosis by withdrawing the inhibitor. Cells were immediately treated with $3.3 \mu \mathrm{M}$ nocodazole to prevent microtubule depolymerization and allow maximal corona expansion. After one hour, cells were treated again with RO3306 for the indicated time points, before fixation and further processed for fluorescence microscopy. CREST serum was used to visualize kinetochores and DAPI to visualize DNA. Scale bar: $10 \mu \mathrm{m}$. (G) Quantification of the experiment in panel E. (H) DLD-1 cells treated like in panel E were released from the $\mathrm{G} 2$ arrest into mitosis for 1 hour in presence of $3.3 \mu \mathrm{M}$ nocodazole, $500 \mathrm{nM}$ hesperadine, and $10 \mu \mathrm{M}$ MG132, fixed, and further processed for fluorescence microscopy.

\section{Figure 5 - Supplement 1 Additional in vitro polymerization and biochemical experiments}

1282 (A) The first two rows of experiments are already displayed as the two rows of Figure 5A. The 1283 third row is added to demonstrate that the ${ }^{\mathrm{mCh}} \mathrm{RZZS}^{\mathrm{F}}$ complex carrying T13A/S15A mutations on 1284 ROD, which does not spontaneously filament in presence of MPS1 at $20^{\circ} \mathrm{C}$, will form filaments upon mild heating at $30^{\circ} \mathrm{C}$, indicating that its ability to polymerize is not compromised. Scale bar: 
$12865 \mu \mathrm{M}$. (B) None of the indicated samples forms filaments in absence of Spindly ${ }^{\mathrm{F}}$. Scale bar: $5 \mu \mathrm{m}$.

1287 (C) Size-exclusion chromatography experiment demonstrating that RZZ complex carrying

1288 E422A/D426A mutations interacts with Spindly ${ }^{\mathrm{F}}$ as strongly as its wild type counterpart.

1289

$1290 \quad$ Figure 6-Supplement 1

1291 (A) Untagged RZZS ${ }^{\mathrm{F}}$ formed sheets indistinguishable from those formed by ${ }^{\mathrm{mCh}}{ }^{\mathrm{R} Z Z S^{\mathrm{F}}}$. Scale bar

1292 (black line): $200 \mathrm{~nm}$. (B) Sheets were also formed by ${ }^{\mathrm{mCh}}{ }^{\mathrm{R} Z Z \mathrm{~S}^{\mathrm{F}}}$ containing the T13A/S15A

1293 mutations on ROD upon heating the sample to $30^{\circ} \mathrm{C}$. The phosphomimetic mutant T13E/S15E

1294 of the same complex assembled into filaments spontaneously at $20^{\circ} \mathrm{C}$. The ${ }^{\mathrm{mCh}-\Delta 15} \mathrm{RZZS}^{\mathrm{F}}$ forms

1295 sheets at $30^{\circ} \mathrm{C}$. Scale bar (black line): $200 \mathrm{~nm}$. (C) ${ }^{\mathrm{mCh}} \mathrm{RZZ} /{ }^{\mathrm{GFP}} \mathrm{S}^{\mathrm{F}}$ formed ring structures or curved

1296 filaments similar to those observed with ${ }^{\mathrm{mCh}} \mathrm{RZZ} /{ }^{\mathrm{mCh}} \mathrm{S}^{\mathrm{F}}$. (D) $2 \mathrm{D}$ class averages of segments of

1297 negatively-stained single circles or filaments like those shown in panel C. Note the extreme

1298 orientation preference of the various classes. Scale bar: $50 \mathrm{~nm}$. (E) Enlargement of one class

1299 average shown in $\mathrm{D}$ with indicated dimensions. (F) A size comparison from a field of ${ }^{\mathrm{mCh}} \mathrm{RZZ}^{\mathrm{mCh}} \mathrm{S}^{\mathrm{F}}$

1300 and ${ }^{\text {GFP }}$ CENP-E at kinetochore coronas of nocodazole-treated HeLa cells is shown ad the same

1301 magnification to emphasize the similarity of curvatures in rings and coronas.

1302

1303 Figure 7 - Supplement 1 Additional biochemical experiments

1304 (A) Heat-induced filaments of the indicated species. Note that Spindly $\mathrm{F}^{\mathrm{F}(250-605)}$ and Spindly $\mathrm{F}^{\mathrm{F}(\Delta 276-306)}$ 1305 may show a combination of slight precipitation and filamentation when filamentation is induced 1306 with heat. The same positive control is also shown in Figure 5 - Supplement 1D. (B) Size-exclusion 1307 chromatography of Spindly ${ }^{\mathrm{F}(\Delta 276-306)}, \mathrm{RZZ}$, and their complex. $(\mathbf{C})$ Titration of $^{\mathrm{mCh}}{ }^{\mathrm{RZZ}}$ and Spindly ${ }^{\mathrm{F}}$ 1308 at the indicated concentrations. 

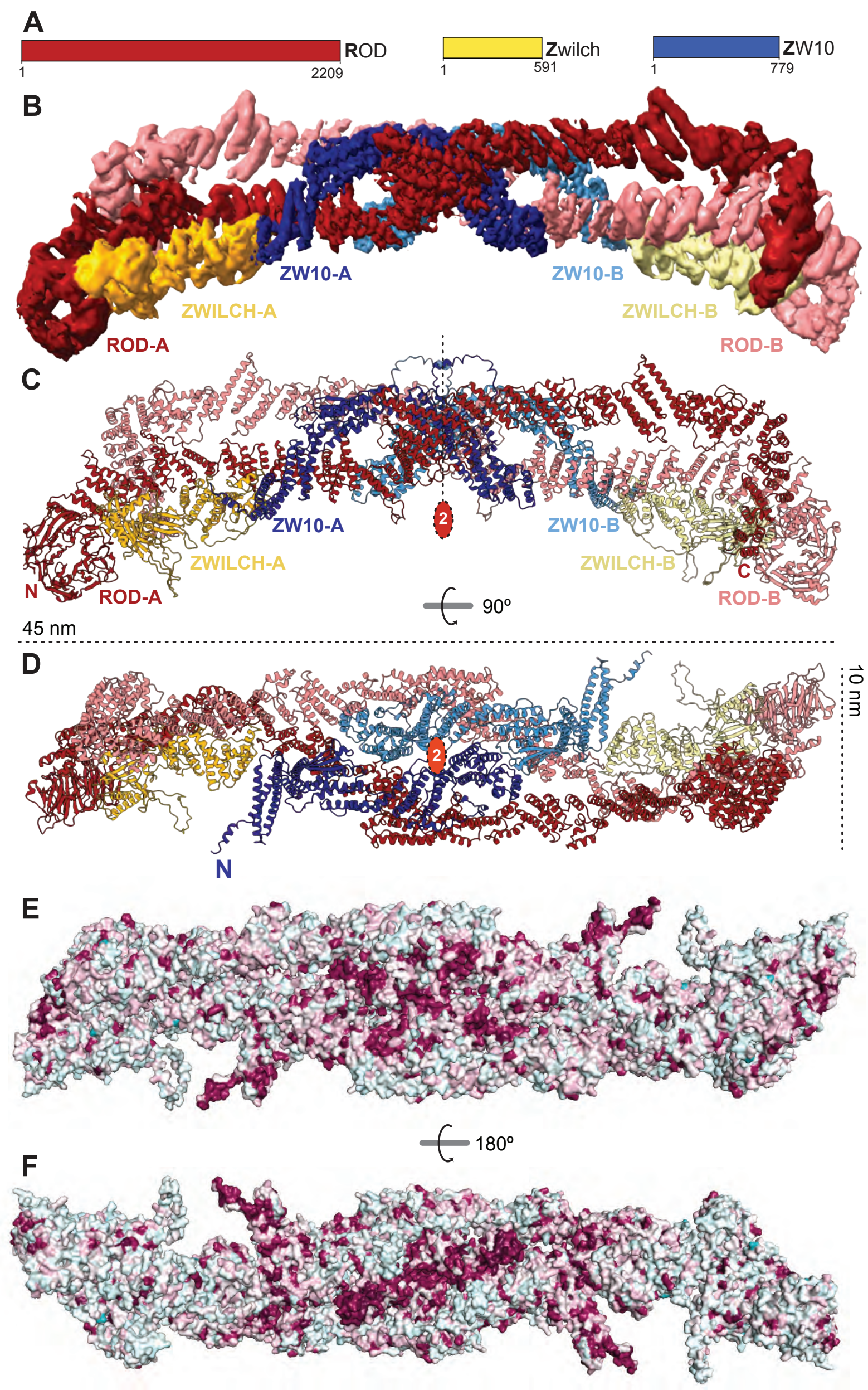
bioRxiv preprint doi: https://doi org/10.1101/2021.12.03.471119. this version posted December 6, 2021. The copyright holder for this

preprint (which was not certified by peer review) is the author/funder, who has granted bioRxiv a license to display the preprint in perpetuity. It is made available under aCC-BY-NC 4.0 International license.
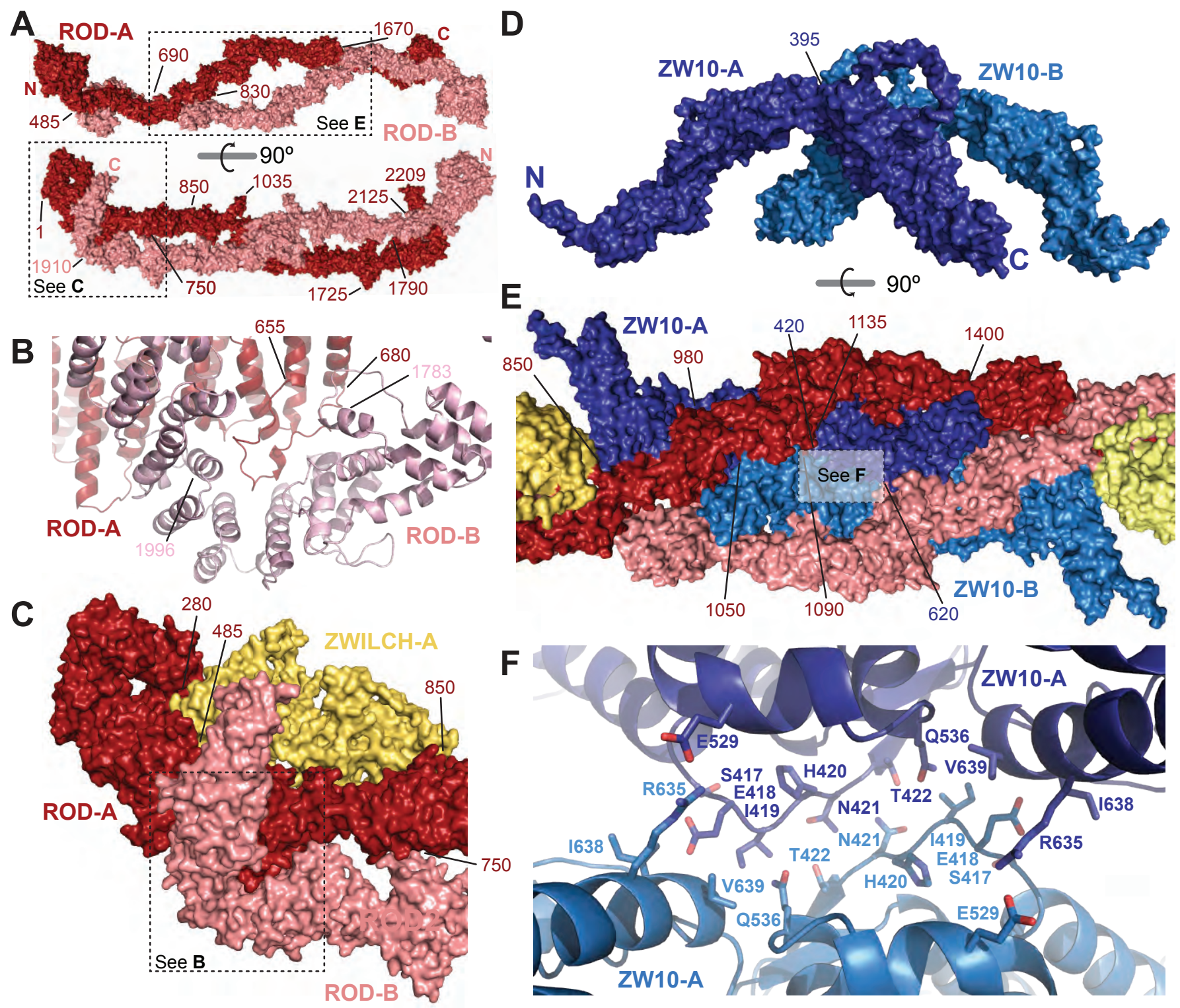

Figure 2 
bioRxiv preprint doi: https://doi.org/10.1101/2021.12.03.471119; this version posted December 6, 2021. The copyright holder for this preprint (which was not certified by peer review) is the author/funder, who has granted bioRxiv a license to display the preprint in perpetuity. It is made available under aCC-BY-NC 4.0 International license.
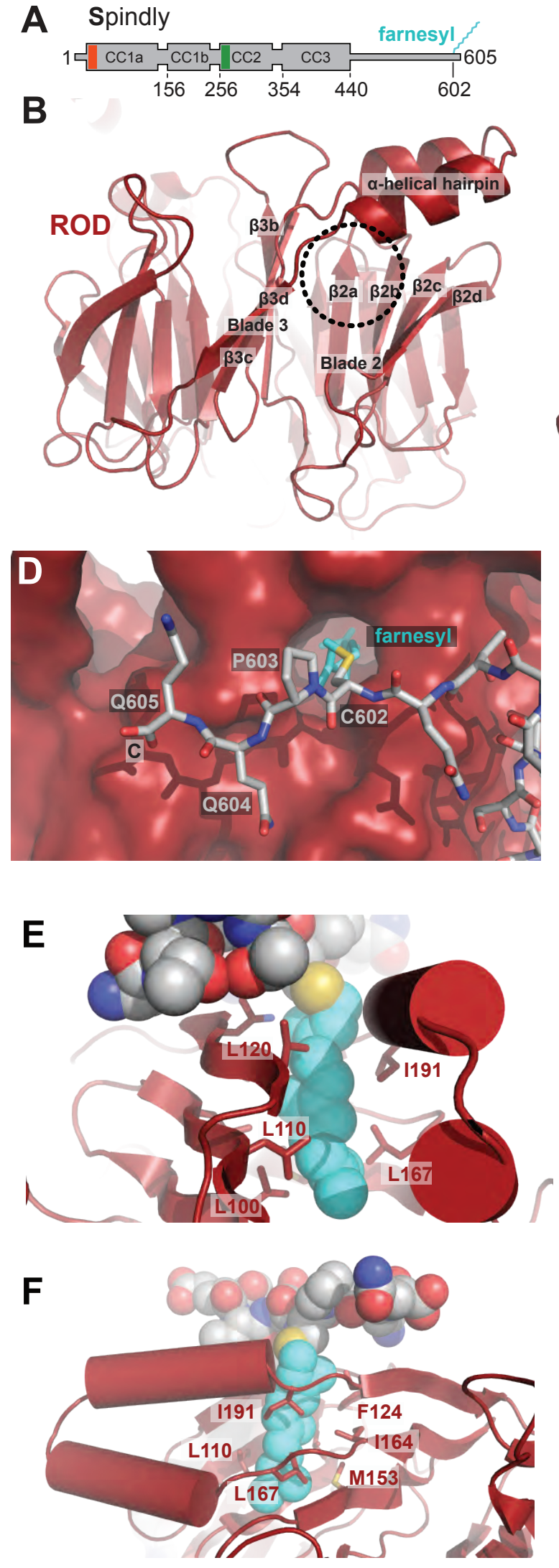

C

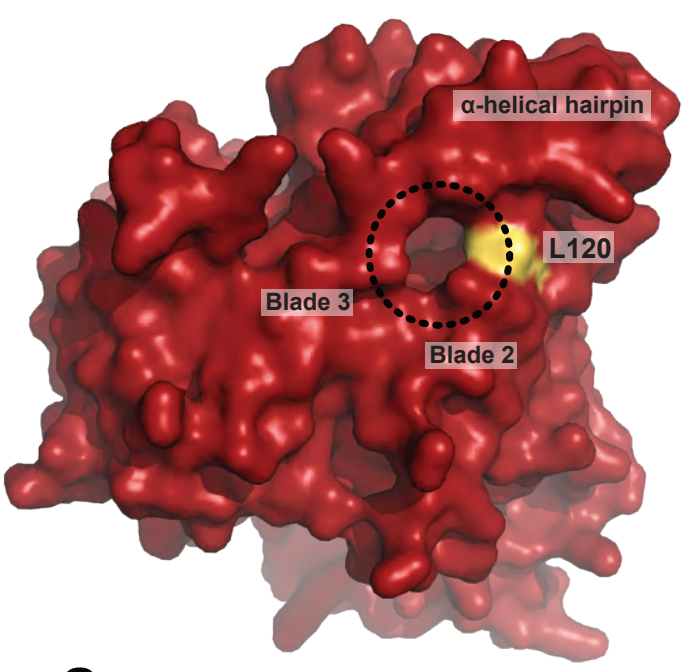

G
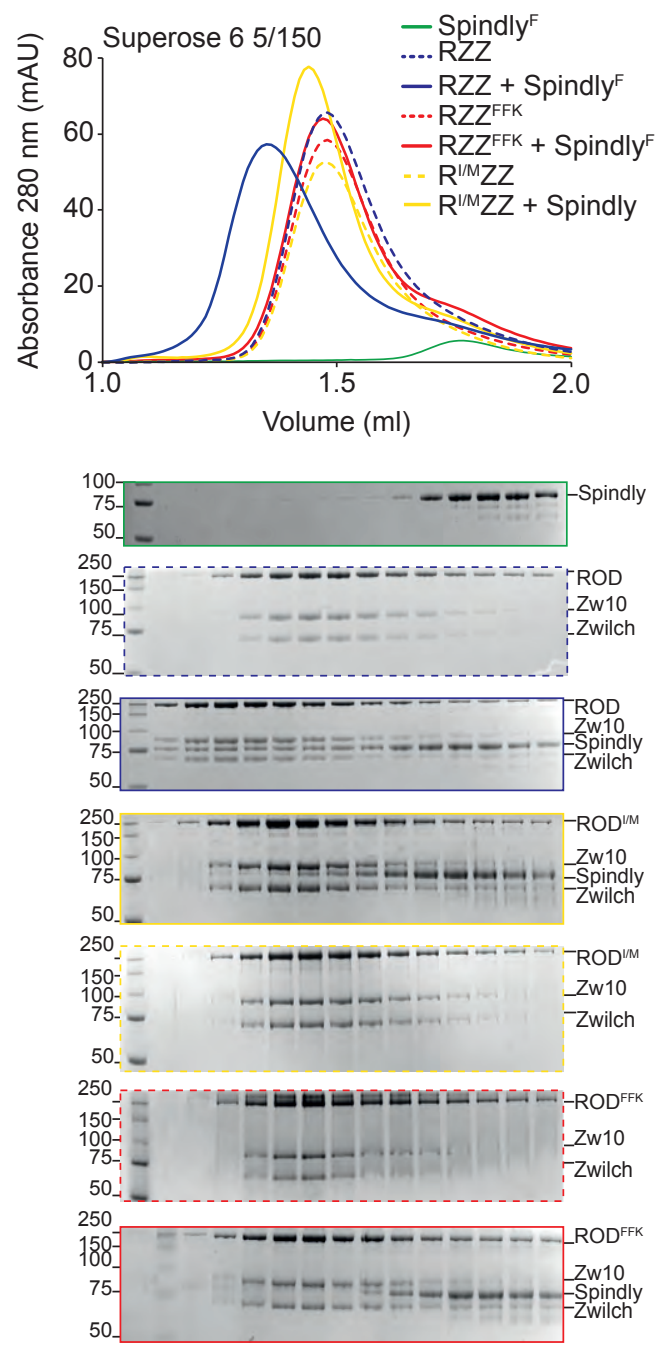
bioRxiv preprint doi: https://doi.org/10.1101/2021.12.03.471119; this version posted December 6, 2021. The copyright holder for this

preprint (which was not certified by peer review) is the author/funder, who has granted bioRxiv a license to display the preprint in perpetuity. It is made available under aCC-BY-NC 4.0 International license.
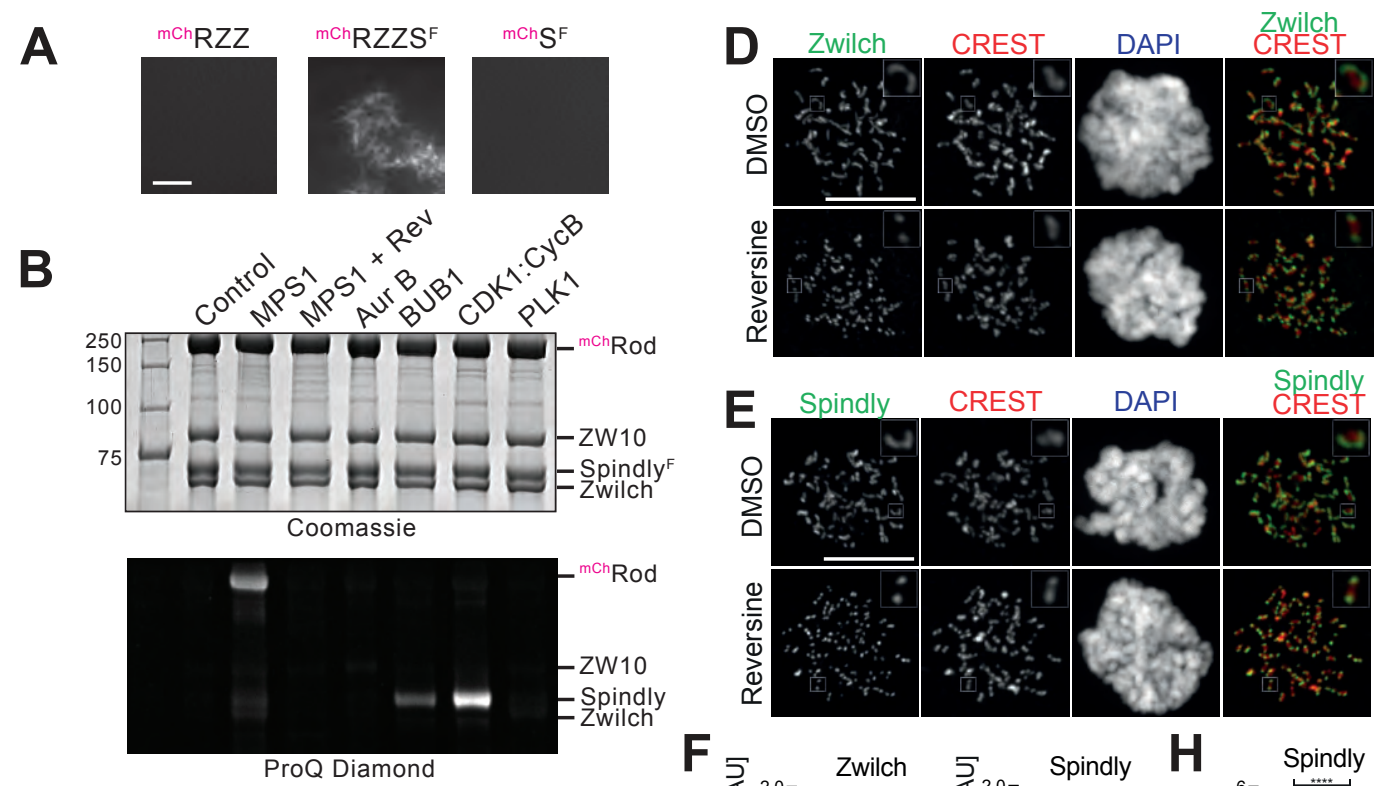

C
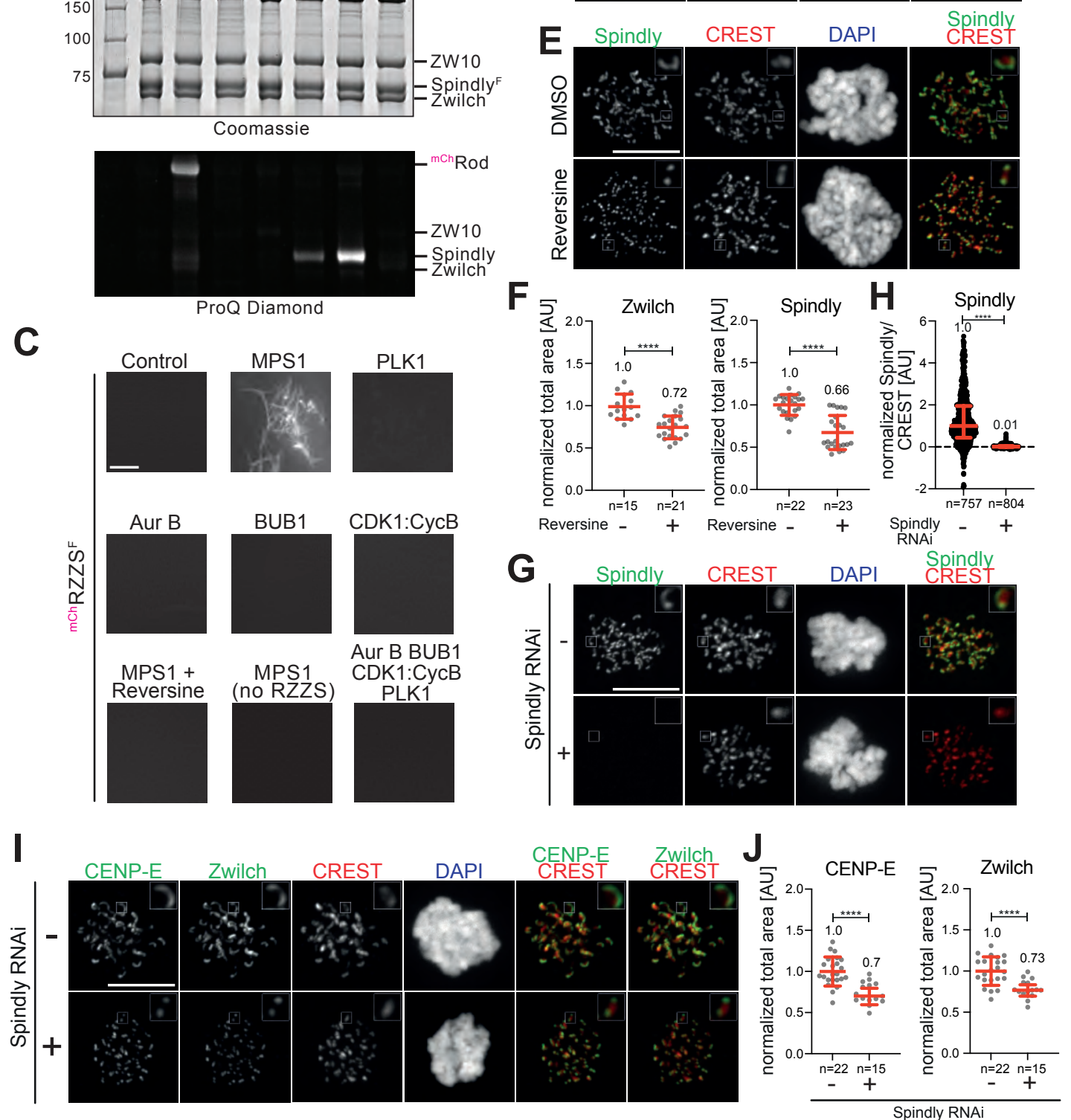

Figure 4 
bioRxiv preprint doi: https://doi org/10.1101/2021.12 03.471119. this version posted December 6, 2021. The copyright holder for this
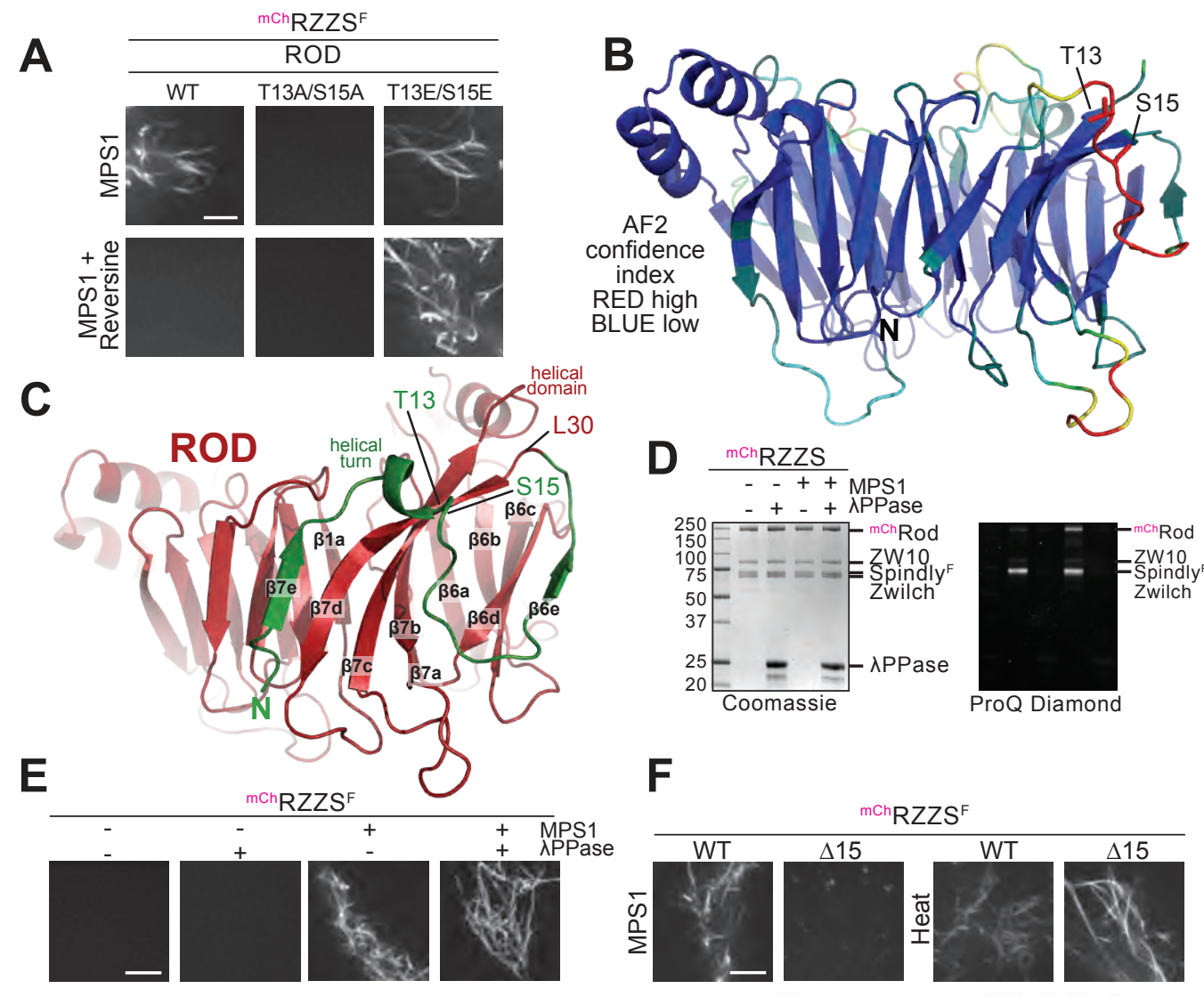

D ${ }^{\mathrm{mch} R Z Z S}$

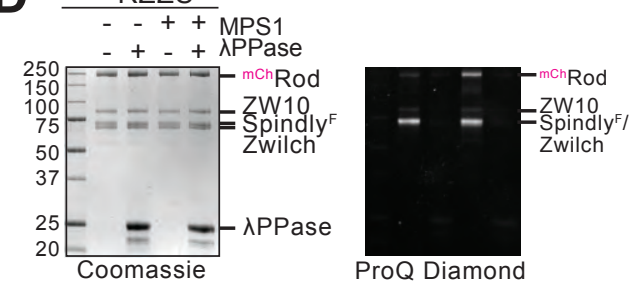

F

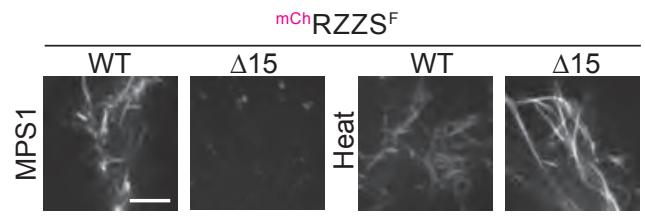

G
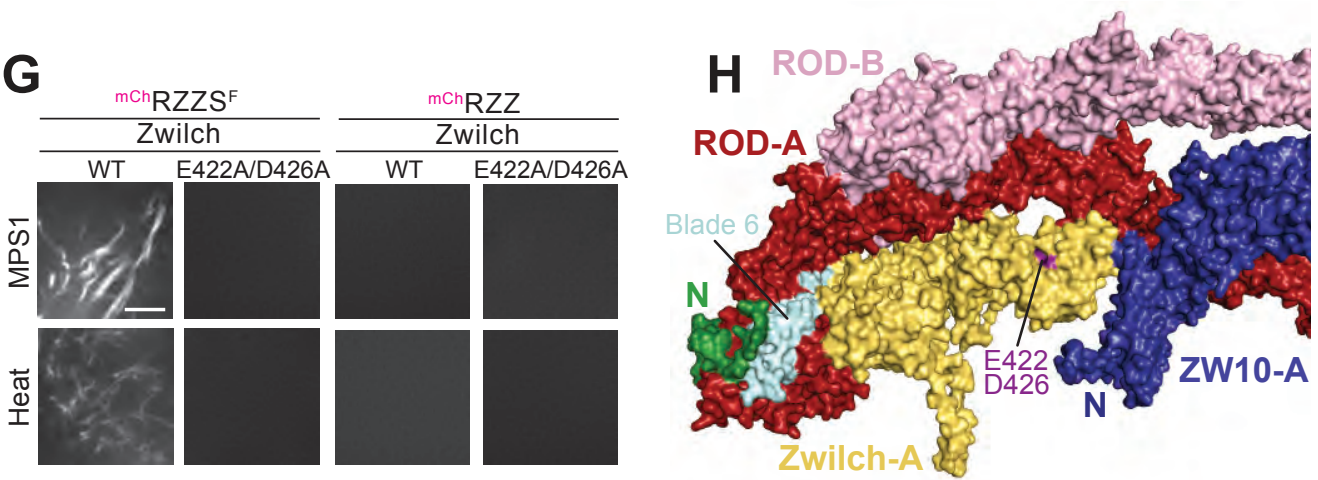

Figure 5 
bioRxiv preprint doi: https://doi.org/10.1101/2021.12.03.471119; this version posted December 6, 2021. The copyright holder for this preprint (which was not certified by peer review) is the author/funder, who has granted bioRxiv a license to display the preprint in perpetuity. It is made available under aCC-BY-NC 4.0 International license.

A

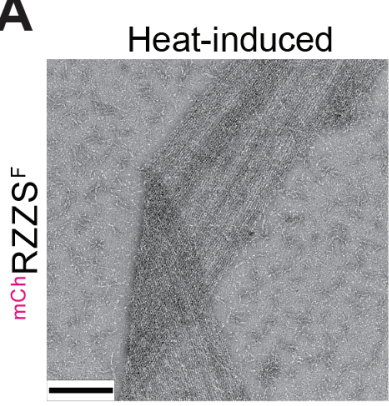

\section{MPS1-induced}
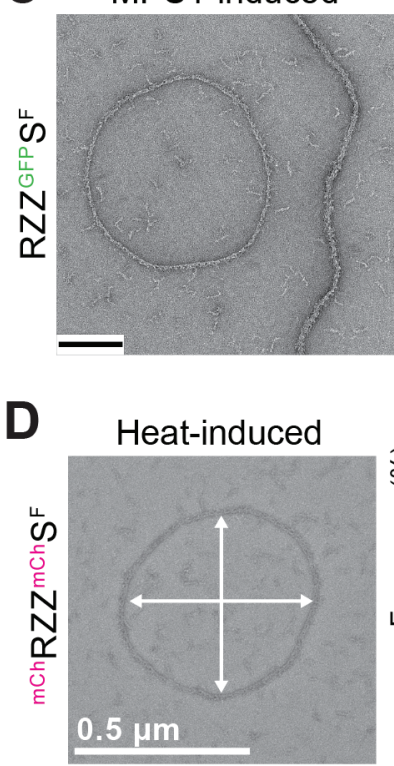

MPS1-induced

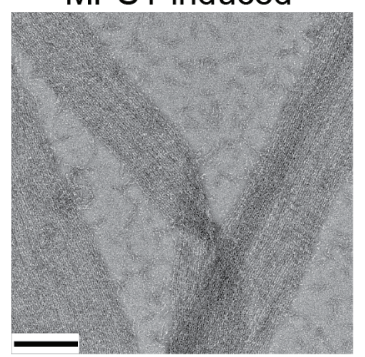

Heat-induced
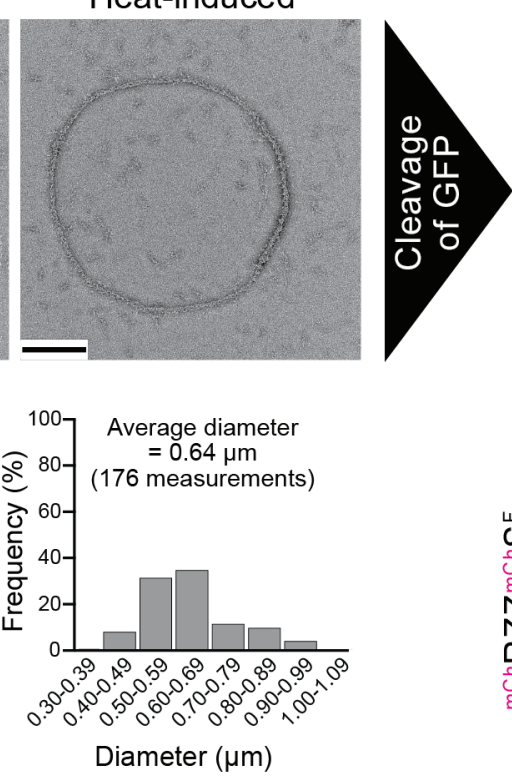

B
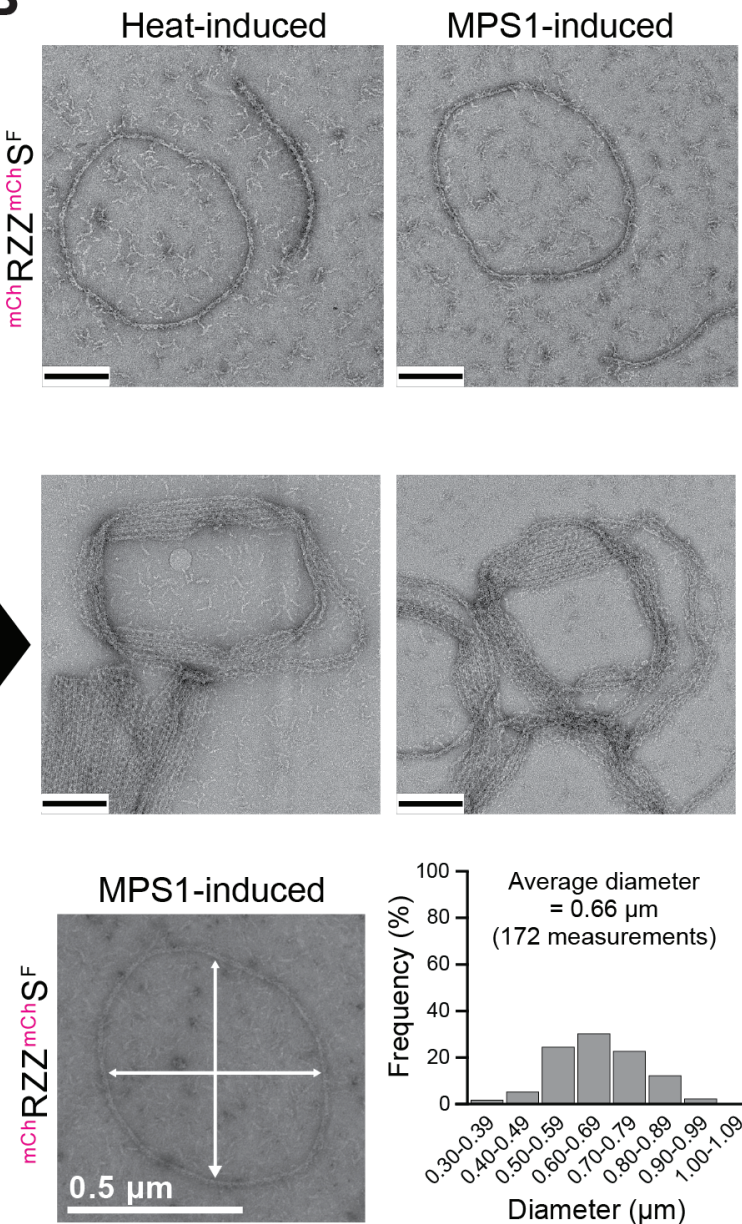

Figure 6 
A
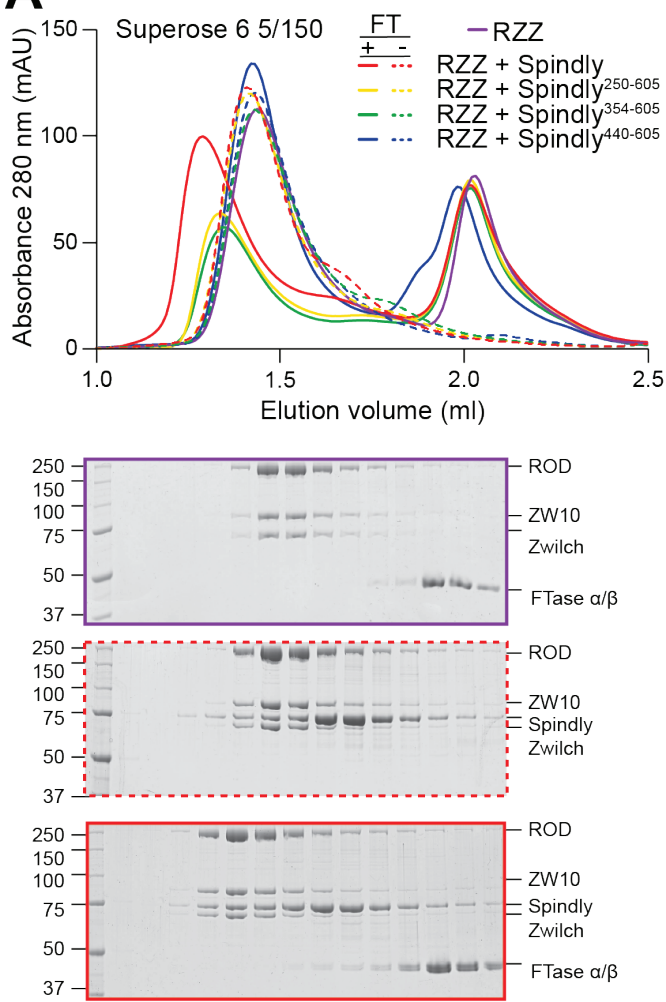

250 -

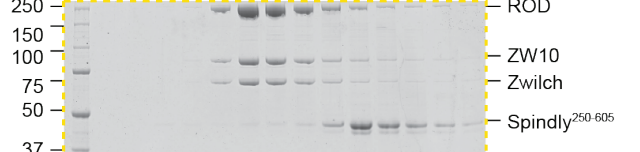

$250-$
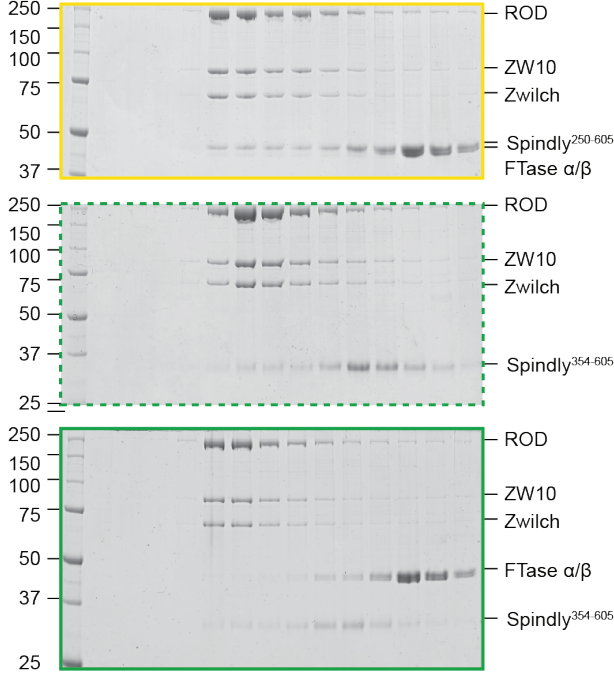

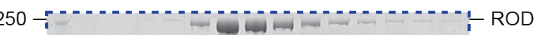

150

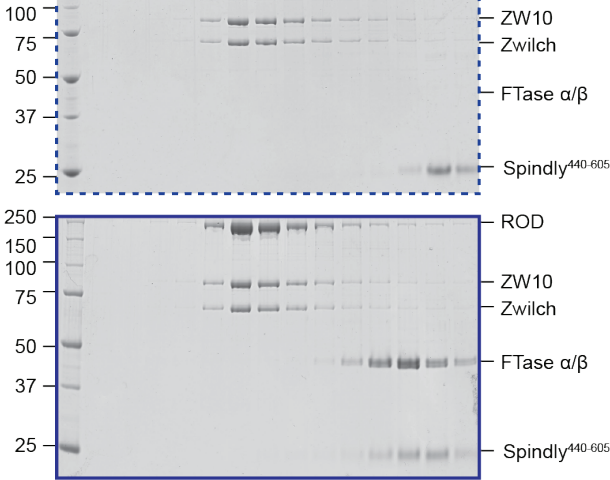

B

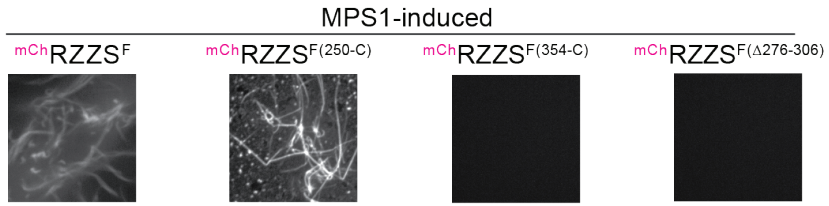

C

Seed cells, $50 \mathrm{nM}$
Spindly'RNAi

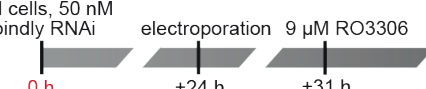
RO3306 wash out
$3.3 \mu \mathrm{M}$ Nocodazole

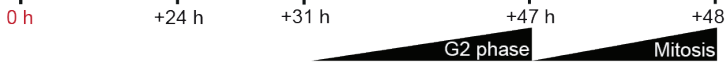

G2 phase

D Spindly
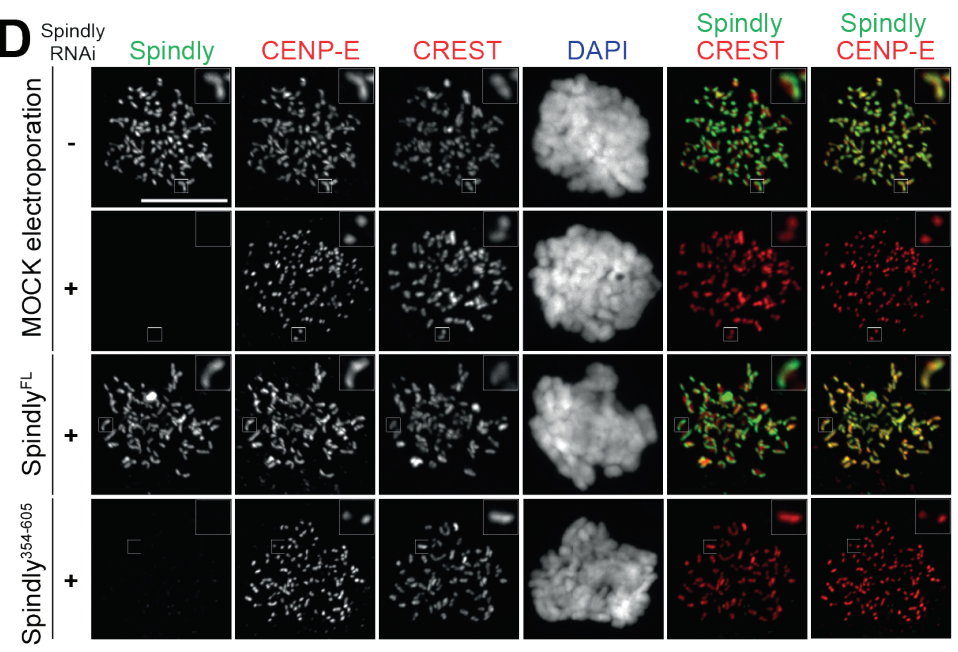

E

$\mathbf{G}$
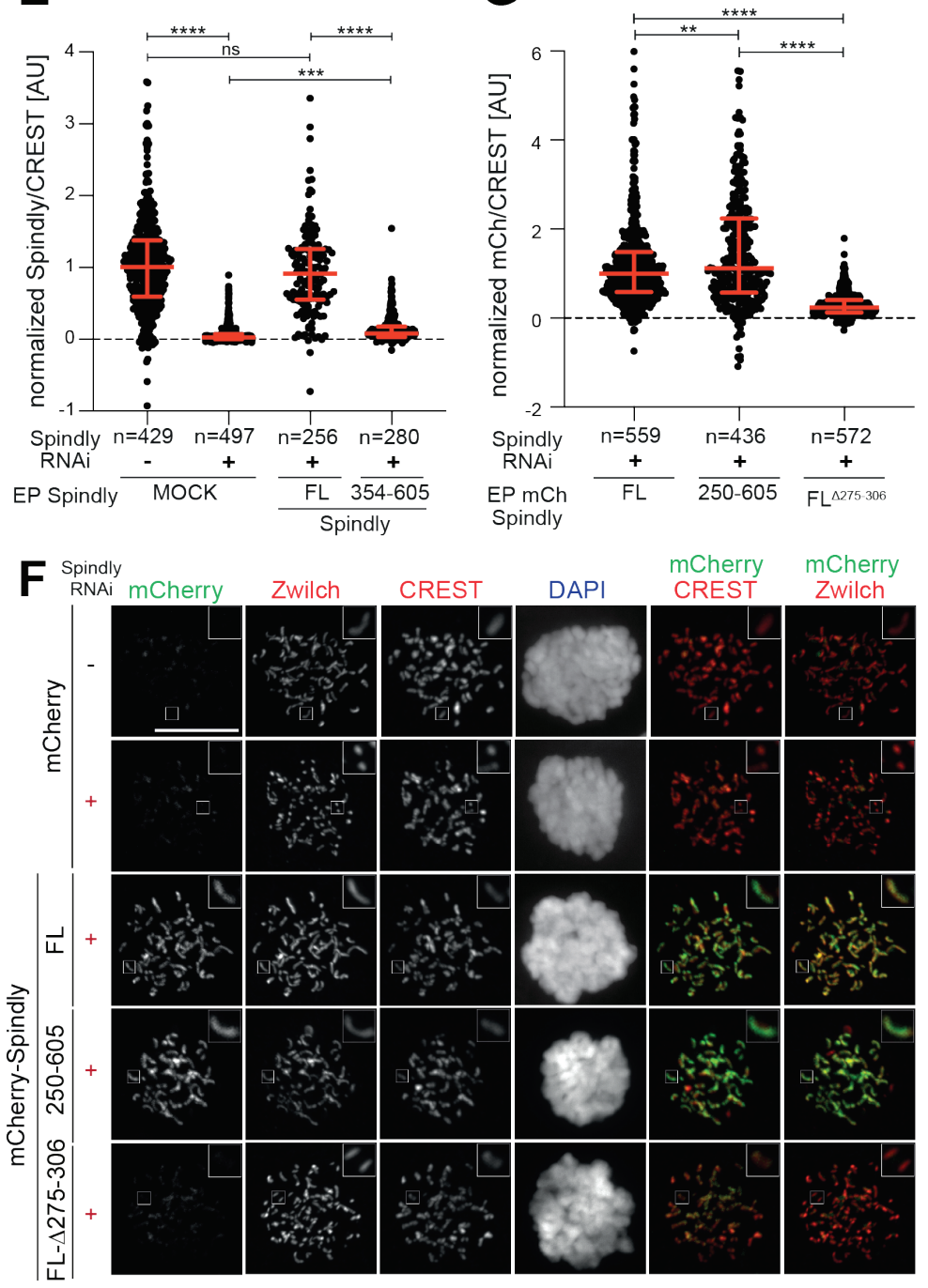

Figure 7 
bioRxiv preprint doi: https://doi.org/10.1101/2021.12 03.471119; this version posted December 6, 2021. The copyright holder for this preprint (which was not certified by peer review) is the author/funder, who has granted bioRxiv a license to display the preprint in perpetuity. It is made available under aCC-BY-NC 4.0 International license.

Supplementary Table 1. Cryo-EM data collection, refinement and validation statistics

\begin{tabular}{|c|c|}
\hline Data collection and processing & \\
\hline Magnification & 105,000 \\
\hline Voltage $(\mathrm{kV})$ & 300 \\
\hline Electron exposure $\left(\mathrm{e}-/ \AA^{2}\right)$ & $59-60$ \\
\hline Defocus range $(\mu \mathrm{m})$ & -1.2 to -2.7 \\
\hline Pixel size $(\AA)$ & 0.9 \\
\hline Symmetry imposed & $\mathrm{C} 2$ \\
\hline Initial particle images (no.) & 275,492 \\
\hline Final particle images (no.) & 191,979 \\
\hline Map resolution $(\AA)$ & 3.9 \\
\hline FSC threshold & 0.143 \\
\hline Map resolution range $(\AA)$ & $3.7-8.0$ \\
\hline \multicolumn{2}{|l|}{ Refinement } \\
\hline \multicolumn{2}{|l|}{ Initial model used (PDB code) } \\
\hline \multicolumn{2}{|l|}{ Model resolution $(\AA)$} \\
\hline FSC threshold & 0.5 \\
\hline \multicolumn{2}{|l|}{ Map sharpening B factor $\left(\AA^{2}\right)$} \\
\hline \multicolumn{2}{|l|}{ Model composition } \\
\hline \multicolumn{2}{|l|}{ Non-hydrogen atoms } \\
\hline \multicolumn{2}{|l|}{ Protein residues } \\
\hline \multicolumn{2}{|l|}{ Ligands } \\
\hline \multicolumn{2}{|l|}{ Water } \\
\hline \multicolumn{2}{|l|}{ B factors $\left(\AA^{2}\right)$} \\
\hline \multicolumn{2}{|l|}{ Protein } \\
\hline Ligand & \\
\hline
\end{tabular}


bioRxiv preprint doi: https://doi org/10.1101/2021.12.03.471119; this version posted December 6, 2021. The copyright holder for this preprint (which was not certified by peer review) is the author/funder, who has granted bioRxiv a license to display the preprint in perpetuity. It is made available under aCC-BY-NC 4.0 International license.

\begin{tabular}{|c|l|}
\hline Water & \\
\hline R.m.s. deviations & \\
\hline Bond lengths $(\AA)$ & \\
\hline Bond angles $(\%)$ & \\
\hline Validation & \\
\hline MolProbity score & \\
\hline Clashscore & \\
\hline Poor rotamers $(\%)$ & \\
\hline Ramachandran plot & \\
\hline Favored $(\%)$ & \\
\hline Allowed $(\%)$ & \\
\hline Disallowed $(\%)$ & \\
\hline
\end{tabular}


bioRxiv preprint doi: https://doi.org/10.1101/2021.12 03.471119; this version posted December 6, 2021. The copyright holder for this preprint (which was not certified by peer review) is the author/funder, who has granted bioRxiv a license to display the preprint in perpetuity. It is made available under aCC-BY-NC 4.0 International license.

\section{A Processing}
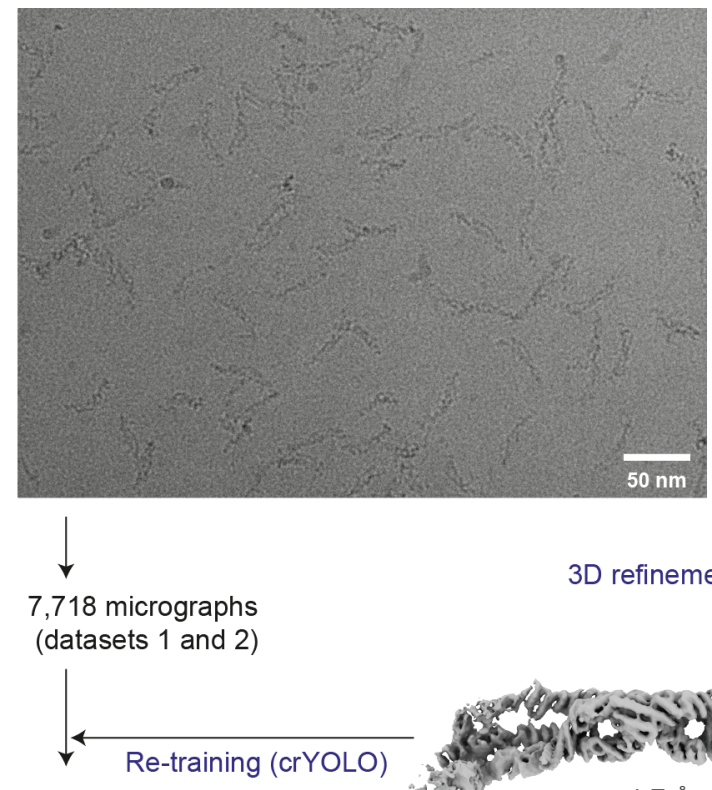

191,826 particles

$4.7 \AA$
$1,968$ micrographs (dataset 1$)$ 74,836 particles

5,794 micrographs (dataset 2) 200,460 particles

$\downarrow 2 \mathrm{D}$ classification

49,656 particles

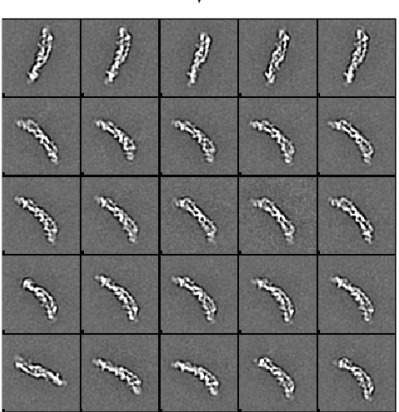

Ab initio model (RVIPER)

3D refinement (MERIDIEN)

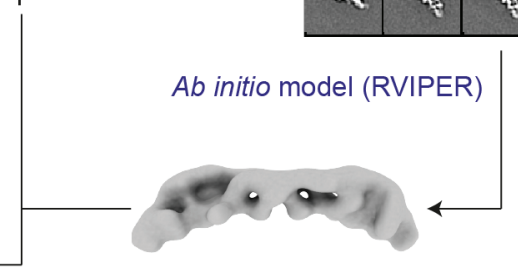

$3 D$ refinement (RELION)

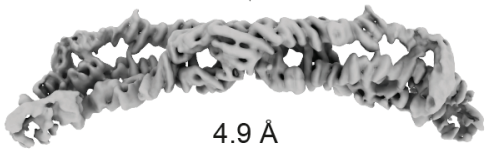

CTF refinement

$\downarrow$ Bayesian polishing (RELION)

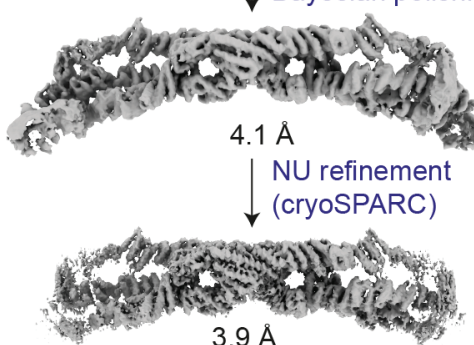

$3.9 \AA$

Symmetry expansion (C2)

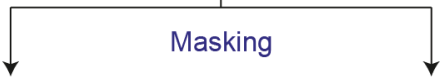

Э-

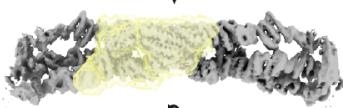

$\vartheta$

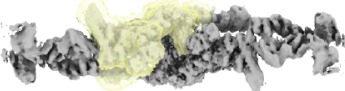

$\downarrow$ Local refinement (cryoSPARC) $\downarrow$

\section{antert}

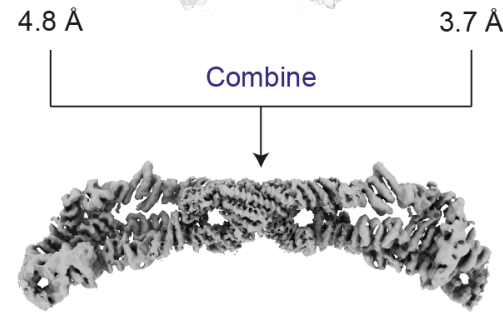

\section{B FSC curve}

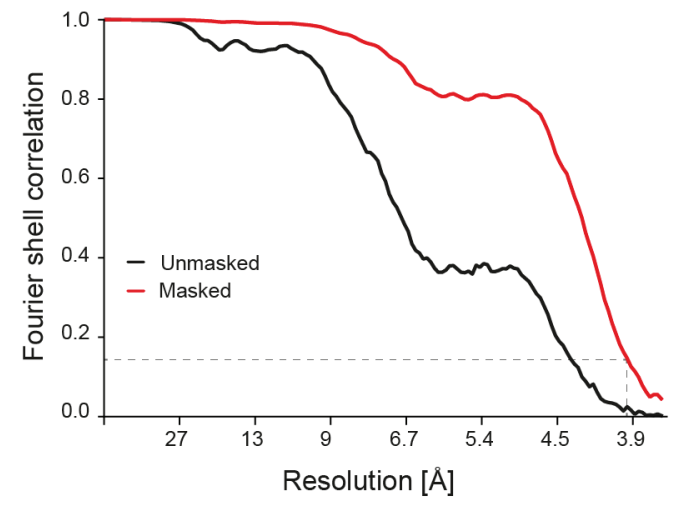

C Local resolution

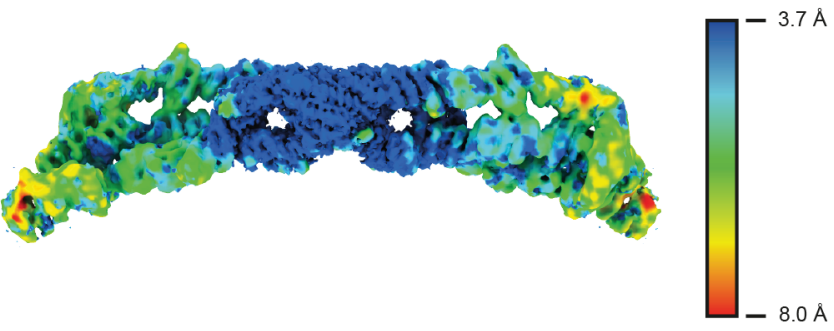

D Angular distribution

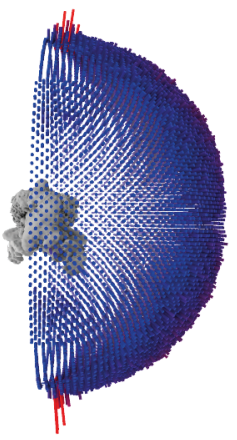




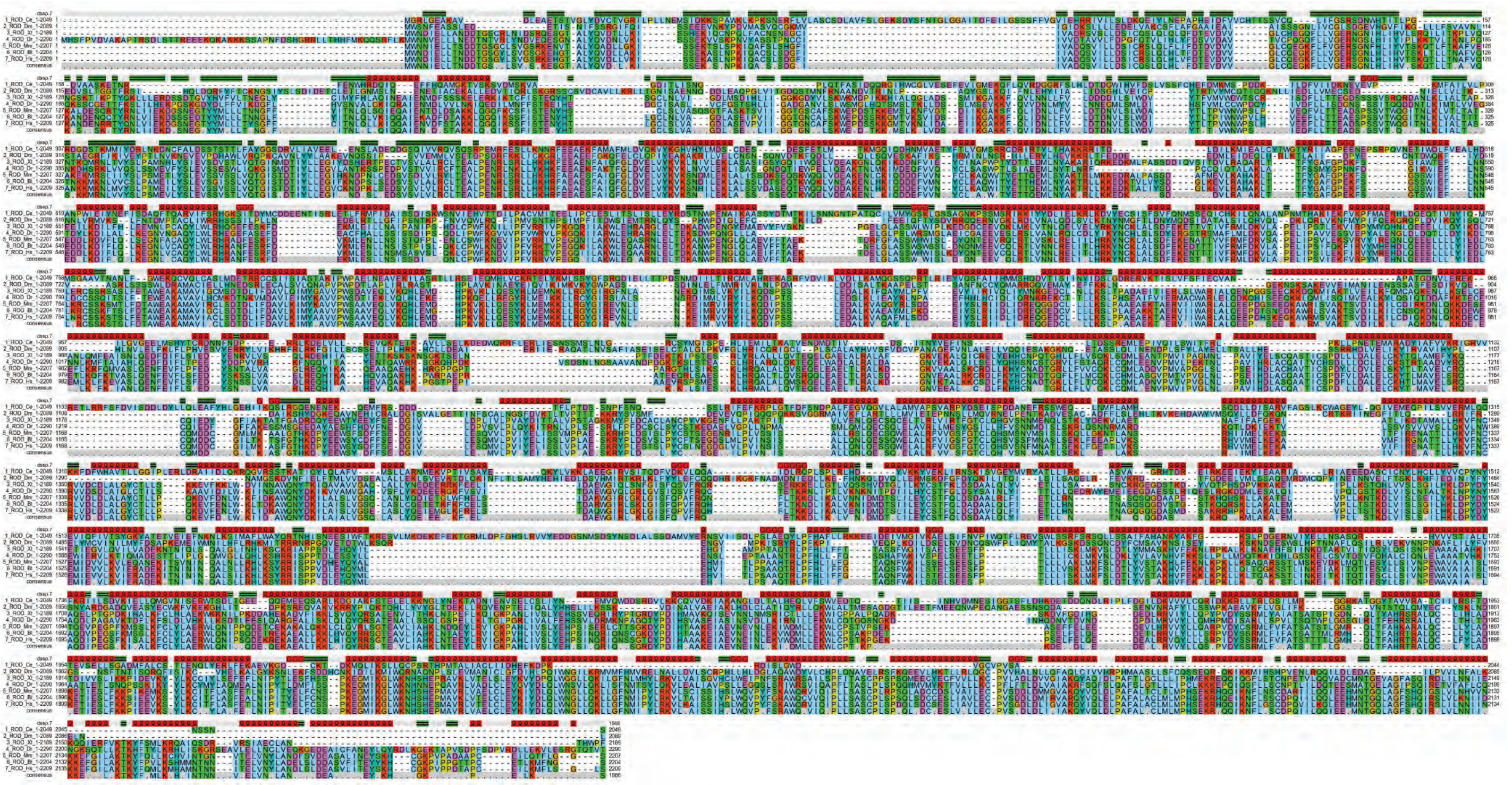

Figure 1 - Supplement 2 


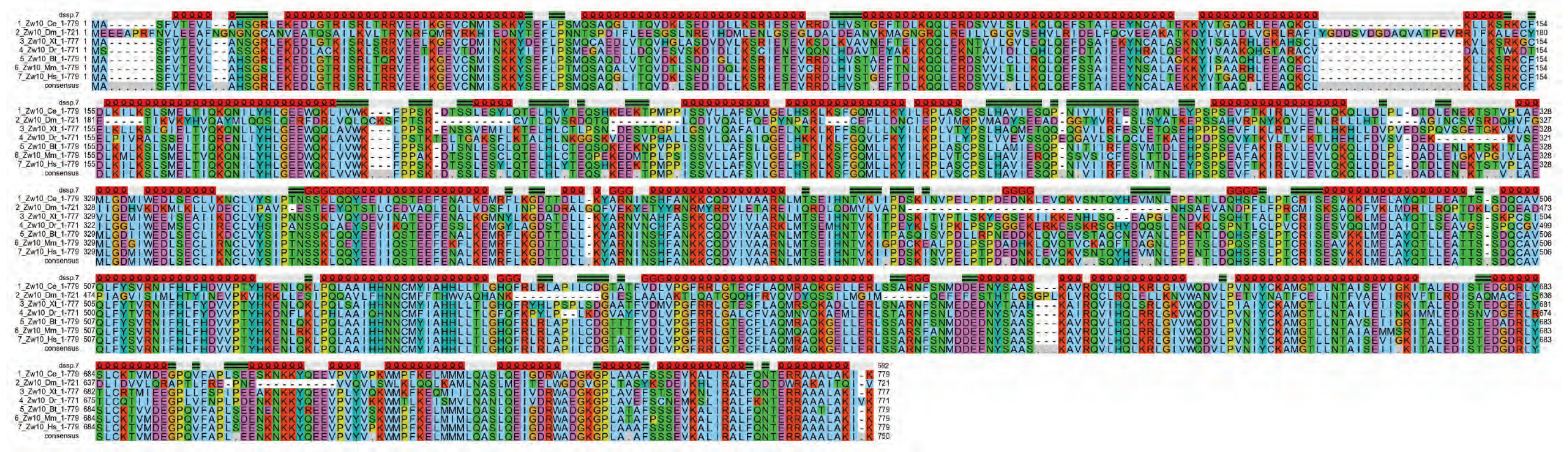

Figure 1 - Supplement 2 


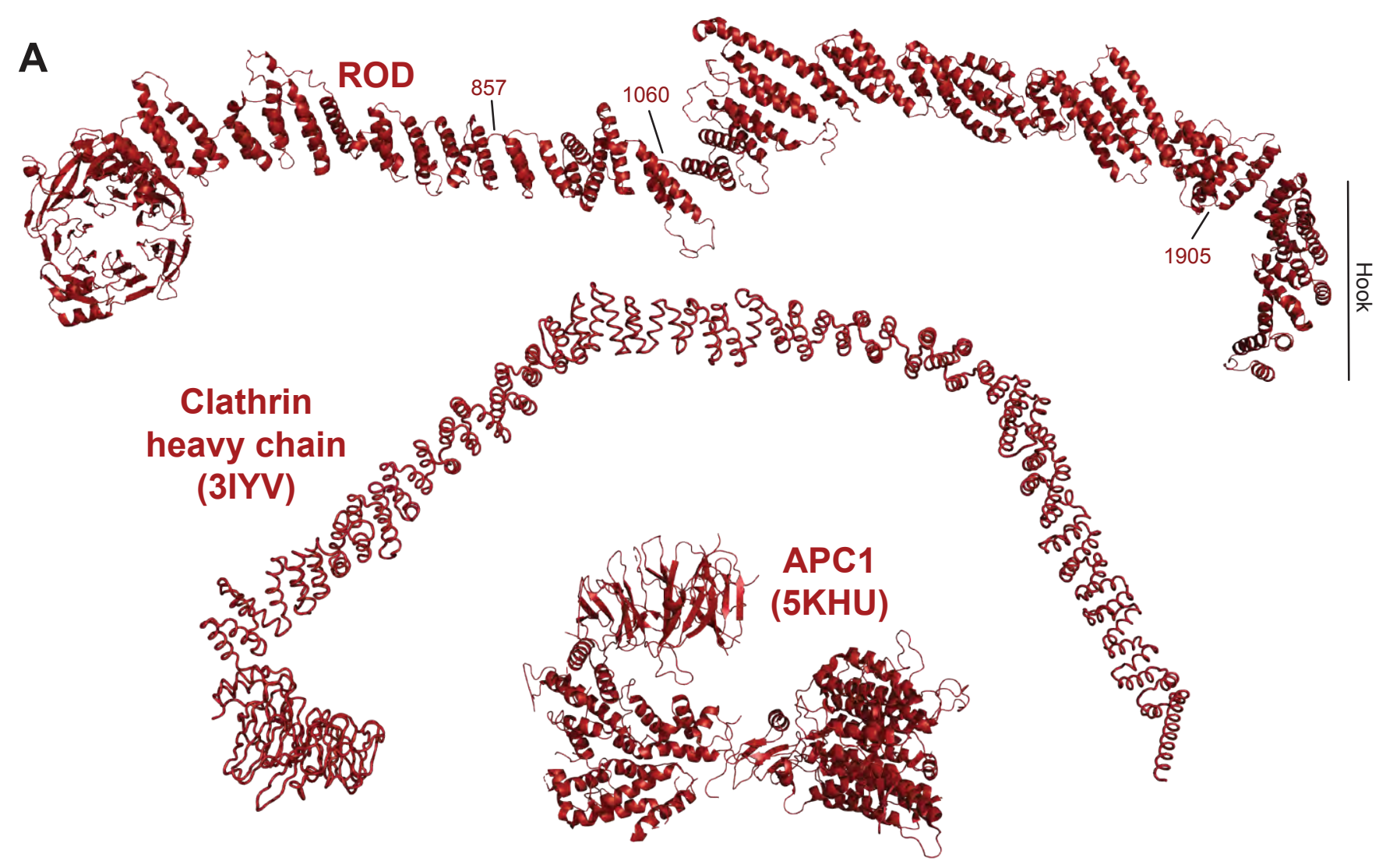

B

ZWILCH

ZWILCH-1 (3IF8)
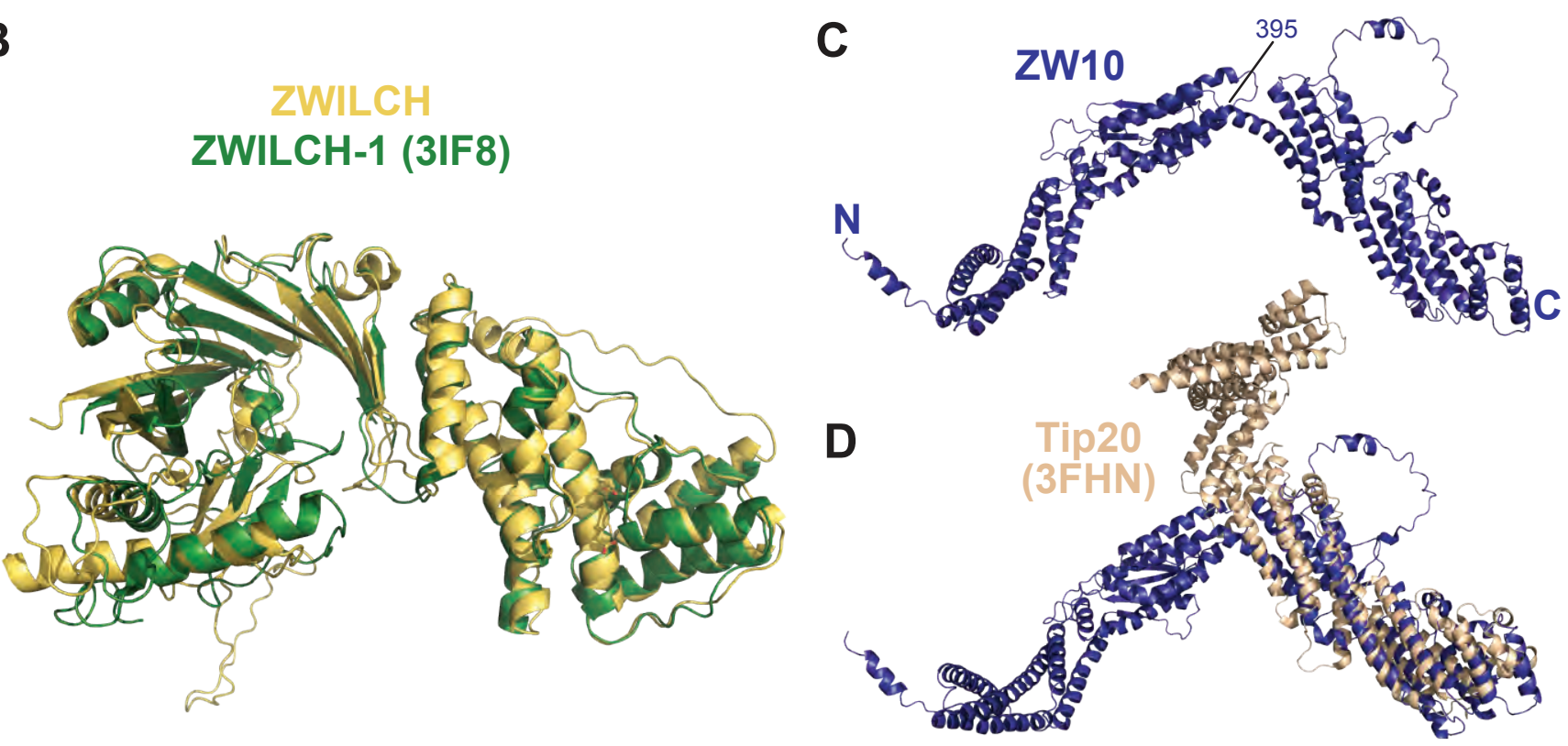

Figure 2 - Supplement 1 
bioRxiv preprint doi: https://doi org/10.1101/2021.12 03.471119; this version posted December 6, 2021. The copyright holder for this preprint (which was not certified by peer review) is the author/funder, who has granted bioRxiv a license to display the preprint in perpetuity. It is made available under aCC-BY-NC 4.0 International license.

A
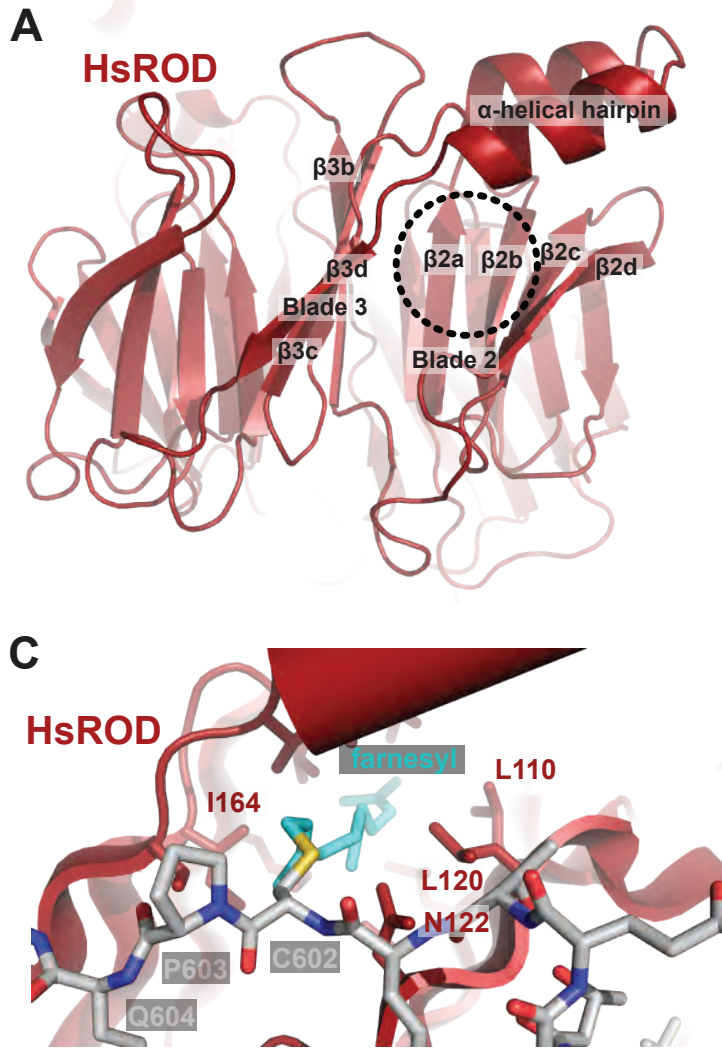

B

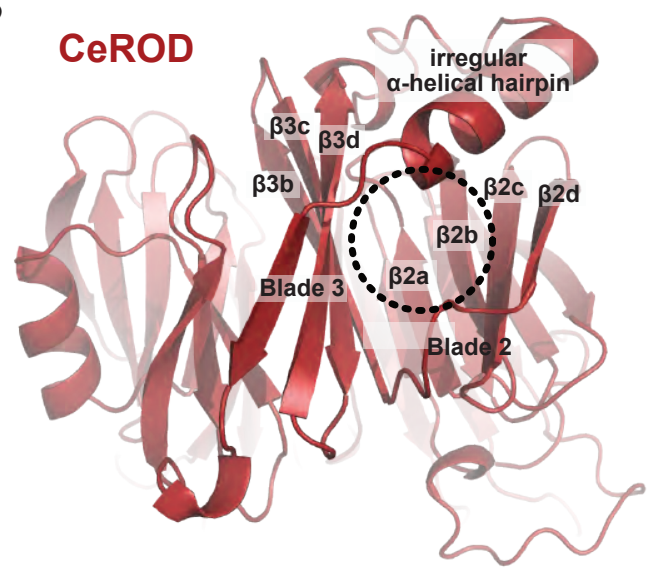

D

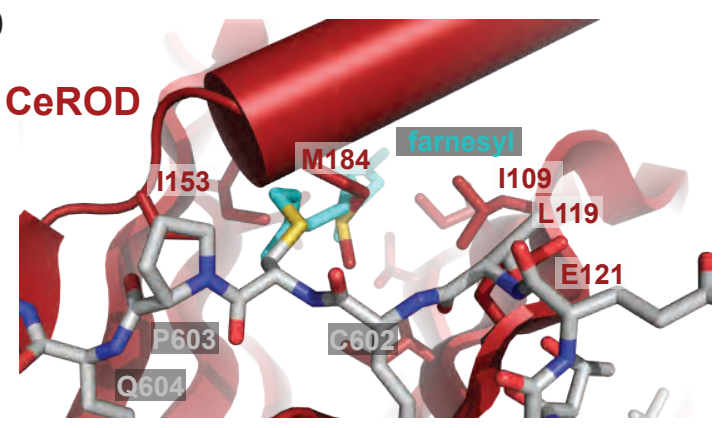

Figure 3 - Supplement 1 
bioRxiv preprint doi: https://doi org/10.1101/2021.12.03.471119; this version posted December 6, 2021. The copyright holder for this preprint (which was not certified by peer review) is the author/funder, who has granted bioRxiv a license to display the preprint in perpetuity. It is made available under aCC-BY-NC 4.0 International license.

A
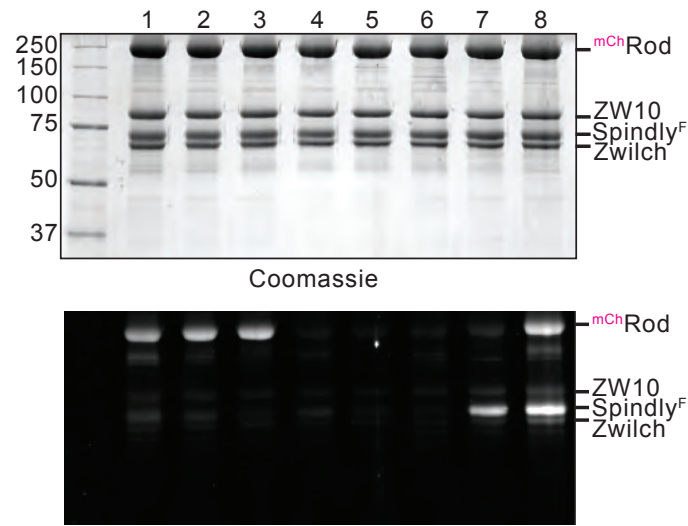

ProQ Diamond

(1) $2 \mu \mathrm{M}$ dephosphorylated ${ }^{\mathrm{mCh}} \mathrm{RZZ}$ and $4 \mu \mathrm{M}$ dephosphorylated Spindly incubated for $15 \mathrm{~h}$ at $20^{\circ} \mathrm{C}$ with $0.5 \mu \mathrm{M}$ MPS1 and ATP

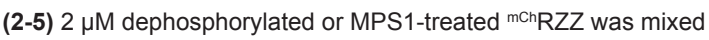
with $4 \mu \mathrm{M}$ dephosphorylated or MPS1-reated Spindly $\mathrm{F}_{\text {in }}$ all

permutations, in presence of MPS1 or $10 \mu \mathrm{M}$ Reversine. The samples were then incubated for $1 \mathrm{~h}$ at $20^{\circ} \mathrm{C}$

(6) $2 \mu \mathrm{M}$ dephosphorylated $\mathrm{mCh}^{\mathrm{R} Z Z}$ and $4 \mu \mathrm{M}$ dephosphorylated Spindly ${ }^{\mathrm{F}}$ incubated $1 \mathrm{~h}$ at $20^{\circ} \mathrm{C}$ with $0.5 \mu \mathrm{M}$ MPS 1 in presence of $10 \mu \mathrm{M}$ Reversine

(7-8) $2 \mu \mathrm{M}$ non-dephosphorylated ${ }^{\mathrm{mCh}} \mathrm{RZZ}$ and $4 \mu \mathrm{M}$ non-dephosphorylated Spindly $\mathrm{F}$ proteins purified from insect cells were incubated ovenight at $20^{\circ} \mathrm{C}$ without (7) or with (8) $0.5 \mu \mathrm{M}$ MPS1 and ATP
B

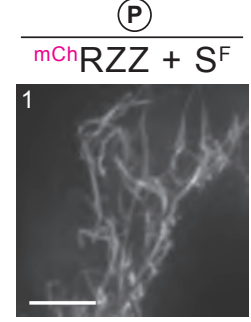

$\stackrel{P}{P}+\stackrel{P}{S F}$

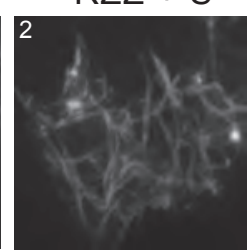

(P)
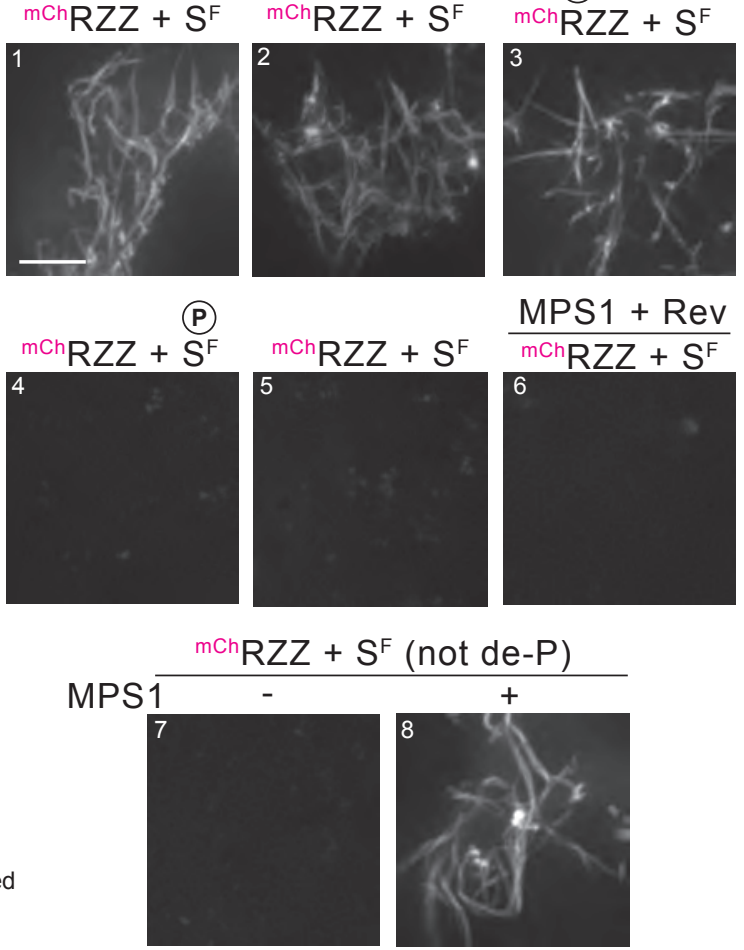

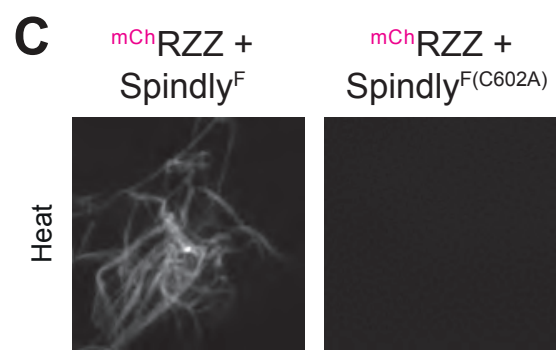

D
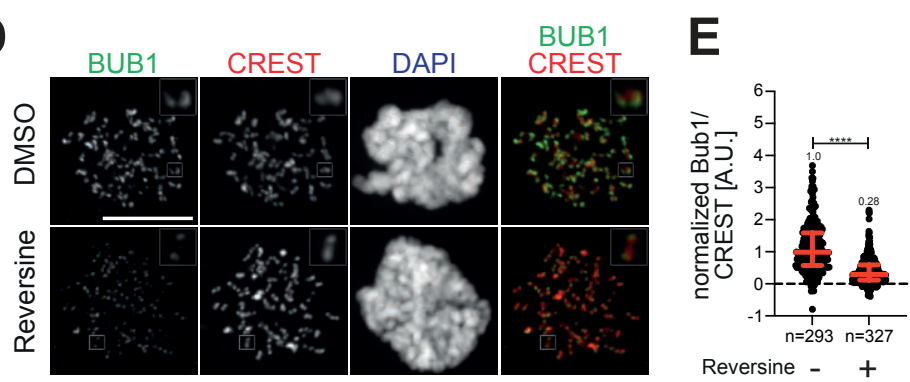

$\mathbf{F}$

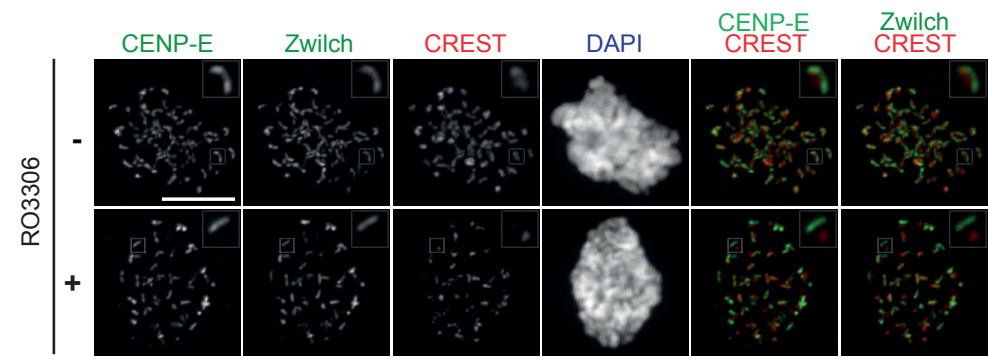

H

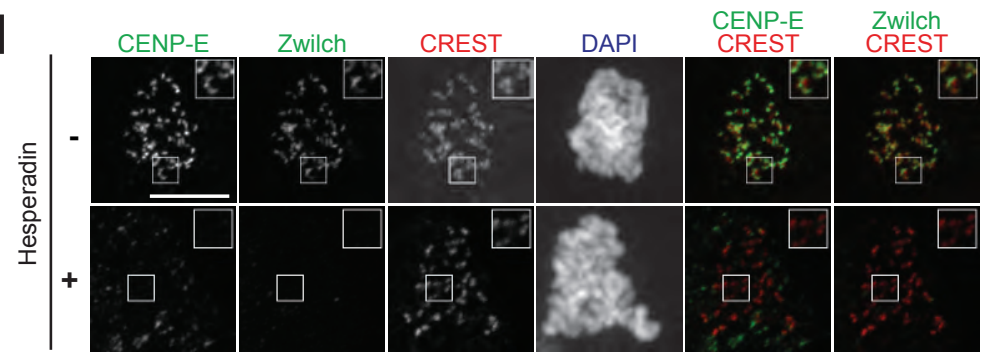

G

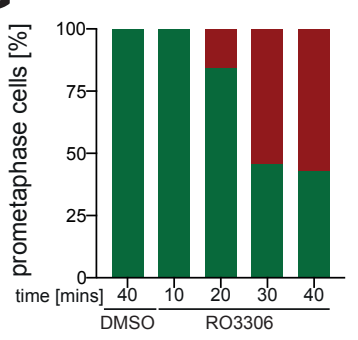

Figure 4 - Figure Supplement 1 
bioRxiv preprint doi: https://doi.org/10.1101/2021.12.03.471119; this version posted December 6, 2021. The copyright holder for this preprint (which was not certified by peer review) is the author/funder, who has granted bioRxiv a license to display the preprint in perpetuity. It is made available under aCC-BY-NC 4.0 International license.

A

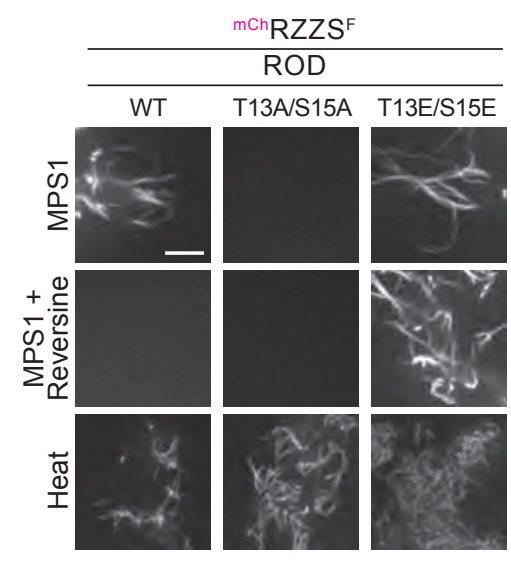

B

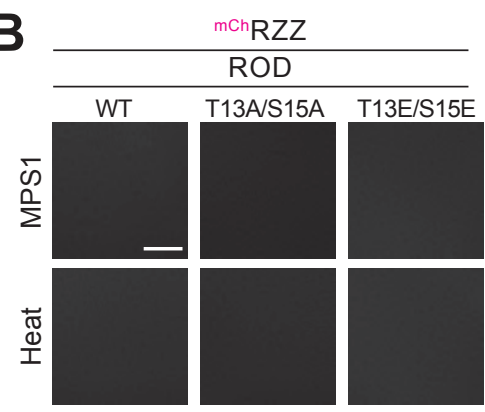

C $\stackrel{乛}{\gtrless}{ }^{35}$ Superose 6 5/150
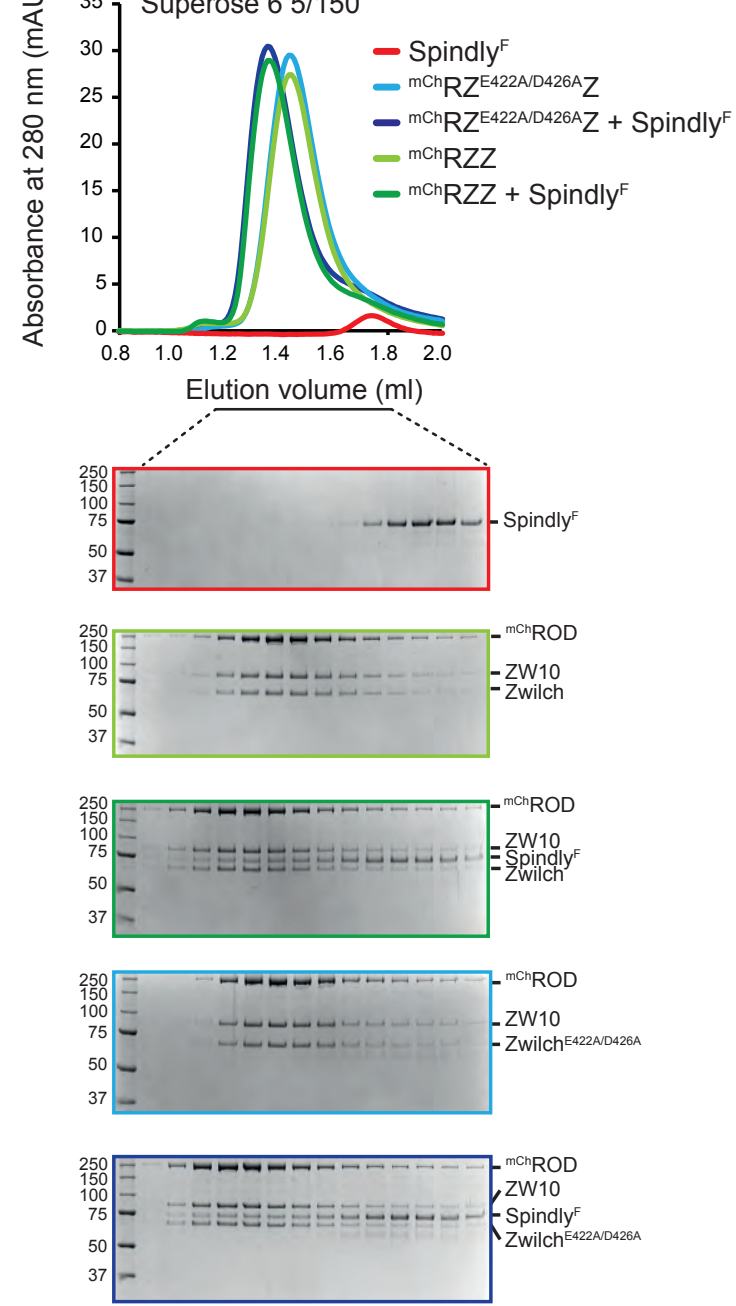

Figure 5 - Supplement 1 
bioRxiv preprint doi: https://doi org/10.1101/2021.12 03.471119; this version posted December 6, 2021. The copyright holder for this

preprint (which was not certified by peer review) is the author/funder, who has granted bioRxiv a license to display the preprint in perpetuity. It is made available under aCC-BY-NC 4.0 International license.

A

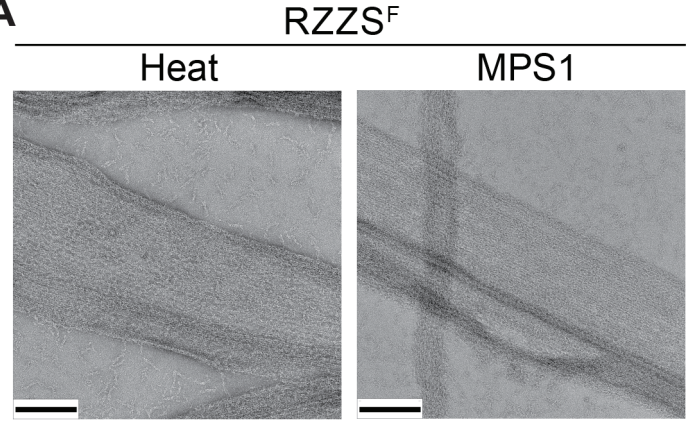

C

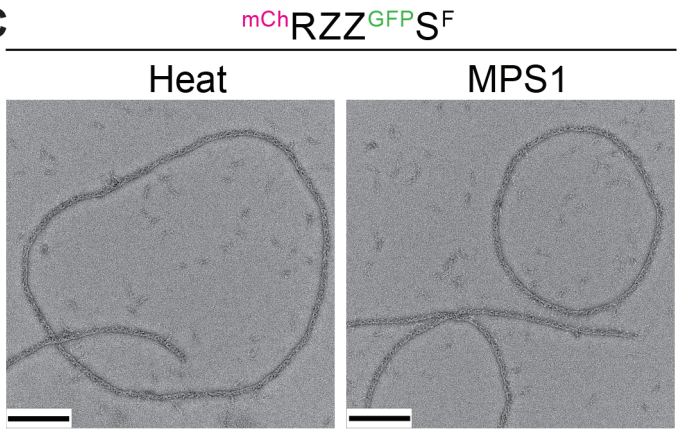

B
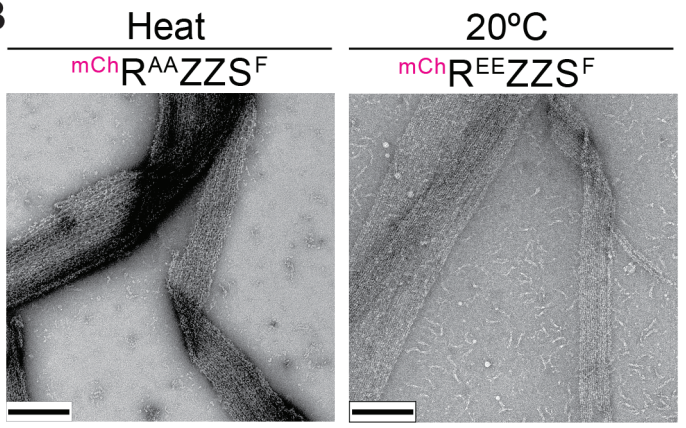

Heat

${ }^{m C h} R^{\triangle 15} Z^{\prime} S^{F}$

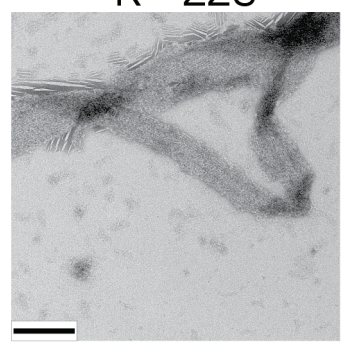

D

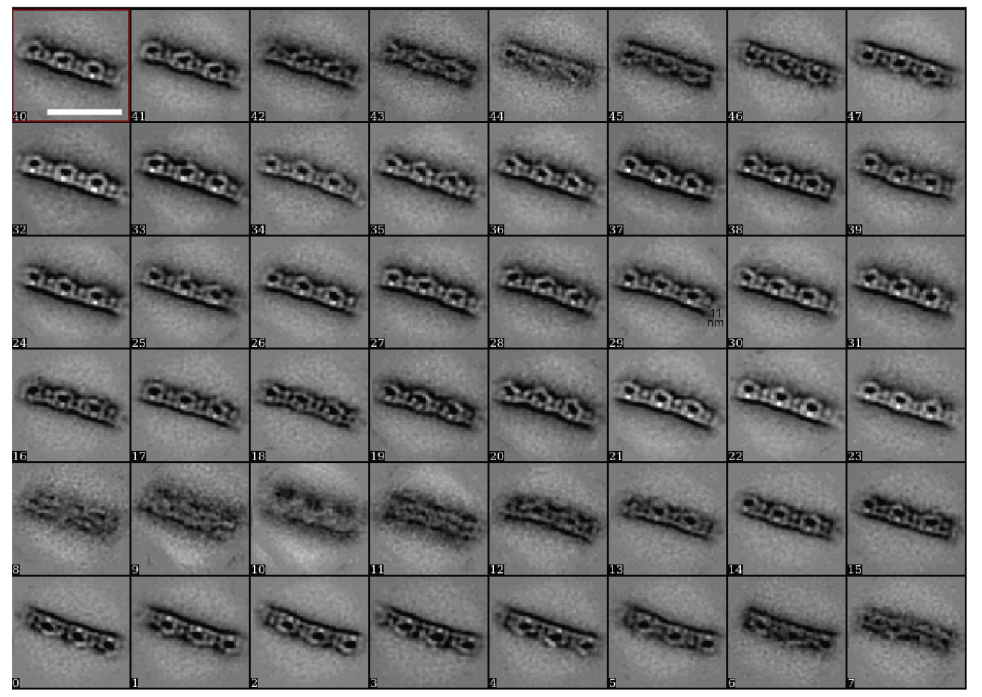

E
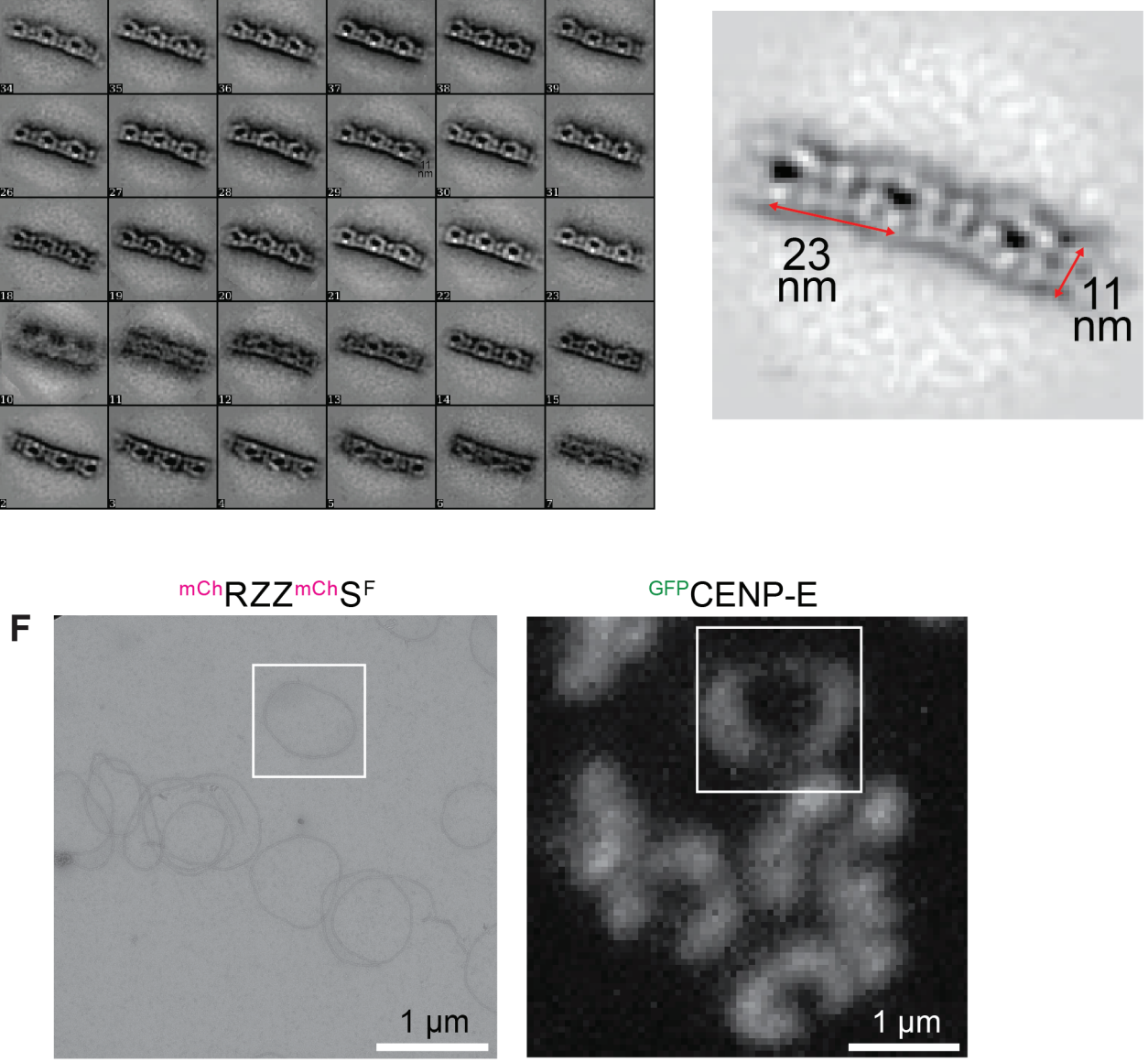

Figure 6 - Supplement 1 
bioRxiv preprint doi: https://doi.org/10.1101/2021.12.03.471119; this version posted December 6, 2021. The copyright holder for this preprint (which was not certified by peer review) is the author/funder, who has granted bioRxiv a license to display the preprint in perpetuity. It is made available under aCC-BY-NC 4.0 International license.

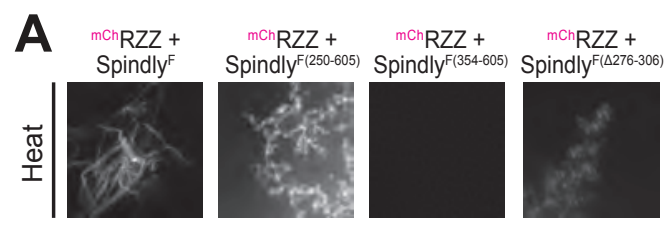

B
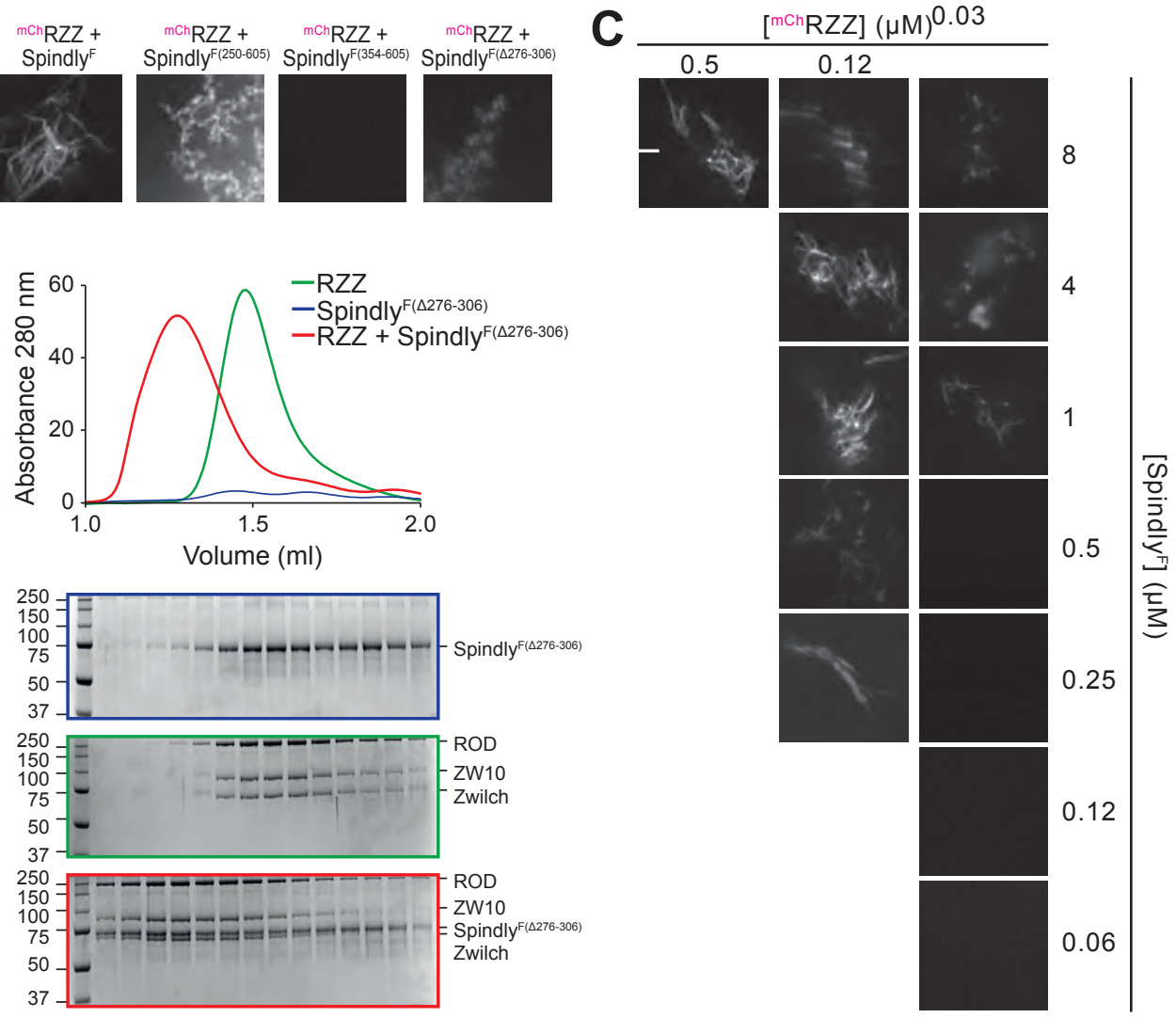

Figure 7 - Supplement 1 